\title{
Discovery of a Cellular Mechanism Regulating Transcriptional Noise
}

3 Ravi V. Desai ${ }^{1,2}$, Maike M.K. Hansen ${ }^{3}$, Benjamin Martin ${ }^{1}$, Chen $\mathrm{Yu}^{4}$, Sheng Ding ${ }^{4,5}$, Matt

4 Thomson $^{6}$, Leor S. Weinberger ${ }^{1,7,8^{*}}$

$6{ }^{1}$ Gladstone|UCSF Center for Cell Circuitry, Gladstone Institutes, San Francisco, CA 94158, USA

$7 \quad{ }^{2}$ Medical Scientist Training Program and Tetrad Graduate Program, University of California, San

8 Francisco, San Francisco, California 94158, USA

$9{ }^{3}$ Institute for Molecules and Materials, Radboud University, Heyendaalseweg 135, 6525 AJ

10 Nijmegen, the Netherlands

$11{ }^{4}$ Gladstone Institute of Cardiovascular Disease, Gladstone Institutes, San Francisco, CA 94158, 12 USA

$13{ }^{5}$ School of Pharmaceutical Sciences, Tsinghua University, Beijing 100084, China

$14{ }^{6}$ Division of Biology and Biological Engineering, California Institute of Technology, Pasadena,

15 California, 91125, USA

167 Department of Pharmaceutical Chemistry, University of California, San Francisco, San

17 Francisco, CA 94158, USA

$18{ }^{8}$ Department of Biochemistry and Biophysics, University of California, San Francisco, San

19 Francisco, CA 94158, USA

$20 *$ Correspondence to: leor.weinberger@gladstone.ucsf.edu 


\section{Abstract}

22 Stochastic fluctuations in gene expression ('noise') are often considered detrimental but, in

23 other fields, fluctuations are harnessed for benefit (e.g., 'dither' or amplification of thermal

24 fluctuations to accelerate chemical reactions). Here, we find that DNA base-excision repair

25 amplifies transcriptional noise, generating increased cellular plasticity and facilitating

26 reprogramming. The DNA-repair protein Apex1 recognizes modified nucleoside substrates

27 to amplify expression noise-while homeostatically maintaining mean levels of expression-

28 for virtually all genes across the transcriptome. This noise amplification occurs for both

29 naturally occurring base modifications and unnatural base analogs. Single-molecule

30 imaging shows amplified noise originates from shorter, but more intense, transcriptional

31 bursts that occur via increased DNA supercoiling which first impedes and then accelerates

32 transcription, thereby maintaining mean levels. Strikingly, homeostatic noise amplification

33 potentiates fate-conversion signals during cellular reprogramming. These data suggest a

34 functional role for the observed occurrence of modified bases within DNA in embryonic

35 development and disease. 
Main Text

37 From Brownian motion to electrical 'shot' noise, fluctuations are fundamental to physical

38 processes. Since the 1800 s (1), fluctuations have been recognized to dynamically shape the

39 distribution of microstates a system adopts, and modulation of fluctuations has been harnessed

40 throughout engineering and the sciences. For example, in chemistry, thermal fluctuations-

41 amplified via temperature increase (e.g., Bunsen Burners) - accelerate reactions (2); in

42 engineering, amplification of electrical, acoustic, or mechanical fluctuations (i.e., 'dither', from

43 the Middle English "didderen" meaning to "tremble") is used for signal recovery (3), and in

44 neuroscience, electrophysiological fluctuations-first reported in the 1950s (4) — are clinically

45 amplified to improve sensorimotor function (5-7). Such 'dither' approaches break Poisson dependency so that $\Delta$ variance $\neq \Delta$ mean.

48 Evolutionary theories dating to the 1960s (8-10) proposed that biological organisms maximized 49 fitness by harnessing putative fluctuations to enable probabilistic 'bet-hedging' decisions.

50 Subsequent studies showed that intrinsic molecular fluctuations in gene expression (i.e., stochastic

51 'noise'), modulated by gene-regulatory circuits, enabled probabilistic fate selection (Fig. 1A) in 52 diverse biological systems (11-13). Open questions remain as to whether cellular noise control is

53 limited to inherently locus-specific gene-regulatory circuits or if generalized noise-modulation

54 mechanisms exist, if and how such mechanisms might orthogonally tune noise independent of mean, and, given the detrimental effects of noise, if such putative mechanisms might be regulated 'on-demand' to potentiate cell-fate specification.

58 Non-genetic variability or noise in gene expression, often quantified by measurement of cell-tocell variability in reporter expression, can arise from multiple sources, both intrinsic and extrinsic.

60 In mammalian cells, intrinsic noise originates from episodic transcriptional 'bursts' (14-17) 61 initiated by promoter toggling between $\mathrm{ON}$ and OFF states (Fig. 1B). The two-state random-

62 telegraph model describes this bursting via two parameters: (i) the fraction of time a promoter is 63 active $\left(K_{O N} /\left[K_{O N}+K_{O F F}\right]\right)$, and (ii) the number of transcripts produced during the ON state (burst 64 size, $\left.K_{T X} / K_{O F F}\right)(18-20)$. These bursting parameters are tuned by regulatory machinery (21) like 65 histone acetyltransferases, which can increase burst frequency by facilitating nucleosome 66 clearance from promoters thereby increasing mean transcriptional levels (22). Increases in mean 
67 expression $(\mu)$ are typically accompanied by a stereotypical reduction in noise measured by

68 coefficient of variation, $\mathrm{CV},(\sigma / \mu)$ (Fig. 1B), whereas stressors that decrease mean are typically

69 accompanied by an increase in noise (23-25). This $1 / \mu$ scaling of noise can be broken by gene-

70 regulatory circuits such as feedback and feedforward loops (26), and some small-molecule

71 pharmaceuticals can modulate transcriptional fluctuations/noise $(\sigma / \mu)$ independent of change in

72 mean $(\mu)(27,28)$. Since some molecules can amplify expression noise of diverse unrelated

73 promoters $(27,29)$, we asked if these molecules might be functioning via disruption or

74 enhancement of a putative cellular noise-control mechanism.

A series of screens (Fig. S1) identified one compound, 5'-iodo-2'-deoxyuridine (IdU), which consistently increased expression noise of multiple transcriptional reporter constructs in diverse cell types. To test the generality of this noise amplification effect we focused on mouse embryonic stem cells (mESCs), due to extensive characterization of their developmental and transcriptional

80 characteristics (30-34). Strikingly, single-cell RNA sequencing (scRNA-seq) of mESCs

81 maintained in 2i/LIF media — after filtering and normalization using Seurat (35) — showed that IdU

82 amplified cell-to-cell variability in transcript levels (i.e., transcript noise) for virtually all genes 83 across the genome-4,578 genes analyzed (Fig. 1C) - with little alteration in mean transcript 84 levels for most genes, as analyzed by either $\mathrm{CV}^{2}\left(\sigma^{2} / \mu^{2}\right)$ or variance $\left(\sigma^{2}\right)$ versus mean (Figs. 1C85 D). To account for the Poisson scaling of variance on mean, transcript noise was also quantified using the Fano factor $\left(\sigma^{2} / \mu\right)$, which measures how noise deviates from Poisson scaling $\left(\sigma^{2} / \mu=1\right)$ (20, 36, 37). Despite mean-expression levels exhibiting minimal changes (Fig. 1E), the Fano factor increased for $>90 \%$ of genes (Fig. 1F) with lowly expressed genes showing a slightly greater change in Fano (Fig. S2). These results of a global increase in transcript noise with little change in mean levels are in stark contrast to the effects of transcriptional activators or cellular stressors that alter noise in a stereotypic manner together with changes in mean $(23,38)$.

To account for technical noise and quantify statistical significance of changes in noise and mean,

94 we used an established Bayesian hierarchical model $(39,40)$ to create probabilistic, gene-specific 95 estimates of both mean expression and cell-to-cell transcript variability. Of the 4,578 genes, the 96 algorithm classified 945 genes $(\sim 20 \%)$ as highly variable, whereas 113 genes ( 2\%) showed a 97 significant change in mean expression (Figs. 1G-H). Bulk RNA-seq measurements of mean 
abundances-performed using ERCC spike-ins for normalization-confirmed the scRNA-seq

99 findings (Fig. S3). Thus, analyses from two methods (Seurat and BASiCS) show that IdU induces

100 a significant increase in transcript variability (expression noise) but comparatively little change in

101 mean expression in mESCs.

103 To examine if certain characteristics could explain a gene's potential for noise enhancement we 104 examined (i) gene length, (ii) promoter and (iii) gene-body AT content, (iv) number of exons, (v)

105 TATA-box inclusion and (vi) strand orientation. None of these characteristics exhibited predictive 106 power or correlated with a gene's potential for noise enhancement (Fig. S4). However, genes 107 susceptible to high noise enhancement were preferentially located within the interior of 108 topologically associated domains (TADs), suggesting gene topology influences potency of noise 109 enhancement (Fig. S4). Ontology analysis of highly variable genes showed enrichment of house110 keeping pathways along with pluripotency maintenance factors, particularly Sox2, Oct4, Nanog 111 and Klf4 (Figs. 1H, S5). As these pluripotency maintenance factors are key influencers of cell112 fate specification, we next focused on the molecular mechanisms driving their amplified transcript 113 noise.

115 We first tested whether the enhanced variability arose from extrinsic factors, which include cell116 cycle phase and cell-type identity $(16,41-44)$. Cells within the scRNA-seq dataset were 117 computationally assigned a cycle stage (G1, S, G2/M) (45) which showed that Nanog, Oct4, Sox2 118 and Klf4 were highly variable in each cell-cycle phase, indicating that their variability is not cell119 cycle dependent (Fig. S6). Moreover, pseudo-time analysis showed no bifurcations, indicating 120 transcriptional variability was not due to a differentiation-induced mixture of cell-types (Fig. S7).

122 Extrinsic variability may also arise from the coordinated propagation of noise through gene123 regulatory networks $(46,47)$ and can be measured by gene-to-gene correlation matrices $(48,49)$.

124 If the increase in global transcript noise is extrinsic, expression correlation between network 125 partners would increase or remain unchanged. Analysis of gene-to-gene correlation matrices 126 showed that $\sim 80 \%$ of gene-gene pairs lost correlation strength following IdU treatment (Figs. 2A 127 and S8), indicating that enhanced expression noise is uncorrelated and not consistent with an 128 extrinsic noise source. Exclusion of these extrinsic noise sources suggested that IdU amplifies 
129 intrinsic noise arising from stochastic fluctuations in transcript birth (promoter toggling) or death

130 (degradation).

132 To test whether a change in promoter toggling could account for IdU-enhanced noise, we used 133 single-molecule RNA FISH (smRNA-FISH) to count both nascent and mature transcripts of 134 Nanog, a master regulator of pluripotency. Spot counting was performed on a mESC line in which 135 both endogenous alleles of Nanog are fused to eGFP. This fusion does not alter mRNA or protein 136 half-life or impair differentiation potential (50). To target mature transcripts, smRNA-FISH 137 probes to eGFP were used, and to minimize extrinsic noise, analyses were limited to cells of similar 138 sizes (Fig. S9A). Consistent with scRNA-seq, smRNA-FISH showed a large increase in cell-to139 cell variability of mature Nanog transcripts ( 2 -fold increase in Fano) with little change in mean 140 Nanog levels (Fig. 2B). Fewer IdU-treated cells exhibited active transcriptional centers (TCs), but 141 the number of nascent mRNAs at each TC increased (Figs. 2C-D). Fitting of the two-state 142 random-telegraph model to smRNA-FISH data revealed that increased variability was due to a 143 shortened burst duration (increased $k_{O F F}$ ) and amplified transcription rate (higher $\left.k_{T X}\right)$ (Fig. S9B, 144 Table S2). These results represent direct validation of previous predictions $(20,27)$ that enhanced 145 noise could arise from reciprocal changes in transcriptional burst duration $\left(1 / k_{O F F}\right)$ and intensity $146\left(k_{T X}\right)$.

148 To test if enhanced transcript variability transmitted to the protein level, we performed flowcytometric analysis of Nanog-GFP reporter protein. In IdU-treated cells, the Nanog protein Fano 150 factor increased by $\sim 3$-fold, with little change in mean, indicating that mRNA variability from 151 altered promoter toggling indeed resulted in changes to protein noise (Fig. 2E). The increase in 152 protein noise showed no dependency on cell-cycle (Fig. S10C-D) despite G1-to-S cell-cycle 153 progression being slightly slowed by IdU treatment (Fig. S10A-B). Consistent with the extrinsic 154 noise analysis above, there was no evidence of aneuploidy following IdU treatment (Fig. S10A), 155 precluding the possibility that increased noise results from a sub-population of cells with non156 physiologic gene-copy numbers.

158 Given that Nanog noise was intrinsic and transmitted to the protein level, we next tested a previous 159 theoretical prediction about Nanog. When cultured in 2i/LIF, mESCs exhibit Nanog protein 
160 expression that is unimodal and high, but when cultured in serum/LIF, mESCs exhibit bimodal 161 Nanog expression with both a low Nanog state and a high Nanog state (Fig. S11) (34). Theories

162 predicted that increased transcriptional noise would drive greater excursions from the high Nanog 163 state into the low Nanog state (51). We found that in mESCs cultured in serum/LIF, IdU-induced 164 amplification of Nanog noise did indeed generate greater excursions into the low Nanog state (Fig. S11), verifying theoretical predictions. This result demonstrates how promoter toggling can drive 166 Nanog state-switching thereby altering differentiation potential.

To verify that enhanced noise is not a population-level phenomenon brought on by differential 169 responses to IdU in distinct cellular subpopulations (i.e., verify 'ergodicity' and that individual 170 cells exhibit increased fluctuations), we used live-cell time-lapse imaging to quantify both the 171 magnitude (intrinsic- $\mathrm{CV}^{2}$ ) and frequency content (1/half-autocorrelation time) of Nanog

172 fluctuations. Single-cell tracking of individual cells showed that IdU induced a 2-fold increase in 173 the magnitude (intrinsic-CV $\mathrm{CV}^{2}$ ) of fluctuations (Figs. 2F, S12A), and auto-correlation analysis of 174 detrended trajectories showed a broadening of the frequency distribution to higher spectra, 175 indicating reduced memory of protein state (Fig. S12B). These higher frequency fluctuations are 176 consistent with amplification of a non-genetic, intrinsic source of noise $(52,53)$ because genetic 177 sources of cellular heterogeneity, such as promoter mutations, would lead to longer retention of 178 protein states (increased memory) (54). In silico sorting of cells based on starting Nanog 179 expression verified that noise enhancement was not dependent on memory of initial state (Fig. $180 \mathrm{~S} 13)$. Fluctuations in promoter toggling therefore drive individual cells to dynamically explore a 181 larger state-space of Nanog expression. To further validate that IdU perturbs an intrinsic source 182 of noise, we used a mESC line in which the two endogenous alleles of Sox 2 are tagged with P2A183 mClover and P2A-tdTomato, respectively, to enable quantification of the intrinsic and extrinsic 184 components of noise. Treatment with IdU increased Sox 2 intrinsic noise greater than 2-fold across 185 all expression levels (Fig. S14) further validating that IdU enhances intrinsic noise.

187 To test if a generalized stress response could explain the noise enhancement induced by IdU, we 188 subjected Nanog-GFP mESCs to UV radiation for 15, 30, or 60 minutes. Both the mean and Fano 189 factor of Nanog expression decreased for all timepoints, which markedly differs from IdU 
190 treatment (Fig. S15). This result further indicates that IdU does not perturb an extrinsic or global

191 noise source; rather, it perturbs an intrinsic source of noise (i.e., promoter toggling).

193 To pinpoint the molecular mechanism, 14 additional nucleoside analogs (Table S3) were screened 194 for noise enhancement effects. 5'-bromo-2'-deoxyuridine (BrdU), 5-hydroxymethylcytosine $195 \mathrm{(hmC}$ ), and 5-hydroxymethyluridine (hmU) also increased Nanog Fano factor to varying degrees 196 (Fig. 3A). Intriguingly, hmU and $\mathrm{hmC}$ are naturally produced by the Ten Eleven Translocation 197 (Tet) family of enzymes during oxidation of thymine and methylated cytosine respectively (55198 58). Given that these base modifications are removed via base-excision repair (BER), we surmised 199 that their incorporation and removal from genomic DNA may be responsible for noise 200 enhancement (Fig. 3B) (59, 60). To test this, we knocked down 25 genes (3 gRNAs/gene, Table 201 S4) involved in nucleoside metabolism and DNA repair using CRISPRi, and quantified how these 202 knockdowns affected IdU's noise enhancement. We identified 2 genes: AP Endonuclease 1 203 (Apex1) and thymidine kinase 1 (Tk1) whose knockdown abrogated noise enhancement (Fig. 3C). 204 Knockdown was confirmed via RT-qPCR (Fig. S16).

Tk1 adds a requisite gamma-phosphate group to diphosphate nucleotides prior to genomic incorporation (Fig. 3B) (61). Our knockdown results indicated that phosphorylation of IdU by Tk1 and subsequent incorporation of phosphorylated IdU into the genome may be necessary for noise enhancement. To validate this, we tested the effect of treatment with $10 \mu \mathrm{M}$ IdU combined 211 returned Nanog noise to baseline levels (Fig. S17), indicating that noise enhancement is dependent 212 on IdU incorporation. The reduction in Nanog noise with addition of exogenous thymidine also 213 suggests that IdU-induced noise amplification is not a generic effect of nucleotide imbalances 214 within the cell.

216 Apex1 (a.k.a., Ref-1, Ape1) plays a pivotal role in the BER pathway as it incises DNA at 217 apurinic/apyrimidinic sites via an endonuclease domain, allowing for subsequent removal of the 218 sugar backbone and patching of the gap $(62,63)$. To confirm the knockdown results, we attempted 219 to knockout Apex1 in mESCs. However, the knockout was lethal, in agreement with previous reports (64). As an alternative, we used a small-molecule inhibitor (CRT0044876) specific for the 
221 Apex1 endonuclease domain (65). Unexpectedly, the combination of CRT0044876 with IdU 222 synergistically increased Nanog protein variability, without significantly changing the mean (Fig.

223 3D).

224

225 The contrasting effects of Apex1 knockdown and catalytic inhibition implied that a physical rather 226 than enzymatic quality of the protein is responsible for modulation of transcriptional bursting. In

227 support of this, Apex1 induces helical distortions and local supercoiling to identify mismatched 228 bases $(66,67)$. Furthermore, catalytically inactive Apex1 binds DNA with higher affinity (68).

229 Therefore, CRT0044876 may lengthen Apex1 residence times on DNA, thus synergistically 230 amplifying topological reformations. Taken together with evidence that supercoiling sets 231 mechanical bounds on transcriptional bursting (69-71), we next asked whether Apex1 recruitment 232 impacts supercoiling levels.

234 To assay supercoiling, we used a psoralen-crosslinking assay in which mESCs are incubated with 235 biotinylated-trimethylpsoralen (bTMP), which preferentially intercalates into negatively 236 supercoiled DNA $(72,73)$. To eliminate DNA replication as a contributor of supercoiling, 237 aphidicolin is added to inhibit DNA polymerases prior to bTMP incubation (74). IdU treatment 238 significantly increased genomic supercoiling as demonstrated by a $\sim 2$-fold increase in bTMP 239 intercalation (Fig. 3E). The combination of IdU and CRT0044876 further increased intercalation, 240 suggesting that supercoiling levels are correlated with noise enhancement through increased 241 Apex1-DNA interactions (Fig. 3E). IdU treatment followed by a short incubation with bleomycin 242 (which decreases supercoiling through double-stranded breaks) reduced bTMP intercalation below 243 the DMSO control level, indicating IdU alone in uncoiled DNA does not increase intercalation 244 (Fig. S18).

246 If DNA topology influences transcriptional bursting, additional modifiers of supercoiling should 247 also affect Nanog noise. Topoisomerase 1 and 2a (Top1 and Top2a, respectively) relax coiled 248 DNA through the introduction of single- and double-stranded breaks, respectively. Knockdown 249 of Top1 and Top2a via CRISPRi increased Nanog protein variability (Fig. 3F). Furthermore, 250 inhibition of topoisomerase activity with the small-molecule inhibitors topotecan and etoposide 251 recapitulated these effects (Fig. S19). Taken together with psoralen-crosslinking data, these results 
suggest that Apex1-induced supercoiling tunes gene-expression fluctuations without altering mean expression levels.

To understand the mechanism by which Apex1 might increase transcriptional noise without altering mean expression levels, we developed a series of minimalist computational models to account for the experimental data (supplementary text 2). We ran Monte Carlo simulations of each model using smRNA-FISH data for parameterization (Table S2); this revealed that a transcriptioncoupled-repair (TCR) mechanism best accounts for the noise-without-mean amplification (Figs. 4A-B, S20-21, supplementary text 5). Importantly, Apex1 binding triggers a transcriptionally nonproductive, negatively supercoiled state, whereas unbinding of Apex 1 allows mRNA production to resume with an amplified transcription rate. This amplification may originate from the increased negative supercoiling during repair which can facilitate upstream binding of transcriptional machinery in preparation for when repair is complete (75-82). The ability to render a gene transcriptionally non-productive while also stimulating recruitment of transcriptional resources points to a homeostatic mechanism: the BER pathway maintains gene-expression homeostasis

267 (i.e., mean) by amplifying transcriptional fluctuations through reciprocal modulation of burst 268 intensity and duration (Fig. 4B).

Sensitivity analysis of the Apex1 TCR model revealed that orthogonal modulation of Nanog mean and noise is possible within a large portion of the parameter space (Fig. S22A-B, supplementary text 6). As validation, we tested the effect of 96 concentration combinations (Table S6) of IdU

273 and CRT0044876 to perturb the rates of Apex1 binding and unbinding respectively. The 274 experimental results confirmed model predictions, showing that Nanog noise could be tuned 275 independently of the mean (Fig. 4C). Testing of BrdU and hmU further validated that parameter 276 regimes exist where noise can be regulated independent of mean (Fig. S23). The hmU data in 277 particular showed that the BER pathway can amplify noise while maintaining mean expression 278 when removing a naturally occurring base modification. Additionally, sensitivity analysis 279 indicated that for genes whose $K_{O F F} \gg K_{O N}$ (i.e., lowly expressed genes), IdU treatment would 280 increase mean abundance (Fig 22E). This prediction was verified experimentally with bulk RNA281 seq measurements of transcript abundance in mESCs treated with IdU (Fig S3), as all 98 of the up282 regulated genes reside within the lowest expression regime. 
284 To test whether the homeostatic mechanism of BER applies to additional genes, mRNA

285 distributions from the scRNA-seq dataset were fit to a Poisson-beta model (two-state model)

286 allowing for estimation of $K_{O N}, K_{O F F}$, and $K_{T X}(83,84)$. A consistent pattern emerged for genes

287 classified as highly variable: 80\% exhibited increased rates of promoter inactivation $\left(K_{O F F}\right)$ and

$28884 \%$ had increased transcription rates $\left(K_{T X}\right)$ (Fig. S24). Alignment of these rate estimates with

289 predictions from the TCR model revealed that the BER pathway can alter noise while maintaining

290 transcriptional homeostasis by individually tailoring expression fluctuations for genes with vastly

291 different bursting kinetics (Fig. S25, supplementary text 7).

293 We next asked if this amplification of transcriptional variability acted to enhance cellular plasticity

294 as previously suggested (85). Using a neural-network approach, we reconstructed the Waddington

295 landscape based on a predictive model of gene-gene interactions inferred from the scRNA-seq data

296 (86). In this approach, each cell has a characteristic energy determined by its proximity to an

297 attractor state, with lower energy values corresponding to greater stability. The analysis indicated

298 that cells exposed to IdU lie at a higher altitude on this landscape, indicating destabilization of

299 cellular identity and greater developmental plasticity (Fig. S26). Numerical simulations of the TCR

300 model then verified that IdU-mediated amplification of transcriptional noise has the potential to

301 increase responsiveness to activation stimuli (Fig. 4D). The complementary abilities of IdU-

302 mediated noise amplification to destabilize cellular identity and potentiate responsiveness to fate

303 signals suggested that IdU might facilitate cellular reprogramming.

To experimentally verify these predictions, we tested if IdU could potentiate conversion of differentiated cells into pluripotent stem cells using two cellular reprogramming systems. The first assay utilized mouse embryonic fibroblasts (MEFs) that express GFP from the endogenous Nanog locus and harbor stably integrated, doxycycline-inducible cassettes for three of the Yamanaka factors: Oct4, Sox2, and Klf4 (OSK). As confirmation that IdU acts as a noise-enhancer in this system, treatment of secondary MEFs with IdU for 48 hours in standard MEF media caused

311 increased variability in Nanog protein expression (Fig. S27A) with no changes in cell-cycle 312 progression (Fig. S27B). Strikingly, IdU supplementation for the first 48 hours of a 10-day 313 reprogramming course enhanced the formation of pluripotent colonies as measured by alkaline 
314 phosphatase staining (Fig. 4E). Bulk RNA-seq at days 2 and 5 of reprogramming (Fig. S27C) and 315 flow-cytometric analysis at day 10 (Fig. 4F) demonstrate that early-stage noise-enhancement 316 accelerates activation of the pluripotency program. To confirm the results in an orthogonal 317 reprogramming assay, Oct4-GFP primary MEFs were transduced with retroviral vectors 318 expressing Oct4, Sox2, Klf4, and c-Myc. IdU supplementation for the 48 hours immediately 319 following transduction caused a $\sim 2.4$-fold increase in the number of Oct4-GFP $(+)$ colonies (Fig. $3204 \mathrm{G})$, further demonstrating how amplification of intrinsic gene expression fluctuations can 321 potentiate cell-fate conversion.

324 Overall, these data reveal that a DNA-surveillance pathway exploits the biomechanical link 325 between supercoiling and transcription to homeostatically enhance noise without altering mean326 expression levels. This homeostatic noise-without-mean amplification appears to increase cellular 327 plasticity, thus facilitating reprogramming of cellular identity. This raises intriguing implications 328 for the role of naturally occurring oxidized nucleobases (e.g., hmU) in cell-fate determination, 329 particularly since these base modifications are found at higher frequencies in embryonic stem-cell 330 DNA (57). Mechanistic insight from modeling and experimental perturbation of Apex1 suggest 331 that homeostatic (i.e., orthogonal) noise amplification may also apply to other DNA-processing 332 activities that interrupt transcription. It is important to note that homeostatic noise amplification 333 cannot occur for all promoters (i.e., promoters with $K_{O F F}>>K_{O N}$ are precluded as they will exhibit 334 increased mean) and propagation of transcriptional variability to the protein level likely depends 335 on protein half-lives and thus may not occur for a large swath of proteins. The proteins monitored 336 in this study either have naturally short half-lives (Nanog) or PEST tags (e.g. d 2 GFP) which 337 minimizes the buffering of transcriptional bursts conferred by longer protein half-lives (87). The 338 ability to independently control the mean and variance of gene expression may indicate that cells 339 have the ability to amplify transcriptional noise for fate exploration and specification. 


\section{References and Notes}

1. L. Boltzmann, Weitere Studien uber das Wärmegleichgewicht unter Gasmolekulen, Sitzungsber. Kais. Akad. Wiss. Wien Math. Naturwiss 66, 275-370 (1872).

2. S. Arrhenius, Über die Reaktionsgeschwindigkeit bei der Inversion von Rohrzucker durch Säuren. Zeitschrift für Physikalische Chemie 4, 226 (1889).

3. L. Roberts, Picture coding using pseudo-random noise. IEEE Transactions on Information Theory 8, 145-154 (1962).

4. P. Fatt, B. Katz, Some Observations on Biological Noise. Nature 166, 597-598 (1950).

5. A. A. Priplata, J. B. Niemi, J. D. Harry, L. A. Lipsitz, J. J. Collins, Vibrating insoles and balance control in elderly people. The Lancet 362, 1123-1124 (2003).

6. A. A. Priplata et al., Noise-enhanced balance control in patients with diabetes and patients with stroke. Annals of Neurology 59, 4-12 (2006).

7. A. Aboutorabi, M. Arazpour, M. Bahramizadeh, F. Farahmand, R. Fadayevatan, Effect of vibration on postural control and gait of elderly subjects: a systematic review. Aging Clinical and Experimental Research 30, 713-726 (2018).

8. D. Cohen, Optimizing reproduction in a randomly varying environment. Journal of Theoretical Biology 12, 119-129 (1966).

9. A. Arkin, J. Ross, H. H. McAdams, Stochastic kinetic analysis of developmental pathway bifurcation in phage lambda-infected Escherichia coli cells. Genetics 149, 1633-1648 (1998).

10. J. L. Spudich, D. E. Koshland, Non-genetic individuality: chance in the single cell. Nature 262, 467-471 (1976).

11. N. Q. Balaban, J. Merrin, R. Chait, L. Kowalik, S. Leibler, Bacterial Persistence as a Phenotypic Switch. Science 305, 1622 (2004).

12. P. B. Gupta et al., Stochastic state transitions give rise to phenotypic equilibrium in populations of cancer cells. Cell 146, 633-644 (2011).

13. L. S. Weinberger, J. C. Burnett, J. E. Toettcher, A. P. Arkin, V. D. Schaffer, Stochastic gene expression in a lentiviral positive-feedback loop: HIV-1 Tat fluctuations drive phenotypic diversity. Cell 122, 169-182 (2005).

14. W. J. Blake, M. Kærn, C. R. Cantor, J. J. Collins, Noise in eukaryotic gene expression. Nature 422, 633-637 (2003).

15. I. Golding, J. Paulsson, S. M. Zawilski, E. C. Cox, Real-time kinetics of gene activity in individual bacteria. Cell 123, 1025-1036 (2005). 
16. T. B. Kepler, T. C. Elston, Stochasticity in transcriptional regulation: Origins, consequences, and mathematical representations. Biophysical Journal 81, 31163136 (2001).

17. A. Raj, C. S. Peskin, D. Tranchina, D. Y. Vargas, S. Tyagi, Stochastic mRNA Synthesis in Mammalian Cells. PLoS Biology 4, e309 (2006).

18. J. Peccoud, B. Ycart, Markovian modeling of gene-product synthesis. Theoretical Population Biology 48, 222-234 (1995).

19. J. R. Chubb, T. Trcek, S. M. Shenoy, R. H. Singer, Transcriptional Pulsing of a Developmental Gene. Current Biology 16, 1018-1025 (2006).

20. B. Munsky, G. Neuert, A. Van Oudenaarden, Using Gene Expression Noise to Understand Gene Regulation. Science 336, 183-187 (2012).

21. J. Rodriguez, D. R. Larson, Transcription in Living Cells: Molecular Mechanisms of Bursting. Annual Review of Biochemistry 89, (2020).

22. D. Nicolas, B. Zoller, D. M. Suter, F. Naef, Modulation of transcriptional burst frequency by histone acetylation. Proceedings of the National Academy of Sciences of the United States of America 115, 7153-7158 (2018).

23. A. Bar-Even et al., Noise in protein expression scales with natural protein abundance. Nature Genetics 38, 636-643 (2006).

24. R. D. Dar et al., Transcriptional Bursting Explains the Noise-Versus-Mean Relationship in mRNA and Protein Levels. PLOS ONE 11, e0158298 (2016).

25. J. R. S. Newman et al., Single-cell proteomic analysis of S. cerevisiae reveals the architecture of biological noise. Nature 441, 840-846 (2006).

26. U. Alon, An introduction to systems biology : design principles of biological circuits. (Chapman \& Hall/CRC, Boca Raton, FL, 2007), pp. xvi, 301 p., [304] p. of plates.

27. R. D. Dar, N. N. Hosmane, M. R. Arkin, R. F. Siliciano, L. S. Weinberger, Screening for noise in gene expression identifies drug synergies. Science 344, 1392-1396 (2014).

28. M. M. K. Hansen et al., A Post-Transcriptional Feedback Mechanism for Noise Suppression and Fate Stabilization. Cell 173, 1609-1621 e1615 (2018).

29. Y. Li et al., Noise-driven cellular heterogeneity in circadian periodicity. Proceedings of the National Academy of Sciences 117, 10350-10356 (2020).

30. B. D. Macarthur, I. R. Lemischka, Statistical Mechanics of Pluripotency. Cell 154, 484489 (2013). 
406

407

408

409

410

31. R. M. Kumar et al., Deconstructing transcriptional heterogeneity in pluripotent stem cells. Nature 516, 56-61 (2014).

32. C. Furusawa, K. Kaneko, A Dynamical-Systems View of Stem Cell Biology. Science 338, 215-217 (2012).

33. Zakary et al., Dynamic Heterogeneity and DNA Methylation in Embryonic Stem Cells. Molecular Cell 55, 319-331 (2014).

34. E. Abranches et al., Stochastic NANOG fluctuations allow mouse embryonic stem cells to explore pluripotency. Development 141, 2770-2779 (2014).

35. A. Butler, P. Hoffman, P. Smibert, E. Papalexi, R. Satija, Integrating single-cell transcriptomic data across different conditions, technologies, and species. Nature Biotechnology 36, 411-420 (2018).

36. M. M. K. Hansen, V. R. Desai, M. L. Simpson, L. S. Weinberger, Cytoplasmic Amplification of Transcriptional Noise Generates Substantial Cell-to-Cell Variability. Cell Systems 7, 384-397.e386 (2018).

37. A. Sanchez, I. Golding, Genetic determinants and cellular constraints in noisy gene expression. Science 342, 1188-1193 (2013).

38. D. M. Suter et al., Mammalian genes are transcribed with widely different bursting kinetics. Science 332, 472-474 (2011).

39. N. Eling, A. C. Richard, S. Richardson, J. C. Marioni, C. A. Vallejos, Correcting the Mean-Variance Dependency for Differential Variability Testing Using Single-Cell RNA Sequencing Data. Cell Systems 7, 284-294.e212 (2018).

40. C. A. Vallejos, J. C. Marioni, S. Richardson, BASiCS: Bayesian Analysis of Single-Cell Sequencing Data. PLOS Computational Biology 11, e1004333 (2015).

41. M. Kærn, T. C. Elston, W. J. Blake, J. J. Collins, Stochasticity in gene expression: From theories to phenotypes. Nature Reviews Genetics 6, 451-464 (2005).

42. M. B. Elowitz, A. J. Levine, E. D. Siggia, P. S. Swain, Stochastic gene expression in a single cell. Science 297, 1183-1186 (2002).

43. M. S. H. Ko, A stochastic model for gene induction. Journal of Theoretical Biology 153, 181-194 (1991).

44. P. S. Swain, M. B. Elowitz, E. D. Siggia, Intrinsic and extrinsic contributions to stochasticity in gene expression. Proceedings of the National Academy of Sciences of the United States of America 99, 12795-12800 (2002).

45. A. Scialdone et al., Computational assignment of cell-cycle stage from single-cell transcriptome data. Methods 85, 54-61 (2015). 
46. S. Hooshangi, S. Thiberge, R. Weiss, Ultrasensitivity and noise propagation in a synthetic transcriptional cascade. Proceedings of the National Academy of Sciences of the United States of America 102, 3581-3586 (2005).

47. J. H. Pedraza, A. Van Oudenaarden, Noise propagations in gene networks. Science 307, 1965-1969 (2005).

48. R. Bargaje et al., Cell population structure prior to bifurcation predicts efficiency of directed differentiation in human induced pluripotent cells. Proceedings of the National Academy of Sciences of the United States of America 114, 2271-2276 (2017).

49. M. Mojtahedi et al., Cell Fate Decision as High-Dimensional Critical State Transition. PLoS Biology 14, (2016).

50. C. Sokolik et al., Transcription Factor Competition Allows Embryonic Stem Cells to Distinguish Authentic Signals from Noise. Cell Systems 1, 117-129 (2015).

51. T. Kalmar et al., Regulated fluctuations in Nanog expression mediate cell fate decisions in embryonic stem cells. PLoS Biology 7, (2009).

52. D. W. Austin et al., Gene network shaping of inherent noise spectra. Nature 439, 608-611 (2006).

53. N. Rosenfeld, J. W. Young, U. Alon, P. S. Swain, M. B. Elowitz, Gene regulation at the single-cell level. Science 307, 1962-1965 (2005).

54. A. Sigal et al., Variability and memory of protein levels in human cells. Nature 444, 643-646 (2006).

55. S. Ito et al., Role of Tet proteins in $5 \mathrm{mC}$ to $5 \mathrm{hmC}$ conversion, ES-cell self-renewal and inner cell mass specification. Nature 466, 1129-1133 (2010).

56. W. A. Pastor et al., Genome-wide mapping of 5-hydroxymethylcytosine in embryonic stem cells. Nature 473, 394-397 (2011).

57. T. Pfaffeneder et al., Tet oxidizes thymine to 5-hydroxymethyluracil in mouse embryonic stem cell DNA. Nature Chemical Biology 10, 574-581 (2014).

58. M. Tahiliani et al., Conversion of 5-methylcytosine to 5-hydroxymethylcytosine in mammalian DNA by MLL partner TET1. Science 324, 930-935 (2009).

59. Y. F. He et al., Tet-mediated formation of 5-carboxylcytosine and its excision by TDG in mammalian DNA. Science 333, 1303-1307 (2011).

60. L. Shen et al., Genome-wide analysis reveals TET- and TDG-dependent 5methylcytosine oxidation dynamics. Cell 153, 692-706 (2013). 
472

473

474

475

476

477

478

479

480

481

482

483

484

485

486

487

488

489

490

491

492

493

494

495

496

497

498

499

500

501

502

503

504

505

506

61. E. S. J. Arnér, S. Eriksson, Mammalian deoxyribonucleoside kinases. Pharmacology and Therapeutics 67, 155-186 (1995).

62. B. Demple, T. Herman, D. S. Chen, Cloning and expression of APE, the cDNA encoding the major human apurinic endonuclease: Definition of a family of DNA repair enzymes. Proceedings of the National Academy of Sciences of the United States of America 88, 11450-11454 (1991).

63. T. Lindahl, R. D. Wood, Quality control by DNA repair. Science 286, 1897-1905 (1999).

64. S. Xanthoudakis, R. J. Smeyne, J. D. Wallace, T. Curran, The redox/DNA repair protein, Ref-1, is essential for early embryonic development in mice. Proceedings of the National Academy of Sciences of the United States of America 93, 8919-8923 (1996).

65. S. Madhusudan et al., Isolation of a small molecule inhibitor of DNA base excision repair. Nucleic Acids Research 33, 4711-4724 (2005).

66. O. A. Kladova et al., The role of the N-terminal domain of human apurinic/apyrimidinic endonuclease 1, APE1, in DNA glycosylase stimulation. DNA Repair 64, 10-25 (2018).

67. C. D. Mol, T. Izumi, S. Mitra, J. A. Talner, DNA-bound structures and mutants reveal abasic DNA binding by APE1 DNA repair and coordination. Nature 403, 451-456 (2000).

68. D. R. McNeill, D. M. Wilson, A dominant-negative form of the major human abasic endonuclease enhances cellular sensitivity to laboratory and clinical DNA-damaging agents. Molecular Cancer Research 5, 61-70 (2007).

69. S. Chong, C. Chen, H. Ge, X. S. Xie, Mechanism of transcriptional bursting in bacteria. Cell 158, 314-326 (2014).

70. N. Mitarai, I. B. Dodd, M. T. Crooks, K. Sneppen, The Generation of PromoterMediated Transcriptional Noise in Bacteria. PLoS Computational Biology 4, e1000109 (2008).

71. S. A. Sevier, D. A. Kessler, H. Levine, Mechanical bounds to transcriptional noise. Proceedings of the National Academy of Sciences of the United States of America 113, 13983-13988 (2016).

72. S. Corless, N. Gilbert, Investigating DNA supercoiling in eukaryotic genomes. Briefings in Functional Genomics 16, 379-389 (2017).

73. F. Kouzine, L. Baranello, D. Levens, in Methods in Molecular Biology. (2018), vol. 1703, pp. 95-108. 
74. K. N. Babos et al., Mitigating Antagonism between Transcription and Proliferation Allows Near-Deterministic Cellular Reprogramming. Cell Stem Cell 25, 486-500.e489 (2019).

75. P. Guptasarma, Cooperative relaxation of supercoils and periodic transcriptional initiation within polymerase batteries. BioEssays 18, 325-332 (1996).

76. S. Kim, B. Beltran, I. Irnov, C. Jacobs-Wagner, Long-Distance Cooperative and Antagonistic RNA Polymerase Dynamics via DNA Supercoiling. Cell 179, 106119.e116 (2019).

77. F. Kouzine, S. Sanford, Z. Elisha-Feil, D. Levens, The functional response of upstream DNA to dynamic supercoiling in vivo. Nature Structural and Molecular Biology 15, 146-154 (2008).

78. J. Liu et al., The FUSE/FBP/FIR/TFIIH system is a molecular machine programming a pulse of c-myc expression. The EMBO Journal 25, 2119-2130 (2006).

79. M. Mizutani, T. Ohta, H. Watanabe, H. Handa, S. Hirose, Negative supercoiling of DNA facilitates an interaction between transcription factor IID and the fibroin gene promoter. Proceedings of the National Academy of Sciences of the United States of America 88, 718-722 (1991).

80. S. S. Teves, S. Henikoff, Transcription-generated torsional stress destabilizes nucleosomes. Nature Structural and Molecular Biology 21, 88-94 (2014).

81. M. Bazlekowa-Karaban et al., Mechanism of stimulation of DNA binding of the transcription factors by human apurinic/apyrimidinic endonuclease 1, APE1. DNA Repair 82, (2019).

82. J. F. Breit, K. Ault-Ziel, A.-B. Al-Mehdi, M. N. Gillespie, Nuclear protein-induced bending and flexing of the hypoxic response element of the rat vascular endothelial growth factor promoter. The FASEB Journal 22, 19-29 (2008).

83. M. Delmans, M. Hemberg, Discrete distributional differential expression (D3E) - a tool for gene expression analysis of single-cell RNA-seq data. BMC Bioinformatics 17, 110 (2016).

84. J. K. Kim, J. C. Marioni, Inferring the kinetics of stochastic gene expression from single-cell RNA-sequencing data. Genome Biology 14, 1-12 (2013).

85. W. J. Blake et al., Phenotypic Consequences of Promoter-Mediated Transcriptional Noise. Molecular Cell 24, 853-865 (2006).

86. J. Guo, J. Zheng, HopLand: Single-cell pseudotime recovery using continuous Hopfield network-based modeling of Waddington's epigenetic landscape. Bioinformatics 33, i102-i109 (2017). 
542 87. A. Singh, Transient changes in intercellular protein variability identify sources of

543 noise in gene expression. Biophysical Journal 107, 2214-2220 (2014).

544 88. F. Mueller et al., FISH-quant: Automatic counting of transcripts in 3D FISH images.

$545 \quad$ Nature Methods 10, 277-278 (2013).

546 89. E. P. Nora et al., Targeted Degradation of CTCF Decouples Local Insulation of 547 Chromosome Domains from Genomic Compartmentalization. Cell, (2017).

548 90. P. Angerer et al., Destiny: Diffusion maps for large-scale single-cell data in R.

549 Bioinformatics 32, 1241-1243 (2016).

550 91. M. A. Horlbeck et al., Compact and highly active next-generation libraries for

92. L. V. Sharova et al., Database for mRNA Half-Life of 19977 Genes Obtained by DNA Microarray Analysis of Pluripotent and Differentiating Mouse Embryonic Stem Cells. DNA Research 16, 45-58 (2009).

93. J. Zhang, T. Zhou, Promoter-mediated Transcriptional Dynamics. Biophysical Journal $556 \quad 106,479-488(2014)$.

94. K. P. Burnham, D. R. Anderson, K. P. Burnham, Model selection and multimodel inference : a practical information-theoretic approach. (Springer, New York, ed. 2nd, 2002), pp. xxvi, 488 p.

560 95. C. McQuin et al., CellProfiler 3.0: Next-generation image processing for biology. PLOS $561 \quad$ Biology 16, e2005970 (2018).

96. H. Ochiai, T. Sugawara, T. Sakuma, T. Yamamoto, Stochastic promoter activation affects Nanog expression variability in mouse embryonic stem cells. Scientific Reports 4, 7125 (2015). 


\section{Acknowledgements:}

567 We thank Michael Simpson, Benoit Bruneau, Jonathon Weissman and the Weinberger lab for

568 thoughtful discussions and suggestions. We thank Kathryn Claiborn for editing and Giovanni

569 Maki for graphics support. We would like to acknowledge the technical assistance of Nandhini

570 Raman in the Gladstone Institute Flow Cytometry Facility (NIH S10 RR028962, P30 AI027763,

571 DARPA, and the James B. Pendleton Charitable Trust) and the Gladstone Assay Development

572 and Drug Discovery Core. We also acknowledge Kurt Thorn and DeLaine Larson in the UCSF

573 Nikon Imaging Center (NIH S10 1S10OD017993-01A1). We are grateful to the Gladstone

574 Institute Genomics Core for assistance with single-cell RNA-sequencing experiments. The dual-

575 tagged Sox 2 mESCs were a kind donation from Benoit Bruneau and Elphege Nora. We thank

576 Noam Vardi for the EF- $1 \alpha \mathrm{d}_{2}$ GFP and UBC $\mathrm{d}_{2}$ GFP isoclones. We thank Marco Jost and

577 Jonathon Weissman for CRISPRi reagents. Funding: R.V.D. is supported by an NIH/NICHD

578 F30 fellowship (HD095614-03). L.S.W. acknowledges support from the Bowes Distinguished

579 Professorship, Alfred P. Sloan Research Fellowship, Pew Scholars in the Biomedical Sciences

580 Program, NIH awards R01AI109593, P01AI090935, and the NIH Director's New Innovator

581 Award (OD006677) and Pioneer Award (OD17181) programs. Author contributions: R.V.D.

582 and L.S.W. conceived and designed the study. R.V.D., C.U., S.D., and L.S.W conceived and

583 designed the cellular reprogramming experiments. R.V.D. and C.U. performed the experiments.

584 R.V.D., M.M.K.H., and B.M. analyzed data. R.V.D., M.M.K.H., B.M. and L.S.W. constructed

585 and analyzed the mathematical models. R.V.D. and L.S.W. wrote the manuscript. Competing

586 interests: Authors declare no competing interests. Data and materials availability:

587 Sequencing data from bulk RNA-seq and single-cell RNA-seq will be deposited onto GEO.

588 Custom code for analysis of sequencing data and mathematical modeling will be made available

589 on GitHub. Reagents, including plasmids and cell-lines, are available from the corresponding

590 author upon request.

591 Supplementary Materials:

592 Materials and Methods

593 Supplementary Text

594 Figures S1-S27

595 Tables S1-S6

596 References (88-96) 
597 Figure 1: Genome-wide amplification of cell-to-cell mRNA variability (i.e., 'noise') 598 independent of mean.

599 A. (Left) Monte-Carlo simulations of the two-state Random-Telegraph model of transcription 600 showing low noise and higher noise trajectories with matched mean expression levels. Coefficient 601 of Variation $\left(\sigma^{2} / \mu^{2}, \mathrm{CV}^{2}\right)$ quantifies magnitude of fluctuations. (Right) The predicted facilitation 602 of state transitions through 'dithering'. B. (Top) Schematic of two-state Random-Telegraph 603 model of transcription. (Bottom) Schematic of mean vs. CV ${ }^{2}$ for mRNA abundance with solid 604 gray line representing Poisson, inverse scaling of $\mathrm{CV}^{2}$ as a function of mean. Question mark 605 symbolizes unknown noise-control mechanisms that amplify fluctuations independently of mean. 606 Histograms depict expected shift in mRNA copy number distributions. C-F. scRNA-seq of 607 mESCs treated with DMSO (black) or $10 \mu \mathrm{M}$ IdU (red) for 24h. 812 and 744 transcriptomes 608 (filtered and normalized with Seurat) from DMSO and IdU treatments, respectively, were 609 analyzed. (C) Mean expression vs. $\mathrm{CV}^{2}$ and (D) mean vs. variance for 4,578 genes. Four examples 610 of housekeeping genes (purple) demonstrate how IdU increases expression fluctuations with 611 minimal change in mean (white arrows). (E) Mean expression and (F) Fano factor $\left(\sigma^{2} / \mu\right)$ of 4,578 612 genes in DMSO vs. IdU treatments. Overlay of density contours reveals how center of mass lies 613 on diagonal for mean values while lying above the diagonal for Fano factor measurements. G-H. 614 BASiCS analysis of scRNA-seq data for 4,578 genes. (G) Fold change in mean vs. certainty 615 (posterior probability) that gene is up- or down-regulated. With IdU treatment, 113 genes (red) 616 were classified as differentially expressed ( $>2$-fold change in mean with $>85 \%$ probability). (H)

617 Fold change in over-dispersion vs. certainty (posterior probability) that gene is highly- or lowly618 variable. 945 genes (red) were classified as highly variable ( $>1.5$-fold change in over-dispersion 619 with $>85 \%$ probability). 
620 Figure 2: Amplification of mRNA noise is not due to extrinsic sources, results from shorter 621 but more intense transcriptional bursts, and propagates to protein levels.

622 A. Pearson correlations of expression for gene pairs in scRNA-seq dataset. Hierarchical clustering 623 reveals networks of genes (highlighted in black rectangles) sharing similar correlation patterns.

624 Dashed rectangle highlights network enriched with pluripotency factors like Nanog. IdU treatment causes a fading of heatmap, indicating weakened expression correlations. B-D. Results of 626 smRNA-FISH used to count nascent and mature Nanog mRNA in Nanog-GFP mESCs treated with DMSO or $10 \mu \mathrm{M}$ IdU for 24 hours in $2 \mathrm{i} / \mathrm{LIF}$ media. Data are from four biological replicates. (B) (Left) Representative micrograph (maximum intensity projection) in which Nanog transcripts are labelled with probe-set for eGFP. Bright foci correspond to transcriptional centers as verified by intron probe set. Scale bar is $5 \mu \mathrm{m}$. (Right) Distributions of mature Nanog transcripts/cell. Dashed lines represent mean. IdU treatment increases cell-to-cell variability of transcript 632 abundance as reported by averaged Fano factors $( \pm \mathrm{SD}),{ }^{*} \mathrm{p}=0.0011$ by a two-tailed, unpaired

633 Student's t test. (C) Fraction of possible transcriptional centers that are active as detected by 634 overlap of signal in exon and intron probe channels. Each cell is assumed to have 2 possible 635 transcriptional centers (TCs). Data represent mean and SD. With IdU, the fraction of possible of 636 TCs that are active decreases, ${ }^{* *} \mathrm{p}=6.9 \times 10^{-5}$ by a two-tailed, unpaired Student's t test. (D) 637 Distributions of nascent Nanog mRNA per TC. With IdU, active TCs have more nascent mRNAs, $638{ }^{* *} \mathrm{p}=1.0 \times 10^{-4}$ by a two-tailed, unpaired Student's t test. E. Representative flow cytometry 639 distribution of Nanog-GFP expression in mESCs treated with DMSO or $10 \mu \mathrm{M}$ IdU for $24 \mathrm{~h}$ in 640 2i/LIF. Dashed lines represent mean. Fold change in Fano factor $( \pm \mathrm{SD})$ obtained from three 641 biological replicates. IdU increases cell-to-cell variability in Nanog protein expression. Inset: 642 Representative flow cytometry dot-plot showing conservative gating on forward and side scatter 643 to filter extrinsic noise arising from cell size heterogeneity. F. Time-lapse imaging of Nanog644 GFP mESCs treated with either DMSO $(n=1513)$ or $10 \mu \mathrm{M}$ IdU $(n=1414)$ in $2 \mathrm{i} / \mathrm{LIF}$. Image 645 acquisition began immediately after addition of compounds. Trajectories from two replicates of 646 each condition are pooled, with solid and dashed lines representing mean and standard deviation 647 of trajectories respectively. Distributions of Nanog-GFP represent expression at final time-point. 648 Intrinsic- $\mathrm{CV}^{2}$ of each detrended trajectory was calculated, with the average ( \pm SD) of all 649 trajectories reported. 
650 Figure 3: Noise amplification independent of mean is due to Apex1-mediated DNA repair

651 A. Screening of 14 additional nucleoside analogs. Nanog-GFP mESCs grown in 2i/LIF were 652 supplemented with $10 \mu \mathrm{M}$ of nucleoside analog for $24 \mathrm{~h}$. Fano factor for Nanog protein expression 653 was normalized to DMSO. Data represent mean ( \pm SD) of two biological replicates. BrdU, hmC 654 and hmU increase Nanog expression variability as compared to DMSO, ${ }^{*} \mathrm{p}<0.01$ by a KruskalWallis test followed by Tukey's multiple comparison test. B. Schematic of nucleoside analog 656 incorporation into genomic DNA and removal via base excision repair pathway. C. (Left) CRISPRi screening for genetic dependencies of IdU noise enhancement. Nanog-GFP mESCs stably expressing dCas9-KRAB-p2A-mCherry were transduced with a single gRNA expression vector with BFP reporter. 75 gRNAs ( 25 genes, 3 gRNAs/gene) were tested in addition to 3 nontargeting control gRNAs. Two days following transduction, each gRNA-expressing population of mESCs was treated with DMSO or $10 \mu \mathrm{M}$ IdU for $24 \mathrm{~h}$ in $2 \mathrm{i} / \mathrm{LIF}$ media. Nanog-GFP protein 662 expression was measured for mCherry/BFP double positive cells. Nanog Fano factor for DMSO 663 and IdU treatment of each gRNA population was normalized to Nanog Fano factor of nontargeting gRNA+DMSO population. Each point represents a gRNA. Dashed horizontal line 665 represents average noise enhancement of Nanog from IdU in the background of non-targeting 666 gRNA expression (black squares). Knockdown of Apex1 and Tk1 diminishes noise enhancement of Nanog from IdU. (Right) Representative flow cytometry distributions of Nanog expression for mESCs expressing non-targeting (top-right), Apex1 (middle-right), or Tk1 (bottom-right) gRNAs and treated with DMSO or $10 \mu \mathrm{M}$ IdU. D. Combination of IdU and small-molecule inhibitor of the Apex 1 endonuclease domain (CRT0044876). (Left) mESCs were treated with DMSO, 100 $\mu \mathrm{M}$

671 CRT0044876, $10 \mu \mathrm{M}$ IdU or $10 \mu \mathrm{M}$ IdU $+100 \mu \mathrm{M}$ CRT0044876 for $24 \mathrm{~h}$ in 2i/LIF. Nanog Fano

672 factor for each treatment was normalized to DMSO control. Data represent mean ( \pm SD) of three 673 biological replicates. Inhibition of Apex1 endonuclease domain in combination with IdU 674 synergistically increases cell-to-cell variability of Nanog expression, ${ }^{*} \mathrm{p}=0.0028$ by a two-tailed, 675 unpaired Student's t test. (Right) Representative flow cytometry distributions of Nanog expression 676 for mESCs treated with DMSO or $10 \mu \mathrm{M}$ IdU $+100 \mu \mathrm{M}$ CRT0044876. E. Single-cell 677 quantification of negative supercoiling levels using psoralen-crosslinking assay. mESCs were 678 treated with DMSO, $10 \mu \mathrm{M}$ IdU or $10 \mu \mathrm{M}+100 \mu \mathrm{M}$ CRT0044876 for $24 \mathrm{~h}$ in $2 \mathrm{i} / \mathrm{LIF}$. $1 \mu \mathrm{M}$ 679 aphidicolin was added to cultures $2 \mathrm{~h}$ prior to incubation with biotinylated-trimethylpsoralen 680 (bTMP). Following UV-crosslinking, cells were stained with streptavidin-Alexa594 conjugate 
681 and DAPI. Distributions for nuclear intensities of bTMP staining are shown. Data are pooled 682 from two biological replicates of each treatment. IdU treatment increases negative supercoiling as 683 compared to DMSO control, ${ }^{* *} \mathrm{p}<0.0001$. IdU in combination with CRT0044876 further increases 684 supercoiling levels as compared to DMSO $\left({ }^{* *} \mathrm{p}<0.0001\right)$ and $\operatorname{IdU}$ alone $\left({ }^{* *} \mathrm{p}<0.0001\right)$. P values 685 were calculated using Kruskal-Wallis test followed by Tukey's multiple comparison test. F. 686 CRISPRi Knockdown of Topoisomerases involved in relaxation of DNA supercoiling. Nanog 687 Fano factor was normalized to scrambled gRNA population. Data represent mean $( \pm \mathrm{SD})$ of three 688 biological replicates. Knockdown of Top1 $(* p=0.002)$ and Top2a $(* p=0.003)$ increases Nanog 689 expression variability. P values were calculated by two-tailed, unpaired Student's t test. 
Ela Ure 6 oRxiv preprint doi: https://doi.org/10.1101/2020.06.29.128439; this version posted June 30, 2020. The copyright holder for this preprint (which was not certified by peer review) is the author/funder, who has granted bioRxiv a license to display the preprint in perpetuity. It is made
available under aCC-BY-NC-ND 4.0 International license.

Model 5

Transcription-Coupled Repair

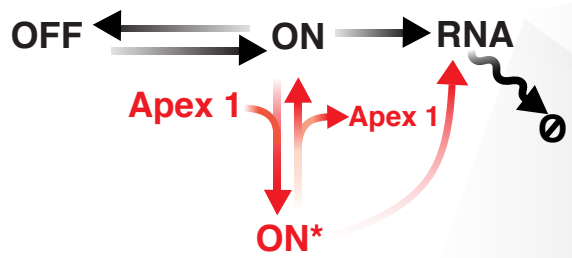

B

Macroscopic Behavior

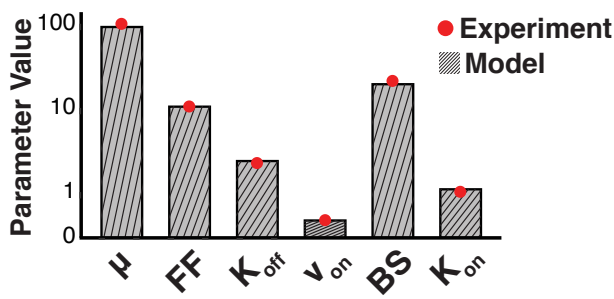

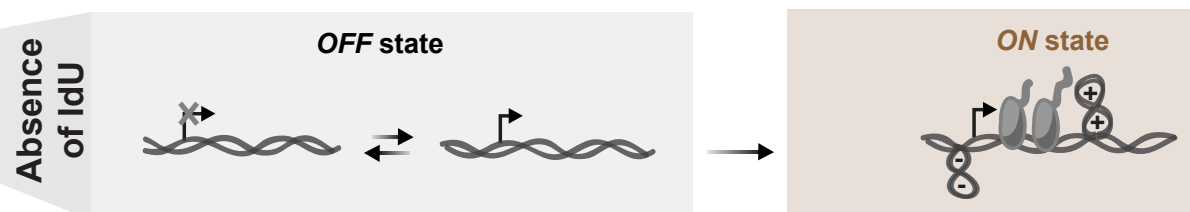

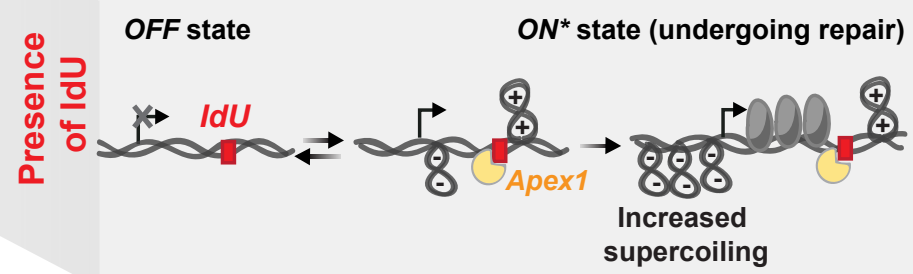

ON state (Repaired)

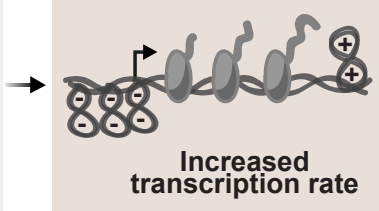

\section{Experimental Validation of Model}
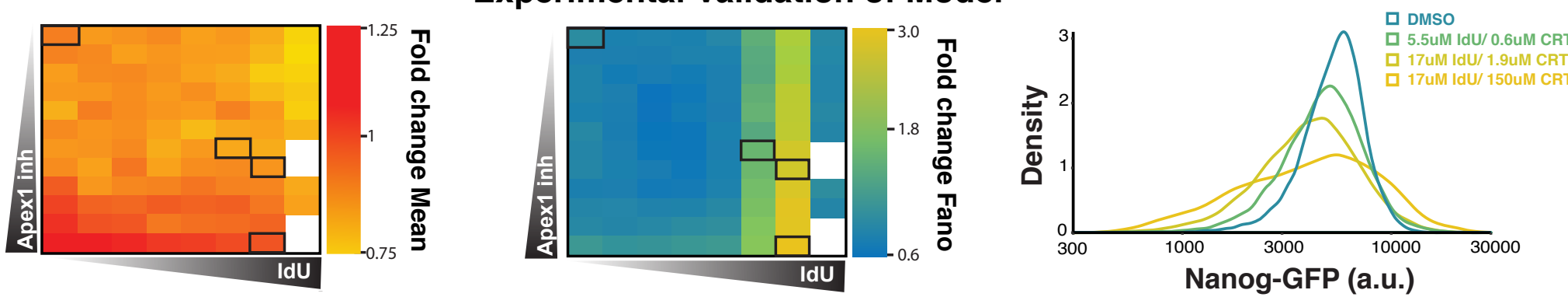

D
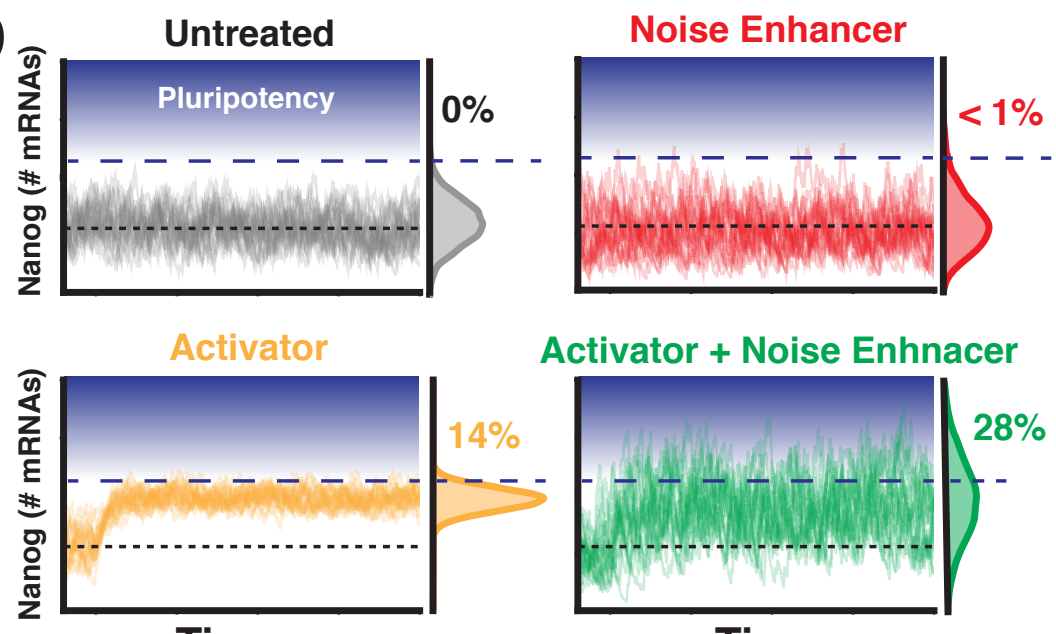

Activator + Noise Enhnacer

Time

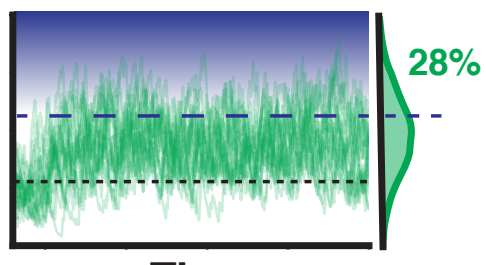

E

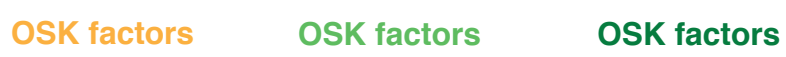

$+$

DMSO $\quad 1 \mathrm{uM} \mathrm{IdU}(48 \mathrm{~h})$

4uM IdU (48h)

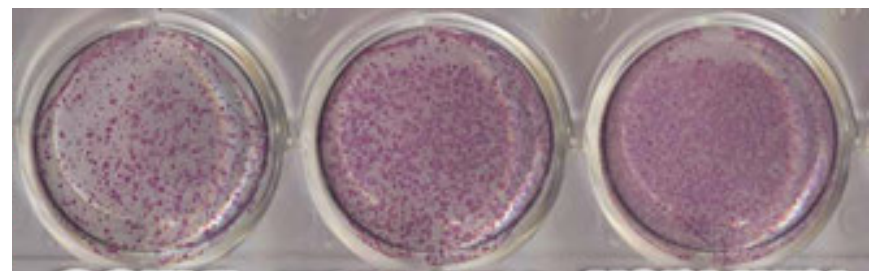

\section{Time}

E

8

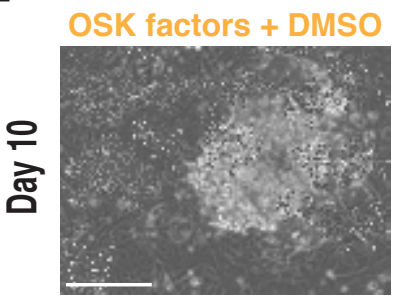

$\frac{7}{\frac{0}{0}}$

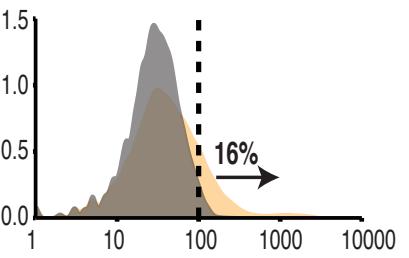

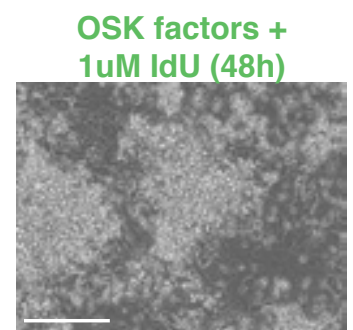

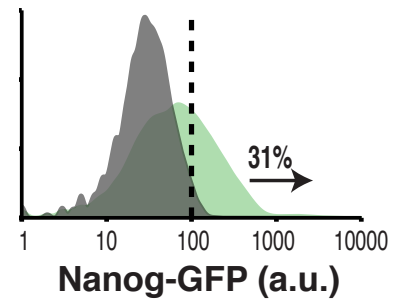

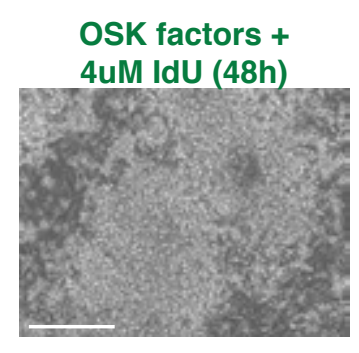

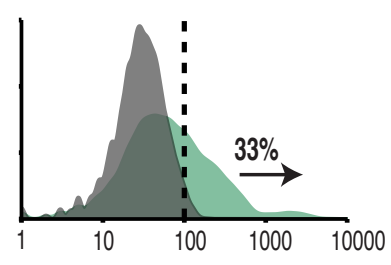

$G$

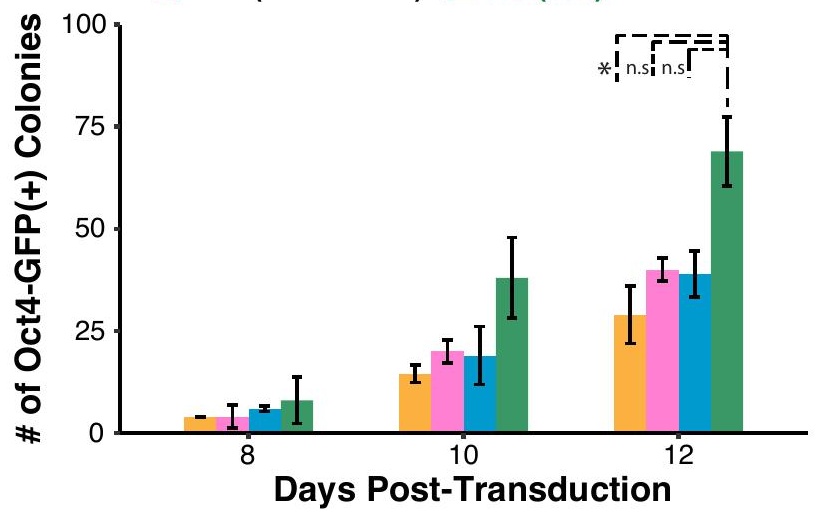


Figure 4: Transcription-coupled DNA repair tunes transcriptional bursting across a broad parameter regime, generating amplified noise which potentiates reprogramming of cellular identity

693 A. Detailed schematic of Model 5 (see Fig. S20 for schematic of models 1-4), which represents 694 transcription-coupled repair (TCR). In the presence of IdU (bottom panel), Apex1 binding occurs when gene is transcriptionally permissive ( $\mathrm{ON}$ state). Binding induces negative supercoiling which lengthens the time that a gene is transcriptionally non-productive ( $\mathrm{ON}^{*}$ state) while also facilitating recruitment of transcriptional resources. Upon repair completion, a higher transcriptional rate compensates for lost productivity. Mean expression is maintained with larger transcriptional fluctuations. B. The macroscopic behavior (mean Nanog mRNA $[\mu]$, Fano factor

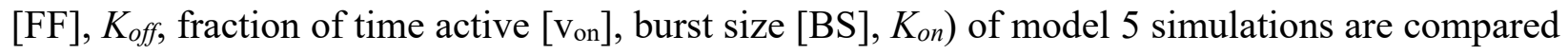
to experimentally derived values of each parameter (red dots) obtained from smRNA-FISH data. Absolute percentage error (APE) is calculated as described in supplementary text 5.2.2. Model 5 (TCR model) best matches experimental data. C. Testing of 96 concentration combinations of IdU and CRT0044876 to validate tunability of Nanog variability. IdU and CRT0044876 were used to increase binding and decrease unbinding of Apex1 respectively. Nanog-GFP mESCs grown in 96-well plates were treated with 12 concentrations of CRT0044876 ranging from 0 to $150 \mu \mathrm{M}$ in combination with 8 concentrations of IdU ranging from 0 to $50 \mu \mathrm{M}$. Data represent average of two biological replicates. (Leftmost and Center Panels) 96-well heatmaps displaying fold change in Nanog mean and Fano factor for each drug combination as compared to DMSO (top-leftmost well). Insufficient number of cells $(<50,000)$ for extrinsic noise filtering were recorded from white wells. (Rightmost Panel) Representative flow cytometry distributions from highlighted wells (black rectangles). Nanog variability increases independently of the mean. D.

713 Simulations of the TCR model for Nanog gene expression in the presence of DMSO (top left), IdU

714 (top right), an activator (increased $K_{O N}$, decreased $K_{O F F}$ ) of promoter activity (bottom left) and an

715 activator combined with IdU (bottom right). Homeostatic noise amplification potentiates

716 responsiveness to an activator of gene expression as demonstrated by increased threshold crossing

717 (14\% to $28 \%$ ). E. Nanog-GFP secondary MEFs (seeded at 10,000 cells $/ \mathrm{cm}^{2}$ ) harboring stably718 integrated, doxycycline-inducible cassettes for Oct4, Sox2, and Klf4 (OSK) were subjected to 10 719 days of doxycycline treatment in combination with DMSO (first well), $1 \mu \mathrm{M}$ IdU (second well), or $4 \mu \mathrm{M}$ IdU (third well) for the first 48 hours of reprogramming. Alkaline phosphatase staining for 
721 pluripotent colonies of cells demonstrates how IdU treatment potentiates pluripotency induction.

722 F. (Top) Micrographs of Nanog-GFP secondary MEFs at day 10 of doxycycline-induced

723 reprogramming $($ scale $b a r=100 \mu \mathrm{m})$. (Bottom) Flow cytometric analysis of Nanog-GFP activation

724 at day 10 of reprogramming. Data are pooled from two replicates. G. Oct4-GFP primary MEFs

725 (seeded at 10,000 cells $/ \mathrm{cm}^{2}$ ) were retrovirally transduced with cDNAs encoding Oct4, Sox2, K1f4,

726 and c-Myc. 24 hours after transduction, infected cells were treated with DMSO (continuously),

$7271 \mathrm{mM}$ valproic acid (VPA, continuously), $10 \mu \mathrm{M}$ forskolin (continuously), or $4 \mu \mathrm{M}$ IdU (first 48

728 hours). VPA and forskolin are established enhancers of cellular reprogramming. The number of

729 Oct4-GFP(+) stem cell colonies were counted 8, 10, and 12 days from the start of drug treatment.

730 Data represent mean and SD of 2 biological replicates. Treatment of transduced MEFs with IdU

731 during early stages of reprogramming increases the number of Oct4-GFP(+) colonies that form as

732 compared to DMSO control, $* \mathrm{p}=0.039$ by one-way ANOVA with Bonferroni post hoc test. 


\section{Supplementary Materials for}

\section{Discovery of a Cellular Mechanism Regulating Transcriptional Noise}

Ravi V. Desai, Maike M.K. Hansen, Benjamin Martin, Chen Yu, Sheng Ding, Matt Thomson, Leor S. Weinberger

Correspondence to leor.weinberger@gladstone.ucsf.edu

This PDF file includes:

Materials and Methods

Supplementary Text

Figs. S1 to S27

Captions for Tables S1 to S6 


\section{Materials and Methods}

\section{$\underline{\text { Cell Culture and Growth Conditions }}$}

Mouse E14 embryonic stem cells (male) were routinely cultured in feeder-free conditions on gelatin-coated plates with ESGRO-2i medium (Millipore, cat:SF016-200) at $37^{\circ} \mathrm{C}, 5 \% \mathrm{CO}_{2}$, in humidified conditions (36). Jurkat T Lymphocytes (male) were cultured in RPMI-1640 medium (supplemented with L-glutamine, $10 \%$ fetal bovine serum, and $1 \%$ penicillin-streptomycin), at $37^{\circ} \mathrm{C}$, $5 \% \mathrm{CO}_{2}$, in humidified conditions at $0.1 \times 10^{6}$ to $1 \times 10^{6}$ cells $/ \mathrm{mL}$. Isoclonal Jurkat T Lymphocytes with lentivirally integrated $\mathrm{EF} 1 \alpha-\mathrm{d}_{2}$ GFP construct were previously described (36). Human immortalized myelogenous leukemia (K652, female) cells were cultured in RPMI-1640 medium (supplemented with L-glutamine, $10 \%$ fetal bovine serum, and $1 \%$ penicillin-streptomycin), at $37^{\circ} \mathrm{C}, 5 \% \mathrm{CO}_{2}$, in humidified conditions at $2 \times 10^{5}$ to $2 \times 10^{6}$ cells $/ \mathrm{mL}$. Isoclonal $\mathrm{K} 562$ cells with lentivirally integrated EF $1 \alpha-\mathrm{d}_{2}$ GFP and UBC- $\mathrm{d}_{2}$ GFP constructs were previously described (36). Human embryonic kidney (HEK293, female) cells were cultured in DMEM (supplemented with $10 \%$ fetal bovine serum, $1 \%$ penicillin-streptomycin, 25mM HEPES, and 2mM L-glutamine) at $37^{\circ} \mathrm{C}, 5 \% \mathrm{CO}_{2}$, in humidified conditions at 30 to $90 \%$ confluency.

$\underline{\text { Noise Enhancer Testing on Isoclonal Jurkat and K562 Cells }}$

Jurkat and K562 cells were seeded into 12-well plates at densities of $0.2 \times 10^{6}$ and $0.4 \times 10^{6}$ cells/mL respectively in media containing $20 \mu \mathrm{M}$ IdU (Sigma, cat:I7125, dissolved in DMSO) or equivalent volume of DMSO for 24 hours. Flow cytometry was performed using a BD LSRII cytometer. Treated cells were run unfixed and live to avoid additional sources of variability from fixation. 50k live cells were collected from each sample for noise measurements. Conservative gating for a live subset of approximately $3 \mathrm{k}$ cells of similar size, volume, and state, was applied on the FSC vs. SSC to reduce extrinsic noise contributions as previously described $(25,27)$.

Single-Cell RNA Sequencing Preparation and Analysis

$1 \times 10^{6} \mathrm{mESCs}$ were seeded in a gelatin-coated, $10 \mathrm{~cm}$ dish in $2 \mathrm{i} /$ LIF media. 24 hours following seeding, cultures were replenished with $2 \mathrm{i} / \mathrm{LIF}$ media containing $10 \mu \mathrm{M}$ IdU or an equivalent volume of DMSO for 24 hours. After treatment, cells were trypsinized with TrypLE and spun down for 5 minutes at $200 \mathrm{x}$ g. Single-cell suspensions were prepared in DPBS at a concentration of 83,000 cells/ml. Approximately 3000 cells from each sample were loaded into a chip and processed with the Chromium Single Cell Controller (10x Genomics). To generate single-cell gel beads in emulsion (GEMs), DMSO- and IdU- treated samples were assigned unique indexes using Single Cell 3/ Library and Gel Bead Kit V2 (10x Genomics, cat:120237). Sequencing was performed on an Illumina HiSeq4000 with a paired-end setup specific for 10x libraries.

Data were aligned to mm10 reference genome using 10x Cell Ranger v2. Quality control, normalization and analysis were carried out using two packages: Seurat and BASiCS. For analysis 
in Seurat, gene-barcode matrices were filtered and normalized using the "LogNormalize" method, resulting in 812 and 744 transcriptomes from DMSO and IdU samples. Transcript variability was quantified using variance $\left(\sigma^{2}\right)$, coefficient of variation $\left(\frac{\sigma^{2}}{\mu^{2}}\right)$, and Fano factor $\left(\frac{\sigma^{2}}{\mu}\right)$. During the normalization procedure in Seurat, counts for the $i^{\text {th }}$ gene in the $j^{\text {th }}$ cell $\left(x_{i j}\right)$ are multiplied by the following scaling factor: $S=\frac{10000}{\sum_{i=1}^{n} x_{i j}}$, where $\mathrm{n}$ is the number of genes in the dataset. The scaling factor is therefore dependent on the number of UMIs detected per cell. The coefficient of variation is insensitive to this scaling factor as it is a dimensionless quantity (i.e, $\sigma$ and $\mu$ are scaled by the same factor and thus cancel out when calculating coefficient of variation). However, the Fano factor, which has units, must be re-scaled to account for the differential effect that this normalization procedure has on $\sigma^{2}$ vs. $\mu$ (i.e., $\sigma^{2}$ gets scaled by $S^{2}$ while $\mu$ gets scaled by $S$ ). To negate the carryover of this scaling factor, calculated Fano factors from the Seurat-normalized dataset were multiplied by $\frac{1}{S}$ where $S$ is a unique value for the DMSO and IdU samples: 10000 On average, 4151.3 and 4191.4 UMIs were detected per cell in DMSO and IdU samples respectively.

For analysis using BASiCS, quality control and filtering was performed using the BASiCS_Filter function resulting in an identical number of transcriptomes (812 and 744) as produced by Seurat. Posterior estimates of mean and over-dispersion for each gene were computed using a Markov Chain Monte Carlo (MCMC) simulation with 40,000 iterations and a log-normal prior. For differential mean testing, a threshold of fold change $>2$ with an FDR cutoff of 0.05 was used. Differential variability was tested with a threshold of fold change $>1.5$ with an FDR cutoff of 0.05 . Only genes with no change in mean expression $(4,458$ of 4,578) were considered for interpreting changes in variability.

Gene features and sequences from the GRCm38 reference were used for analysis of gene characteristics that potentiate noise enhancement. TAD boundary locations in mESCs were taken from Hi-C maps produced by Elphège et al (89). DAVID v6.8 was used to test for gene ontology (GO) enrichment among highly variable genes. All tested genes $(4,458)$ from BASiCS were used as background. Bonferroni-corrected p-values (adjusted p-values) were used to visualize GO enrichment. Cell cycle determination was performed using cyclone as implemented in scran (45). The default set of cell cycle marker genes for mESCs (mouse_cycle_markers.rds) was used. Cells were assigned to G1, S, and G2/M phases using their normalized genes counts produced by Seurat. Pseudotime analysis was conducted using destiny (90) with the Seurat-normalized cell-gene matrix as input. Gene-gene correlation matrices were assembled by first filtering out genes from the Seurat-normalized matrix whose mean abundance $<1$ in each treatment group to avoid spurious correlations that may emerge from low expression. 961 genes remained for downstream analysis. Pearson correlation for each gene pair was calculated. Clustering of gene-pairs based on similarity in correlation patterns was performed using the hierarchical clustering method within the seriation package. Change in correlation strength was calculated by subtracting absolute value of gene-pair correlation in DMSO condition from IdU condition.

$\underline{\text { Bulk RNA Sequencing Preparation and Analysis }}$ 
$2 \times 10^{5}$ mESCs were seeded in each well of a gelatin-coated, 6-well plate in 2i/LIF media. 24 hours following seeding, cultures were replenished with $2 \mathrm{i} / \mathrm{LIF}$ media containing $10 \mu \mathrm{M}$ IdU, $5 \mu \mathrm{M}$ IdU or an equivalent volume of DMSO in triplicate for 24 hours. After treatment, cells were trypsinized with TrypLE and RNA was extracted using a RNeasy minikit (Qiagen) according to manufacturer's instructions. ERCC spike-in RNA ( $2 \mu$ l diluted at 1:100) was added to each RNA extraction (Ambion, cat:4456740). A total of 9 cDNA libraries were prepared with an NEBNext Ultra II RNA Library Prep kit (NEB, cat:E7770S) and sequenced with an Illumina HiSeq4000. Sequencing yielded a median of $\sim 40$ million single-end reads per library. Read quality was checked via FASTQC. Reads were aligned to an edited version of the mm10 reference genome containing the ERCC spike-in sequences using TopHat with default parameters. Transcript level quantification was performed using Cufflinks with default parameters. The quantification matrix was then imported into R and analyzed via DESeq2. Samples were normalized using ERCC transcripts as controls for size factor estimation. Differential mean testing was conducted with a threshold of fold change $>2$ and an FDR cutoff of 0.05 .

Single Molecule RNA FISH

Probes for detection of nascent and mature Nanog transcripts were developed using the designer tool from Stellaris (LGC Biosearch Technologies) (Table S1). 30 probes (TAMRA conjugated) for mature Nanog mRNA were targeted towards the 3/ GFP segment of transcripts. 48 probes (Quasar 670 conjugated) for nascent Nanog mRNA were targeted towards the first intronic sequence as taken from the mm10 genome reference. Probes were designed using a masking level of 5 , and at least 2 base pair spacing between single probes.

$1 \times 10^{5}$ Nanog-GFP mESCs were seeded into each well of a gelatin-coated, $35 \mathrm{~mm}$ Ibidi dish (quad-chambered, cat:80416) in 2i/LIF media. 24 hours following seeding, media was replaced with $2 \mathrm{i} / \mathrm{LIF}$ containing $10 \mu \mathrm{M}$ IdU or equivalent volume DMSO. After 24 hours of treatment, cells were then fixed with DPBS in $4 \%$ paraformaldehyde for 10 minutes. Fixed cells were washed with DPBS and stored in $70 \% \mathrm{EtOH}$ at $4^{\circ} \mathrm{C}$ for one hour to permeabilize the cell membranes. Probes were diluted 200 -fold and allowed to hybridize at $37^{\circ} \mathrm{C}$ overnight. Wash steps and DAPI (Thermo) staining were performed as described (https://www.biosearchtech.com/support/resources/stellarisprotocols).

To minimize photo-bleaching, cells were imaged in a buffer containing 50\% glycerol (Thermo), $75 \mu \mathrm{g} / \mathrm{mL}$ glucose oxidase (Sigma Aldrich), $520 \mu \mathrm{g} / \mathrm{mL}$ catalase (Sigma Aldrich), and $0.5 \mathrm{mg} / \mathrm{mL}$ Trolox (Sigma Aldrich). Images were taken on a Zeiss Axio Observer Z1 microscope equipped with a Yokogawa CSU-X1 spinning disk unit and 100x/1.4 oil objective. Approximately 20 xy locations were randomly selected for each condition. For each xy location, Nyquist sampling was performed by taking 30, 0.4 $\mu \mathrm{M}$ steps along the z-plane.

Image analysis and spot counting was performed using FISH-quant (88). Cells were manually 
segmented and analysis was conducted on cells of a similar size to minimize extrinsic noise. Transcriptional centers (TCs) were identified by signal overlap in exon, intron and DAPI channels. The amount of nascent mRNA at TCs was quantified through a weighted superposition of point spread functions.

$\underline{\text { Rate calculations for random-telegraph model }}$

From smRNA-FISH data for Nanog, the kinetic parameters of the random-telegraph model were inferred using the empirically derived values of mRNA mean $(\mu)$, mRNA Fano factor (Fano), transcriptional center frequency $\left(f_{O N}\right)$ and transcriptional center size $\left(T C_{m R N A}\right)(36)$. The transcription rate $\left(k_{t x}\right)$ is calculated as:

$$
k_{t x}=T C_{m R N A} \frac{k_{\text {elongation }}}{L}
$$

where $k_{\text {elongation }}$ is the elongation rate of RNAPII $(1.9 \mathrm{~kb} / \mathrm{min})(96)$ and $L$ is the length of the transcribed region of Nanog. The degradation rate $\left(k_{\text {decay }}\right)$ is calculated as:

$$
k_{\text {decay }}=\frac{f_{O N} \cdot k_{t x}}{\mu}
$$

The rate of promoter activation $\left(k_{O N}\right)$ is given by:

$$
k_{\text {ON }}=k_{\text {decay }}\left(-\frac{\mu\left(f_{\text {ON }}-1\right)+f_{\text {ON }}(\text { Fano }-1)}{\text { Fano }-1}\right)
$$

The rate of promoter inactivation $\left(k_{O F F}\right)$ is given by:

$$
k_{\text {OFF }}=-k_{\text {decay }}\left(-\frac{\mu\left(f_{\text {ON }}-1\right)+f_{\text {ON }}(\text { Fano }-1)}{\text { Fano }-1}\right) \cdot\left(\frac{1}{f_{\text {ON }}}-1\right)
$$

$\underline{\text { Extrinsic Noise Filtering on Flow Cytometry Data }}$

All flow cytometry data were collected on BD FACSCalibur, LSRII or LSRFortessa X-20 with 488-nm laser used to detect GFP. For all measurements of Nanog-GFP mean and variability, $>50 \mathrm{k}$ cells are collected per sample. Gating of cytometry data was performed with FlowJo. Prior to quantification of Nanog-GFP mean and variability, the smallest possible forward- and side-scatter region containing at least $3 \mathrm{k}$ cells was used to isolate cells of similar size and shape. This filters out gene expression variability arising from cell-size heterogeneity as previously established $(25,27,36)$.

$\underline{\text { Cell-cycle Analysis by Propidium-Iodide Staining }}$ 
$2 \times 10^{5}$ Nanog-GFP mESCs were seeded in each well of a gelatin-coated, 6 -well plate in 2i/LIF media. 24 hours following seeding, media was replaced with 2i/LIF media containing $10 \mu \mathrm{M}$ IdU or an equivalent volume of DMSO in triplicate for 24 hours. After treatment, cells were washed with DPBS, dissociated with TrypLE, pelleted, washed with DPBS, and resuspended in ice-cold $70 \%$ ethanol. Samples were stored overnight at $-20^{\circ} \mathrm{C}$ and pelleted the following day at $200 \mathrm{~g}$ for 5 minutes at $4^{\circ} \mathrm{C}$. Cells were washed twice with DPBS supplemented with $0.5 \% \mathrm{BSA}$ to prevent cell loss. Pellets were resuspended in $150 \mu \mathrm{L}$ of DPBS supplemented with $0.1 \mathrm{mg} / \mathrm{ml}$ RNAse A (Thermo) and $30 \mu \mathrm{g} / \mathrm{ml}$ Propidium Iodide (Thermo). After overnight incubation at $4{ }^{\circ} \mathrm{C}$, cells were directly analyzed on a BD LSRII cytometer.

Noise Enhancer Testing in Serum/LIF culture

Serum/LIF media was prepared with 85\% DMEM (supplemented with 2mM of L-glutamine), $15 \%$ FBS, 0.1mM 2-mercaptoethanol, and 1000U/ml of LIF (Sigma Aldrich). Nanog-GFP mESCs grown feeder-free in $2 \mathrm{i} / \mathrm{LIF}$ were passaged and seeded onto gelatin-coated $10 \mathrm{~cm}$ dishes in serum/LIF media. Cells were passaged twice in serum/LIF media prior to noise enhancer testing. $4 \times 10^{5}$ Nanog-GFP mESCs were seeded into each well of a gelatin-coated 6-well plate in serum/LIF media. 24 hours following seeding, media was replaced with serum/LIF supplemented with either $10 \mu \mathrm{M}$ IdU or equivalent volume DMSO in triplicate. After 24 hours of treatment, cells were run unfixed and live on BD LSRII flow cytometer.

Sox 2 two-color reporter assay

The endogenous alleles of Sox2 are tagged with P2A-mClover and P2A-tdTomato. Both fluorophores have a PEST tag, thus shortening their half-lives to approximately 2.5 hours. $2 \times 10^{5}$ Sox2-dual-tag mESCs were seeded in each well of a gelatin-coated, 6-well plate in 2i/LIF media. 24 hours following seeding, cultures were replenished with $2 \mathrm{i} / \mathrm{LIF}$ media containing $10 \mu \mathrm{M}$ IdU or an equivalent volume of DMSO in triplicate for 24 hours. Cells were run unfixed and live on BD LSRII flow cytometer. Intrinsic noise was calculated as in Elowitz et. al (42). Data from all three replicates were pooled together. No cell-size gating was performed as assay allows for separation of extrinsic noise. To align fluorescence values of mClover and tdTomato on the same scale, each cell's fluorescence intensity was normalized to the mean expression level of that fluorophore for the population. Since Sox2 expression spans several orders of magnitude, cells were binned according to their total Sox 2 expression (mClover + tdTomato). Bins with fewer than 100 cells were discarded. Intrinsic noise $\left(\mathrm{CV}^{2}\right)$ of Sox 2 expression for each bin was calculated using the following formula:

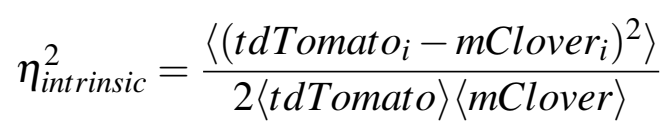

This value was then multiplied by the mean Sox 2 expression for each bin to obtain the Fano factor. Given that the number of cells in each bin differs and variance estimates are affected by sample size, we calculated $95 \%$ confidence intervals around the Fano factor for each bin through 
bootstrapping. Bin populations were resampled 10,000 times with replacement.

UV stress assay

$1 \times 10^{5}$ Nanog-GFP mESCs were seeded in each well of gelatin-coated, 12-well plates in 2i/LIF media. 24 hours following seeding, cultures were exposed to $3 \mathrm{~kJ}$ of $365 \mathrm{nM}$ light (Fotodyne UV Transilluminator 3-3000 with $15 \mathrm{~W}$ bulbs) for 15,30 or 60 minutes at room temperature in the dark. Control plates were left at room temperature in the dark for equivalent periods of time. Cells from UV-exposed and control plates were run unfixed and live on BD FACS Calibur cytometer $1,2,4,8$, and 12 hours post-exposure in replicate. Extrinsic noise filtering via cell-size gating was performed prior to calculation of Nanog Fano factor.

$\underline{\text { Live-cell time-lapse microscopy }}$

$1 \times 10^{5}$ Nanog-GFP mESCs were seeded into each well of a gelatin-coated, $35 \mathrm{~mm}$ Ibidi dish (quad-chambered, cat:80416) in 2i/LIF media. 24 hours following seeding, media was replenished with $2 \mathrm{i} / \mathrm{LIF}$ containing $10 \mu \mathrm{M}$ IdU or equivalent volume DMSO in replicate. Time-lapse imaging commenced immediately after addition of compounds with IdU- and DMSO- treated cells imaged in the same experiment (neighboring wells). Imaging was performed on a Zeiss Axio Observer Z1 microscope equipped with Yokogawa CSU-X1 spinning disk unit and a Cool-SNAP HQ2 14-bit camera (PhotoMetrics). 488nM laser line (50\% laser power, 500-ms excitation) was used for GFP imaging. Samples were kept in an enclosed stage that maintained humidified conditions at $37^{\circ} \mathrm{C}$ and $5 \% \mathrm{CO}_{2}$. Images were captured every 20 minutes for 24 hours. For each xy location, three z-planes were sampled at 4- $\mu$ m intervals. The objective used was 40x oil, 1.3 N.A.

Cell segmentation, tracking and GFP quantification were carried out using CellProfiler (95). Tracking of cells was manually verified. Segmented cells tracked for less than 4 hours were discarded. Cell division triggered the start of 2 new trajectories. After illumination correction and background subtraction, the mean GFP fluorescence intensity of a segmented cell was taken from each z-plane and averaged over the entire z-stack. For each trajectory, noise autocorrelation $\left(\tau_{1 / 2}\right)$ and noise magnitude (intrinsic- $\mathrm{CV}^{2}$ ) were calculated as previously described (52). Fluorescence trajectories were first detrended (normalized) by subtracting the population timedependent average fluorescence to isolate intrinsic noise. Distributions of noise frequency ranges $\left(F_{N}\right)$ were extracted from normalized autocorrelation functions (ACFs) of individual trajectories, where $F_{N}=\frac{1}{\tau_{1 / 2}}$. $\tau_{1 / 2}$ is the value of $\tau$ (lag time) where the normalized ACF reaches a value of 0.5.

$\underline{\text { Nucleoside analog screening }}$

14 nucleoside analogs (compound names and sources listed in Table S3) were resuspended in DMSO. $1 \times 10^{5}$ Nanog-GFP mESCs were seeded in gelatin-coated 12-well plates in 2i/LIF media. 24 hours after seeding, media was swapped with 2i/LIF containing $10 \mu \mathrm{M}$ of nucleoside analog or equivalent volume DMSO in replicate. After 24 hours of treatment, cells were run unfixed and 
live on BD LSRII cytometer. Extrinsic noise filtering via cell-size gating was performed prior to calculation of Nanog Fano factor. Fano factor for Nanog-GFP expression for each treatment was normalized to DMSO control.

Generation of stable CRISPRi Nanog-GFP mESC line

To stably integrate the CRISPRi machinery into the ROSA26 locus of Nanog-GFP mESCs, AAVS1 homology arms of the CRISPRi knockin construct (krab-dCas9-p2a-mCherry, Addgene:73497) were swapped with ROSA26 homology arms. The dox-inducible promoter of this construct was replaced with a constitutive CAGGS promoter and the kanamycin resistance cassette was replaced with puromycin resistance. Two million Nanog-GFP mESCs were nucleofected with the CRISPRi knockin construct and left to recover for 48 hours. Puromycin $(1 \mu \mathrm{g} / \mathrm{ml})$ selection was run until single colonies could be picked. Clonal CRISPRi Nanog-GFP mESC lines were assessed for mCherry expression and ability to knockdown Nanog. We selected the clone with the highest percentage of mCherry-positive cells.

CRISPRi gRNA design and cloning

gRNA sequences were were taken from the mCRISPRi-v2 library (91). gRNA oligos were annealed and cloned into the pU6-sgRNA EF1Alpha-puro-T2A-BFP lentiviral vector (Addgene:60955) using the BstXI/BlpI ligation strategy (91).

CRISPRi screening for genetic dependencies of noise enhancer

25 genes involved in nucleotide metabolism, DNA repair, and chromatin remodeling were screened for their potential role in noise enhancement from IdU. Three gRNAs were designed per gene (gene names and gRNA sequences listed in Table S4). Three non-targeting controls (scrambled gRNAs) were taken from the mCRISPRi-v2 library (91). Each gRNA expression plasmid was separately packaged into lentivirus in HEK293T cells as previously described (91). For each gRNA lentivirus, $1.5 \times 10^{5}$ CRISPRi Nanog-GFP mESCs were spinoculated with filtered viral supernatant for 90 minutes at $200 \mathrm{x}$ g in replicate. Following spinoculation, infected cells were seeded into gelatin-coated, 6-well plates in 2i/LIF media. 48 hours following seeding, media was swapped with $2 \mathrm{i} / \mathrm{LIF}$ supplemented with either $10 \mu \mathrm{M}$ IdU or equivalent volume DMSO. Consequently, for every knockdown there is a DMSO and IdU treatment group. After 24 hours of treatment, cells were run unfixed and live on a BD LSRII flow cytometer. To minimize technical variability, analysis was restricted to cells with homogeneous levels of dCas9-KRAB and gRNA expression through stringent gating on mCherry/BFP double-positive cells. Extrinsic noise filtering through cell-size gating was then applied. For each gRNA, Nanog Fano factor for the DMSO and IdU treatments were normalized to the Nanog Fano factor of the non-targeting controls treated with DMSO.

$\underline{\text { qPCR verification of CRISPRi knockdown }}$ 
To verify CRISPRi knockdown of Apex 1 and Tk1, each of the six gRNA-expression plasmids targeting these two genes along with a non-targeting control and empty vector were packaged into lentivirus. $1.5 \times 10^{5}$ CRISPRi Nanog-GFP mESCs were spinoculated with filtered viral supernatant for 90 minutes at $200 \mathrm{x}$ g in replicate. Following spinoculation, infected cells were seeded into gelatin-coated, 6-well plates in 2i/LIF media. 72 hours following seeding, $1 \times 10^{6}$ mCherry/BFP double-positive cells from each infected cell population were sorted on a FACSAria II. Total RNA was extracted using an RNeasy Mini Kit (QIAGEN cat:74104) and reverse-transcribed using a QuantiTect Reverse Transcription Kit (QIAGEN cat:205311). cDNA from each independent biological replicate was plated in triplicate and run on a 7900HT Fast Real-Time PCR System (Thermo) using designed primers (Table S5) and Fast SYBR Green Master Mix (Applied Biosystems, cat:4385612). Expression of GAPDH was used for normalization. Relative mRNA levels of Apex 1 and Tk1 were calculated by the $\Delta \Delta C_{t}$ method using the empty-vector populations as the control. All reported levels of repression are relative to the non-targeting control.

\section{Tk1 competition assay}

$1 \times 10^{5}$ Nanog-GFP mESCs were seeded in each well of gelatin-coated, 12 -well plates in 2i/LIF media. 24 hours following seeding, media was replaced with $2 \mathrm{i} / \mathrm{LIF}$ supplemented with $10 \mu \mathrm{M}$ IdU in combination with thymidine (Sigma cat:T1895) or uridine (Sigma cat:U3003) at concentrations ranging from 0 to $100 \mu \mathrm{M}$. Concentration combinations were done in triplicate. After 24 hours of treatment, cells were run unfixed and live on BD FACS Calibur cytometer. Extrinsic noise filtering via cell-size gating was performed prior to calculation of Nanog Fano factor.

\section{Biotinylated-trimethylpsoralen (bTMP) supercoiling assay}

$1 \times 10^{5}$ Nanog-GFP mESCs were seeded into each well of a gelatin-coated, $35 \mathrm{~mm}$ Ibidi dish (quad-chambered, cat:80416) in 2i/LIF media. 24 hours following seeding, media was replaced

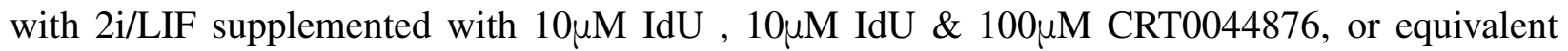
volume DMSO in replicate. After 24 hours of treatment, media was replaced with $2 \mathrm{i} / \mathrm{LIF}$ supplemented with $1 \mu \mathrm{M}$ aphidicolin for two hours. For control experiments, Nanog-GFP mESCs were cultured with or without $10 \mu \mathrm{M}$ IdU for 24 hours followed by treatment with $100 \mu \mathrm{M}$ bleomycin for one hour. Cells were then washed 1xDPBS and then permeabilized with $0.1 \%$ Tween-20 in DPBS for 15 minutes. Cells were then incubated with $0.3 \mathrm{mg} / \mathrm{ml}$ EZ-Link Psoarlen-PEG3-Biotin (Thermo cat:29986) for 15 minutes. Cultures were then exposed to 365nM light (AlphaImager HP with $15 \mathrm{~W}$ bulbs, ProteinSimple) for 15 minutes at room temperature. Cells were then washed 2xDPBS, fixed with cold $70 \%$ ethanol for 30 minutes at $4{ }^{\circ} \mathrm{C}$, and then washed $2 x D P B S$. Cells were then incubated with Alexa Fluor 594 Streptavidin (Thermo cat:S32356) for one hour at room temperature in the dark, washed 2xDPBS, and stained with DAPI for 10 minutes at room temperature in the dark. Cells were imaged in a buffer containing $50 \%$ glycerol (Thermo), $75 \mu \mathrm{g} / \mathrm{mL}$ glucose oxidase (Sigma Aldrich), $520 \mu \mathrm{g} / \mathrm{mL}$ catalase (Sigma Aldrich), and $0.5 \mathrm{mg} / \mathrm{mL}$ Trolox (Sigma Aldrich). Images were taken on a Zeiss Axio Observer Z1 microscope equipped with a Yokogawa CSU-X1 spinning disk unit and 63x/1.4 oil objective. Approximately 20 xy locations 
were randomly selected for each condition. For each xy location, three z-planes were sampled at 4- $\mu \mathrm{m}$ intervals. Nuclear segmentation using DAPI signal and quantification of psoralen staining intensity were carried out using CellProfiler. After illumination correction and background subtraction, the mean psoralen fluorescence intensity of a segmented nucleus was taken from each z-plane and averaged over the entire z-stack.

96 dose combination for testing of noise phase space

Compound plates containing 96 concentration combinations of IdU, BrdU, or HmU with CRT0044876 were prepared by the Gladstone Assay Development and Drug Discovery Core using an Agilent Bravo liquid handling system. All wells contained equivalent volumes of DMSO. Compound mixtures were suspended in $200 \mu \mathrm{L}$ of $2 \mathrm{i} / \mathrm{LIF}$ media. $1 \times 10^{4}$ Nanog-GFP mESCs were seeded into each well of a gelatin-coated, 96-well dish in $200 \mu \mathrm{L}$ of $2 \mathrm{i} / \mathrm{LIF}$ media. 24 hours after seeding, 100 $\mu \mathrm{L}$ of media was removed from each well and $100 \mu \mathrm{L}$ of compound-containing $2 \mathrm{i} / \mathrm{LIF}$ was added in replicate. Layout and final concentrations of treatments are listed in Table S6. IdU and BrdU concentrations ranged from 0 to $50 \mu \mathrm{M}$ while $\mathrm{HmU}$ concentrations ranged from 0 to $10 \mu \mathrm{M}$. CRT0044876 ranged from 0 to $150 \mu \mathrm{M}$. After 24 hours of treatment, cells were detached using TrypLE and plates were run on BD LSRFortessa high-throughput system. After extrinsic noise filtering via cell-size gating, Nanog mean and Fano factor for each treatment were normalized to DMSO control well. Reported fold changes in mean and Fano factor are the average of two replicates.

Estimation of promoter toggling kinetics from scRNA-seq data

Gene expression data from the scRNA-seq dataset were fit to the 2-state model using the D3E algorithm, allowing for estimation of $k_{O N}, k_{O F F}, a n d k_{t x}$ in proportion to the rate of mRNA degradation which is the lone parameter that is not estimable from this dataset alone (83). Parameter estimation was conducted using the methods of moments approach with the normalise and removeZeros options. Analysis was run for the 945 genes classified as highly variable according to the BASiCS algorithm. The Cramer-von Mises test was used for goodness-of-fit testing. Values of $k_{\text {decay }}$ were then retrieved from an existing dataset of mRNA degradation rates in mESCs (92), with the assumption that degradation rates are unchanged between DMSO and IdU conditions. Parameter estimates were then verified against experimental values of mean mRNA counts using the following relationship: $\langle R N A\rangle=\frac{k_{O N}}{k_{O N}+k_{O F F}} \cdot \frac{k_{R N A}}{k_{\text {decay }}}$. Genes whose predicted mean was within $10 \%$ of experimental value were used for downstream analysis. 314 genes passed this filtering process based on availability of mRNA degradation rates and alignment of parameter estimates with expected mean mRNA counts.

Mathematical modeling and simulations

Detailed descriptions of model development, parameterization, evaluation, and sensitivity analysis can be found in the supplementary text along with details of Gillespie simulations. 
Estimation of cellular developmental potential and Waddington Landscape reconstruction

The HopLand algorithm (continuous Hopfield network) was used to create a predictive model of gene-gene interactions from scRNA-seq data (86). As input, raw count data for a total of 800 randomly chosen cells (400 from DMSO and 400 from IdU treatment groups) were used. Each neuron of the network corresponds to a gene. Genes whose variance fell within in the top $10 \%$ were used for construction of a neural network resulting in 512 nodes. The weight matrix describing pair-wise interactions between nodes was initialized using the gene-gene Pearson correlation matrix. A Gaussian process latent variable model (GP-LVM) was used for dimensionality reduction to create a 2-D map of cell clustering ( $\mathrm{x}$ and y coordinates on Waddington Landscape). Energy values ( $\mathrm{z}$ coordinate on landscape) were calculated using the Lyapunov function which is a measure of stability. Lower energy values indicate greater proximity to an equilibrium point (attractor state) and thus less developmental potential.

Cellular reprogramming assays

Two cellular reprogramming systems were tested in this study: (1) Nanog-GFP secondary mouse embryonic fibroblasts (MEFs) harboring stably integrated, doxycycline-inducible cassettes for Oct4, Sox2, and Klf4. GFP is expressed from the endogenous Nanog locus. (2) Oct4-GFP primary MEFs that express GFP from the endogenous Oct4 locus.

Secondary MEFs were seeded onto gelatin-coated, 12-well plates at a density of $10,000 \mathrm{cells} / \mathrm{cm}^{2}$ in MEF medium (DMEM supplemented with $10 \%$ FBS and $0.1 \mathrm{mM}$ non-essential amino acid, and 2mM Glutamax). 24 hours after seeding, wells were washed with DPBS and media was switched to ESC media (knockout DMEM, 10\% FBS, 10\% KSR, 2mM Glutamax, 0.1mM non-essential amino acid, $0.1 \mathrm{mM}$ 2-mercaptoethanol, $10^{3}$ units $/ \mathrm{ml}$ leukemia inhibitory factor) supplemented with $1 \mu \mathrm{g} / \mathrm{ml}$ doxycycline. Additionally, IdU ( $1 \mathrm{uM}$ or $4 \mathrm{uM}$ ) or equivalent volume DMSO (Day 0 ) were added to media. 48 hours after the start of IdU treatment, wells were washed with DPBS and media was replaced with ESC media supplemented with $1 \mu \mathrm{g} / \mathrm{ml}$ doxycycline alone. Media was refreshed every other day until day 10 of reprogramming. Alkaline phosphatase staining was performed according to manufacturer's instructions using the Alkaline Phosphatase Detected Kit (Millipore). For flow cytometric analysis of Nanog-GFP expression, cells were dissociated with TrypLE and run unfixed on BD FACS Calibur cytometer.

Oct4-GFP primary MEFs were transduced with lentiviral vectors encoding Oct4, Sox2, Klf4, and c-Myc. Lentiviruses encoding these factors were individually packaged in PLAT-E cells (ATCC) using pMX-based vectors. 48 hours after transfection of lentiviral vectors, viral supernatant was collected and filtered. For infection, Oct4-GFP primary MEFs were seeded on gelatincoated, 6-well plates at a density of 10,000 cells $/ \mathrm{cm}^{2}$ in MEF medium 24 hours prior to transduction (Day -2). Oct4, Sox2, Klf4, and c-Myc viruses were mixed in equal volume along with 5 $\mathrm{g} / \mathrm{ml}$ polybrene and incubated with primary MEFs for 24 hours in MEF medium (Day -1). Following infection, wells were washed with ESC media and cells were incubated with ESC media supple- 
mented with $10 \mu \mathrm{M}$ Forskolin, $1 \mathrm{mM}$ Valproic Acid, $4 \mu \mathrm{M}$ IdU or equivalent volume DMSO (Day $0)$. ESC media was refreshed every other day. IdU supplementation was discontinued after 48 hours while Forskolin and Valproic Acid were kept in media continuously. Oct4-GFP(+) colonies were counted on days 8,10 and 12 .

$\underline{\text { Bulk RNA-seq of secondary MEFs undergoing reprogramming }}$

Secondary MEFs were seeded onto gelatin-coated, 6-well plates at a density of $10,000 \mathrm{cells} / \mathrm{cm}^{2}$ in MEF medium. For each timepoint (2- and 5-day), 4 wells were seeded ( 2 replicates for standard reprogramming and 2 replicates for IdU-assisted reprogramming). 24 hours after seeding, wells were washed with DPBS and media was switched to ESC media supplemented with $1 \mu \mathrm{g} / \mathrm{ml}$ doxycycline. Additionally, $4 \mu \mathrm{M}$ IdU or equivalent volume DMSO (Day 0) were added to media. 48 hours after the start of reprogramming, cells for the 2-day timepoint in DMSO and IdU conditions were dissociated with TrypLE, pelleted, and snap frozen with liquid nitrogen. Media in the wells for the 5-day timepoint was refreshed with ESC media supplemented with $1 \mu \mathrm{g} / \mathrm{ml}$ doxycycline alone. This was repeated on day 4 . On day 5 , remaining cells were dissociated and frozen identically to that of the 2-day timepoint.

RNA was extracted from each cell pellet using a RNeasy minikit (Qiagen) according to manufacturer's instructions. A total of 8 cDNA libraries were prepared with an NEBNext Ultra II RNA Library Prep kit (NEB, cat:E7770S) and sequenced with an Illumina HiSeq4000. Sequencing yielded a median of $\approx 50$ million single-end reads per library. Read quality was checked via FASTQC. Reads were aligned to the mm10 reference genome using TopHat with default parameters. Transcript level quantification was performed using Cufflinks with default parameters. 


\section{Supplementary Text}

\section{Overview of tested models}

To delineate how Apex 1 alters transcriptional dynamics of Nanog gene expression in a way that increases noise without changing mean, we developed a series of models that allow for Apex1 interaction at different stages of the transcription process. Through stochastic simulation of these models and comparison to experimental data, the aim is to develop greater mechanistic insight into what stages of the transcription process Apex 1 affects. The tested models listed in supplementary figure 20 are adapted from the two-state random telegraph model. We assume in each of the models that IdU incorporation into the genome leads to recruitment of Apex1 ( $k_{\text {incorpo }}$ ). The resulting interaction results in a transcriptionally non-productive state. Unbinding of Apex1 is triggered by completion of repair $\left(k_{\text {repair }}\right)$.

\section{Detailed mathematics and derivation of parameter constraints}

We derive here some relations, at equilibrium, between the kinetic rates of the diverse models. These relationships are then used to constrain the parameter phase space for a given set of data.

\subsection{Model 0}

This is the null model and consists of only the canonical two-state random-telegraph. This model is used as a null hypothesis, in particular for log-likelihood and AIC-based model selection.

\subsection{Model 1}

We assume that IdU incorporation and subsequent interaction of Apex 1 with the chromatin, occurs only in the OFF state of the promoter. Biologically, this may occur if control mechanisms inhibit DNA repair during active transcription.

The differential equations governing the gene fractions in the different states and the mRNA counts are as follows: 


$$
\left\{\begin{array}{l}
\frac{d O F F}{d t}=k_{O N} \cdot O N+k_{\text {repair }} \cdot O F F^{*}-\left(k_{\text {incorpo }}+k_{O N}\right) \cdot O F F \\
\frac{d O N}{d t}=k_{O N} \cdot O F F-k_{O F F} \cdot O N \\
\frac{d O F F^{*}}{d t}=k_{\text {incorpo }} \cdot O F F-k_{\text {repair }} \cdot O F F^{*} \\
\frac{d R N A}{d t}=k_{R N A} \cdot O N-k_{\text {decay }} \cdot R N A
\end{array}\right.
$$

Using the fact that:

$$
\frac{K_{O N}}{K_{O N}+K_{O F F}} \equiv \frac{O N}{O N+O F F+O F F^{*}}
$$

we derive the following constraint between $k_{\text {repair }}$ and $k_{\text {incorpo }}$, where $K_{O F F}$ represents the transition rate to the macroscopic OFF state consisting of the OFF $\left(k_{O F F}\right)$ and $\mathrm{OFF}^{*}\left(k_{\text {incorpo }}\right)$ states in Model 1:

$$
k_{\text {repair }}=\frac{K_{O N}+K_{O F F}}{K_{O N}} \cdot \frac{k_{O N}}{k_{O N}+k_{O F F}+k_{\text {incorpo }}}-k_{\text {incorpo }}
$$

\subsection{Model 2}

In this model, Apex1 can interact with chromatin in both the $\mathrm{ON}$ and OFF states of the promoter. If Apex1 interacts with the chromatin in the ON state, this leads to a turning off of the system. Molecularly this may be seen as a strong inhibitory effect mediated by Apex1: the interaction may recruit chromatin modifiers (e.g. histone deacetylases, histone methyltransferases) that silence gene expression. In the same way, stalled polymerases, at both the promoter proximal region and further in the gene, may unbind DNA.

This model can be described by the following set of ODEs: 


$$
\left\{\begin{array}{l}
\frac{d O F F}{d t}=k_{O F F} \cdot O N+k_{\text {repair }} \cdot O F F^{*}-\left(k_{\text {incorpo }}+k_{O N}\right) \cdot O F F \\
\frac{d O N}{d t}=k_{O N} \cdot O F F-\left(k_{O F F}+k_{\text {incorpo }}\right) \cdot O N \\
\frac{d O F F^{*}}{d t}=k_{\text {incorpo }} \cdot O F F+k_{\text {incorpo }} \cdot O N-k_{\text {repair }} \cdot O F F^{*} \\
\frac{d R N A}{d t}=k_{R N A} \cdot O N-k_{\text {decay }} \cdot R N A
\end{array}\right.
$$

Using equation (6) we derive the following constraint between $k_{\text {repair }}$ and $k_{\text {incorpo }}$ :

$$
\frac{k_{\text {incorpo }}}{k_{\text {repair }}}=\frac{K_{O F F}}{K_{O N}} \cdot \frac{k_{O N}}{k_{O F F}}-1
$$

\subsection{Model 3}

Here we assume that Apex1 can still interact in the ON state but that does not alter the "primed" characteristic of the gene expression system. The system is thus in a transcriptionally non-productive ON* state. In other words transcription can not be achieved when Apex1 interacts with the chromatin but the transcriptionally permissive chromatin and molecular context is not altered: primed polymerases remain, transcription enhancing epigenetic marks are not erased, etc.

This model can be described by the following set of ODEs:

$$
\left\{\begin{array}{l}
\frac{d O F F}{d t}=k_{O F F} \cdot O N+k_{\text {repair }} \cdot O F F^{*}-\left(k_{\text {incorpo }}+k_{O N}\right) \cdot O F F \\
\frac{d O N}{d t}=k_{O N} \cdot O F F+k_{\text {repair }} \cdot O N^{*}-\left(k_{O F F}+k_{\text {incorpo }}\right) \cdot O N \\
\frac{d O F F^{*}}{d t}=k_{\text {incorpo }} \cdot O F F-k_{\text {repair }} \cdot O F F^{*} \\
\frac{d O N^{*}}{d t}=k_{\text {incorpo }} \cdot O N-k_{\text {repair }} \cdot O N^{*} \\
\frac{d R N A}{d t}=k_{R N A} \cdot O N-k_{\text {decay }} \cdot R N A
\end{array}\right.
$$


We can thus derive the following constraint:

$$
\frac{k_{\text {incorpo }}}{k_{\text {repair }}}=\frac{1}{k_{O N}+k_{O F F}} \cdot\left[k_{O N} \cdot \frac{K_{O F F}}{K_{O N}}-k_{O F F}\right]
$$

The details of this derivation are described below for Model 4.

\subsection{Model 4}

Model 4 is based on Model 3, but an amplification step was added. When the system transitions from $\mathrm{ON}^{*}$ to $\mathrm{ON}$, the basal transcription rate $\left(k_{R N A}^{0}\right)$ increased by a multiplicative factor: $k_{R N A 2}=\operatorname{coop} \cdot k_{R N A}^{0}$. Thus we assume that there is molecular memory of the repair event. This may be rooted in a modification of supercoiling, polymerase accumulation, or chromatin remodeling.

This model can be described by the following set of ODEs :

$$
\left\{\begin{array}{l}
\frac{d O F F}{d t}=k_{O F F} \cdot O N+k_{\text {repair }} \cdot O F F^{*}-\left(k_{\text {incorpo }}+k_{O N}\right) \cdot O F F \\
\frac{d O N}{d t}=k_{O N} \cdot O F F+k_{\text {repair }} \cdot O N^{*}-\left(k_{\text {incorpo }}+k_{O F F}\right) \cdot O N \\
\frac{d O F F^{*}}{d t}=k_{\text {incorpo }} \cdot O F F-k_{\text {repair }} \cdot O F F^{*} \\
\frac{d O N^{*}}{d t}=k_{\text {incorpo }} \cdot O N-k_{\text {repair }} \cdot O N^{*} \\
\frac{d R N A}{d t}=\left\langle k_{R N A}\right\rangle \cdot O N-k_{\text {decay }} \cdot R N A
\end{array}\right.
$$

Where $k_{\text {incorpo }}=[I d U] \cdot k_{\text {incorpo }}^{0}$. We assume that IdU is in excess and thus $[\mathrm{IdU}]$ remains constant. At equilibrium :

$$
\frac{d O F F}{d t}=\frac{d O N}{d t}=\frac{d O F F^{*}}{d t}=\frac{d O N^{*}}{d t}=\frac{d R N A}{d t}=0
$$




$$
\Longrightarrow\left\{\begin{array}{l}
O F F_{e q}=\frac{k_{O F F}}{k_{O N}} \cdot O N_{e q} \\
O N_{e q}=\frac{k_{O N}}{k_{O F F}} \cdot O F F_{e q} \\
O F F_{e q}^{*}=\frac{k_{\text {incorpo }}}{k_{\text {repair }}} \cdot O F F_{e q} \\
O N_{e q}^{*}=\frac{k_{\text {incorpo }}}{k_{\text {repair }}} \cdot O N_{e q} \\
R N A_{e q}=\frac{\left\langle k_{R N A}\right\rangle}{k_{\text {decay }}} \cdot O N_{e q}
\end{array}\right.
$$

By definition:

$$
\frac{K_{O N}}{K_{O N}+K_{O F F}} \equiv \frac{O N}{O N+O F F+O N^{*}+O F F^{*}}
$$

where $K_{O F F}$ represents the transition rate to the macroscopic OFF state (defined by no transcription) consisting of the $\mathrm{OFF}, \mathrm{OFF}^{*}$, and $\mathrm{ON}^{*}$ states. Using the set of equations in (10) and (11) we obtain:

$$
\frac{K_{O N}}{K_{O N}+K_{O F F}}=\frac{k_{\text {repair }}}{k_{\text {repair }}+k_{\text {incorpo }}} \cdot \frac{k_{O N}}{k_{O N}+k_{O F F}}
$$

Equation (12) can be seen as :

$$
P\left(\text { ON }_{\text {macro }}\right)=P(\text { "Repaired state" }) \cdot P\left(\text { ON }_{\text {micro }}\right)
$$

Because $P\left(O N_{\text {macro }}\right) \equiv P\left(\right.$ "Repaired state" $\left.\cap O N_{\text {micro }}\right)$ we can deduce that "Repaired state" and $O N_{\text {micro }}$ are independent probabilistic events.

Equation (12) can be rewritten as:

$$
\frac{K_{O N}}{K_{O F F}}=\frac{k_{\text {repair }} \cdot k_{O N}}{k_{O F F} \cdot\left(k_{\text {repair }}+k_{\text {incorpo }}\right)+k_{O N} \cdot k_{\text {incorpo }}}
$$


Which gives us:

$$
\frac{k_{\text {incorpo }}}{k_{\text {repair }}}=\frac{1}{k_{O N}+k_{O F F}} \cdot\left[k_{O N} \cdot \frac{K_{O F F}}{K_{O N}}-k_{O F F}\right]
$$

Let us define $\left\langle k_{R N A}\right\rangle$ and $k_{R N A}^{0}$ as the mean transcription rate in the presence of IdU and the transcription rate in the control condition (DMSO), respectively. By construction of the model:

$$
\left\langle k_{R N A}\right\rangle=P(O N \mid O F F) \cdot k_{R N A}^{0}+P\left(O N \mid O N^{*}\right) \cdot \operatorname{coop} \cdot k_{R N A}^{0}
$$

The coop term represents the amplification of the transcription rate following completion of repair. $P(O N \mid O F F)$ and $P\left(O N \mid O N^{*}\right)$ represent the probability that the gene transitioned to the ON state from the $\mathrm{OFF}$ and $\mathrm{ON}^{*}$ states respectively.

Or :

$$
\left\{\begin{array}{l}
P(O N \mid O F F)=\frac{k_{O N} \cdot O F F}{k_{O N} \cdot O F F+k_{\text {repair }} \cdot O N^{*}} \\
P\left(O N \mid O N^{*}\right)=\frac{k_{\text {repair }} \cdot O N^{*}}{k_{O N} \cdot O F F+k_{\text {repair }} \cdot O N^{*}}
\end{array}\right.
$$

Combining equations (14) and (16) we get:

$$
\left\{\begin{array}{l}
P(O N \mid O F F)=\frac{k_{O F F}}{k_{O F F}+k_{\text {incorpo }}} \\
P\left(O N \mid O N^{*}\right)=\frac{k_{\text {incorpo }}}{k_{O F F}+k_{\text {incorpo }}}
\end{array}\right.
$$

Rewriting (15) using (17) we obtain :

$$
\begin{gathered}
\frac{\left\langle k_{R N A}\right\rangle}{k_{R N A}^{0}}=\frac{1}{k_{O F F}+k_{\text {incorpo }}} \cdot\left(k_{\text {OFF }}+\text { coop } \cdot k_{\text {incorpo }}\right) \\
\Longrightarrow \text { coop }=\frac{k_{O F F}+k_{\text {incorpo }}}{k_{\text {incorpo }}} \cdot \frac{\left\langle k_{R N A}\right\rangle}{k_{R N A}^{0}}-\frac{k_{O F F}}{k_{\text {incorpo }}}
\end{gathered}
$$

Equation (19) can be rewritten to explicitly take into account [IdU] :

$$
\text { coop }=\frac{k_{O F F}+k_{\text {incorpo }}^{0} \cdot[I d U]}{k_{\text {incorpo }}^{0} \cdot[I d U]} \cdot \frac{\left\langle k_{R N A}\right\rangle}{k_{R N A}^{0}}-\frac{k_{O F F}}{k_{\text {incorpo }}^{0} \cdot[I d U]}
$$




\subsection{Model 5}

In this last model we make the same assumptions as in model 4 except that Apex 1 interaction with chromatin occurs only in the ON state. This does not imply that IdU incorporation and subsequent Apex 1 interaction only occurs in the ON state. Our assumption supposes that the interaction of Apex 1 in the OFF state is negligible quantitatively speaking compared to that in the ON state.

The ODEs describing this model are as follows:

$$
\left\{\begin{array}{l}
\frac{d O F F}{d t}=k_{O F F} \cdot O N-k_{O N} \cdot O F F \\
\frac{d O N}{d t}=k_{O N} \cdot O F F+k_{\text {repair }} \cdot O N^{*}-\left(k_{\text {incorpo }}+k_{O F F}\right) \cdot O N \\
\frac{d O N^{*}}{d t}=k_{\text {incorpo }} \cdot O N-k_{\text {repair }} \cdot O N^{*} \\
\frac{d R N A}{d t}=\left\langle k_{R N A}\right\rangle \cdot O N-k_{\text {decay }} \cdot R N A
\end{array}\right.
$$

The coop expression remains unchanged from Model 4 but the ratio between $k_{\text {repair }}$ and $k_{\text {incorpo }}$ changes as follows:

$$
\frac{K_{O N}}{K_{O F F}} \equiv \frac{O N}{O F F+O N^{*}}
$$

Thus we obtain:

$$
\frac{K_{O N}}{K_{O F F}}=\frac{k_{\text {repair }} \cdot k_{O N}}{k_{O F F} \cdot k_{\text {repair }}+k_{\text {incorpo }} \cdot k_{O N}}
$$

And :

$$
\frac{k_{\text {incorpo }}}{k_{\text {repair }}}=\frac{K_{O F F}}{K_{O N}}-\frac{k_{O F F}}{k_{O N}}
$$




\section{Chemical Master Equation}

For all models we constructed an associated stochastic scheme. As an example, Model 5 can be rewritten using the following scheme:

$$
\left\{\begin{array}{l}
\text { OF } F \stackrel{k_{O N}}{\longrightarrow} \text { ON } \\
O N_{1} \stackrel{k_{\text {OFF }}}{\longrightarrow} \text { OFF } \\
O N_{1} \stackrel{k_{R N A 1}}{\longrightarrow} R N A \\
O N_{1} \stackrel{k_{\text {incorpo }}}{\longrightarrow} O N^{*} \\
O N^{*} \stackrel{k_{\text {repair }}}{\longrightarrow} O N_{2} \\
O N_{2} \stackrel{k_{\text {incorpo }}}{\longrightarrow} O N^{*} \\
O N_{2} \stackrel{k_{\text {OFF }}}{\longrightarrow} \text { OFF } \\
O N_{2} \stackrel{k_{R N A 2}}{\longrightarrow} R N A \\
R N A \stackrel{k_{\text {decay }}}{\longrightarrow} \varnothing
\end{array}\right.
$$

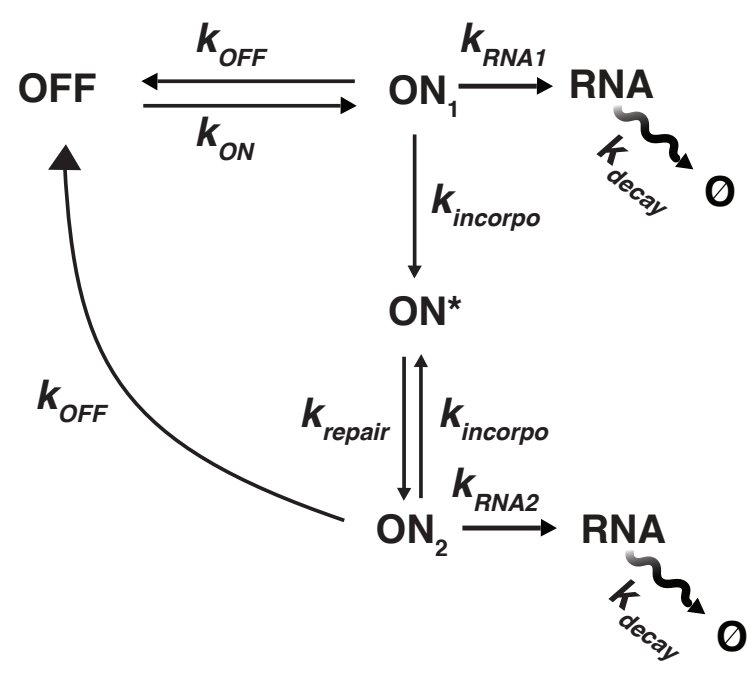

$\mathrm{ON}_{2}$ represents the repaired state of the gene, which results in a higher transcription rate $\left(k_{R N A 2}\right)$. Using this scheme, we can construct the chemical master equation (CME) describing the time dependent distributions of mRNA copy number:

$$
\frac{d \mathbf{P}(m, t)}{d t}=\mathbf{A} \cdot \mathbf{P}(m, t)+\boldsymbol{\delta}(\mathbf{E}-\mathbf{I})[m \mathbf{P}(m, t)]+\Delta\left(\mathbf{E}^{-1}-\mathbf{I}\right)[\mathbf{P}(m, t)]
$$


Where $\mathbf{A}, \Delta$, and $\delta$ are the transition, transcription, and degradation matrices respectively:

$$
\begin{gathered}
\mathbf{A}=\left[\begin{array}{cccc}
-k_{O N} & 0 & -k_{O F F} & -k_{O F F} \\
0 & -k_{\text {repair }} & k_{\text {incorpo }} & k_{\text {incorpo }} \\
k_{O N} & 0 & -\left(k_{O F F}+k_{\text {incorpo }}\right) & 0 \\
0 & k_{\text {repair }} & 0 & -\left(k_{\text {incorpo }}+k_{O F F}\right)
\end{array}\right] \\
\Delta=\left[\begin{array}{cccc}
0 & 0 & 0 & 0 \\
0 & 0 & 0 & 0 \\
0 & 0 & k_{R N A 1} & 0 \\
0 & 0 & 0 & k_{R N A 2}
\end{array}\right] \\
\delta=\left[\begin{array}{cccc}
k_{\text {decay }} & 0 & 0 & 0 \\
0 & k_{\text {decay }} & 0 & 0 \\
0 & 0 & k_{\text {decay }} & 0 \\
0 & 0 & 0 & k_{\text {decay }}
\end{array}\right]
\end{gathered}
$$

$\mathbf{P}(m, t)$ is a four-element column vector consisting of the time-dependent mRNA probability distributions while in the $O F F, O N^{*}, O N_{1}$, and $O N_{2}$ states respectively. $\mathbf{E}$ and $\mathbf{E}^{-1}$ are the forward and backward shift operators while $\mathbf{I}$ is the identity matrix.

At steady-state, the mRNA probability distribution can be reconstructed as a sum of the binomial moments (93)

$$
P(m)=\sum_{k \geq m}(-1)^{m-k}\left(\begin{array}{c}
k \\
m
\end{array}\right) b_{k}, m=0,1,2, \ldots
$$

Where $b_{k}$ is the $k^{\text {th }}$ binomial moment of the distribution given by:

$$
b_{k}=\frac{1}{\prod_{i=1}^{k} \operatorname{det}(i \delta-\mathbf{A})} \cdot \prod_{i=k}^{1}\left[\mathbf{u}_{N}(i \delta-\mathbf{A})^{*} \Delta\right] \cdot \mathbf{b}_{0}, k=1,2, \ldots
$$

where $\mathbf{u}_{N}=[1,1,1,1] .(i \delta-\mathbf{A})^{*}$ and $\operatorname{det}(i \delta-\mathbf{A})$ are the adjugate and the determinant of matrix $(i \delta-\mathbf{A})$ respectively. $b_{1}$ is equivalent to the mean mRNA abundance of the system at equilibrium. 
$\mathbf{b}_{0}$ is the corresponding eigenvector for the zero eigenvalue of $\mathbf{A}$. The 4 elements of $\mathbf{b}_{0}$ therefore represent the fraction of time spent in the $O F F, O N^{*}, O N_{1}$, and $O N_{2}$ states at equilibrium. The $n^{t h}$ component of $\mathbf{b}_{0}$ is given by:

$$
b_{0}^{(n)}=\prod_{i=1}^{3} \frac{\beta_{i}^{(n)}}{\alpha_{i}}, 1 \leq n \leq 4
$$

where $\alpha_{1}, \alpha_{2}, \alpha_{3}$ are the three non-zero eigenvalues of $\mathbf{A} . \beta_{1}^{(n)}, \beta_{2}^{(n)}, \beta_{3}^{(n)}$ are the three eigenvalues of the sub-matrix $\mathbf{M}_{n}$ which is constructed by removing the $n^{\text {th }}$ row and $n^{\text {th }}$ column of $\mathbf{A}$.

The Fano factor for mRNA counts at equilibrium is then given by:

$$
F F=\frac{2 b_{2}+b_{1}-b_{1}^{2}}{b_{1}}
$$

An exact simulation of such a stochastic process is given by the Gillespie algorithm. We implemented the algorithm using a homemade script in Julia 1.1.1. For each model, 1500 simulations were run for a virtual duration of $200 \mathrm{~h}$. Since the time is not discrete we used a "parsing" algorithm, based on recursive binary search, to align all the traces on a common time scale of 2000 intervals. For each interval of the discretized time we computed the average and variance of the number of mRNAs, taking into account all traces. The analytic relationships described above are used to verify the inferred kinetic rates from stochastic simulations.

\section{Estimation of model parameters from experimental data}

From the smRNA FISH data, we can infer the macroscopic kinetic rates (Table S2). The kinetic rates computed in the control condition (DMSO in Table S2) are at the basis of the simulations and have to be considered as constant for all the models and associated results.

Use of these macroscopic rates along with the relationships developed in section 2 results in one remaining degree of freedom for our models: $k_{\text {repair }}$ (or $k_{\text {incorpo }}$ for Model 1 ).

The confrontation between experimental data and the results of stochastic simulations for each of the models should allow us to define the consistency of our models and thus gain mechanistic insight into how Apex1 affects transcriptional dynamics. 


\section{Model selection: Comparison of simulation results to exper- imental data}

\subsection{Information theory-based approach: MLE and Akaike's criterion}

\subsubsection{Workflow}

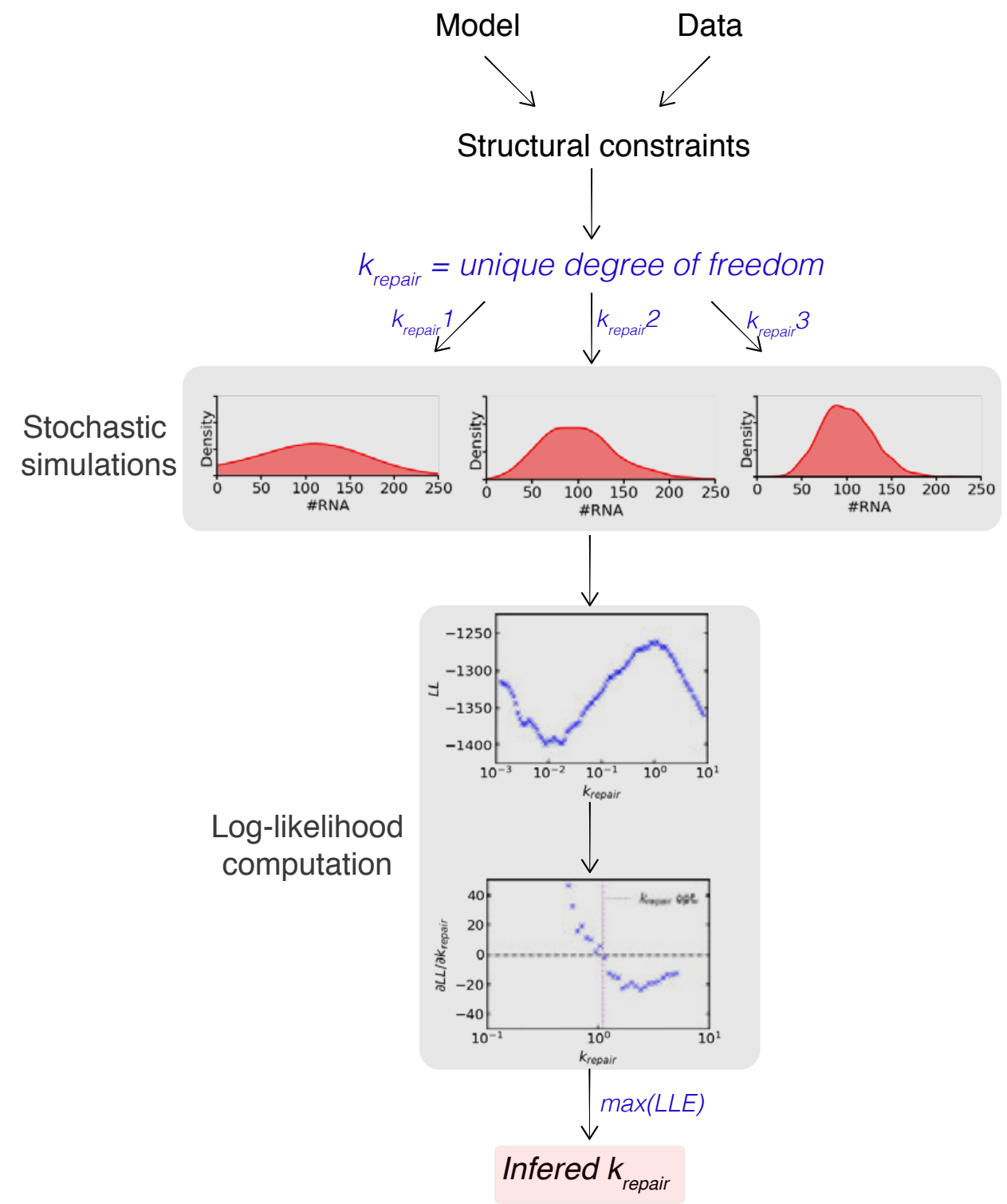




\subsubsection{Method of Log-likelihood and AIC computation}

For each of the 500 chosen values (logarithmically spaced) of our unique degree of freedom $\left(k_{\text {repair }}\right)$, we ran 50,000 simulations for a total duration of $200 \mathrm{~h}$. Only the last point of each simulation was stored. Then the steady-state distribution of RNA was computed using the same binning as in the experimental data. The computation of the log-likelihood is as follows:

Let $\mathbf{X}$ be the vector of $n$ empirical observations and let $\mathscr{X}_{\Delta}:\left\{x^{\Delta}, x^{\Delta} \in[x, x+\Delta]\right\}$, where $\Delta$ is the size of a bin and $\mathscr{X}_{\Delta}$ is the set of bins. We define $\hat{p}\left(x^{\Delta}\right)$ as the probability of observing an experimental value within a particular bin $\left(x^{\Delta}\right) . P\left(x^{\Delta}\right)$ is the probability of a given $x^{\Delta} \in[x, x+\Delta]$ for a given model. The likelihood function $\mathscr{L}$ is defined as :

$$
\mathscr{L} \equiv \prod_{x_{i} \in \mathbf{X}} P\left(x_{i}, x_{i} \in[x, x+\Delta]\right)=\prod_{x^{\Delta} \in \mathscr{X}_{\Delta}} P\left(x^{\Delta}\right)^{n \cdot \hat{p}\left(x^{\Delta}\right)}
$$

The log-likelihood is then given as:

$$
\log (\mathscr{L})=\sum_{x^{\Delta} \in \mathscr{X}_{\Delta}} n \hat{p}\left(x^{\Delta}\right) \cdot \log \left(P\left(x^{\Delta}\right)\right)
$$

$\mathscr{L}$ is a function of $k_{\text {repair. }}$ We then try to find the value of $k_{\text {repair }}$ that maximizes $\log (\mathscr{L})$ :

$$
\hat{k}_{\text {repair }, L L E}=\operatorname{argmax}_{k}(\log (\mathscr{L}(k)))
$$

With $k_{\text {repair }} \in\left[10^{-4}, 10\right]$, by assumption. After computing $\log \left(\mathscr{L}^{(k)}\right)$ for each of the 500 values of $k_{\text {repair }}$, we apply a smoothing (moving average) to the data, take the derivative, smooth the derivative, find the two points on each side of the abscissa, and then interpolate the point for which the derivative is equal to zero using a linear interpolation. The maximum $\log (\mathscr{L})$ is computed after the first smoothing. Then, we compute the macroscopic behavior of the system using the inferred value of $k_{\text {repair. }}$

The model selection is based on the $A I C$ and the resulting measures $\Delta_{i} A I C$ and $w_{i}(94)$. Because $\hat{k}_{\text {repair,LLE }}$ is dependent on the empirical distribution, we can assume that it is only an estimate of the true value, which we'll call $k_{\text {repair. }}^{0}$. Thus we want to reduce as much as possible the distance between $\hat{k}_{\text {repair,LLE }}$ and $k_{\text {repair }}^{0}$. This optimization problem allows us to derive the so-called Akaike's information criterion (AIC) as a measure to compare models. AIC is an estimate of the expected relative distance between the fitted model and the unknown true mechanism that actually generated the observed data:

$$
A I C=-2 \log \left(\mathscr{L}\left(\hat{k}_{\text {repair }, L L E}\right)\right)+2 K
$$

with $K$ the number of degrees of freedom of the system. 


\subsubsection{Results}

As shown in Figures S20A-B, Model 5 is selected on the basis of AIC. Model 4 is second-best. Model 5 qualitatively and quantitatively matches experimental data with its inferred $k_{\text {repair }}$.

\subsubsection{Parameter identifiability}

We used bootstrapping to assess the quality of the inference of $k_{\text {repair }}$ for Model 5 using MLE estimator. This method allows the computation of the confidence/credible interval (CI) for $k_{\text {repair }}$, and, in the framework of Bayesianism, a posterior distribution $P\left(\right.$ Data $\left.\mid k_{\text {repair }}\right)$ using a non-informative prior (94). The distribution of the MLE is peaked around a particular value, suggesting parameter identifiability (Figure S20C).

\subsection{APE-based approach}

\subsubsection{Workflow}

The second approach for model selection that we employed involves comparison of the macroscopic behavior of simulation results to experimental data. After deriving constraints on the phase space spanned by $k_{\text {repair }}$ we simulate each of the models over a range of $k_{\text {repair }}$ values. From these stochastic simulations, we then extract the macroscopic behavior of the system for a given model. We computed the mean number of mRNA and the Fano factor at equilibrium, on the last 100 time points of the traces. $k_{o f f}$ and $k_{o n}$ were computed using a non-linear curve fitting assuming exponentially distributed residence times (Poisson process). Both $v_{o n}$ and the burst size were computed using their basic definitions. The density of mRNA population was computed using the last points of the simulations. A classic kernel density was used to represent the data.

For each model, we then infer the best value of $k_{\text {repair }}$ based on the minimization of a loss function (absolute percentage error, a.k.a. APE). This quantitative approach is coupled with visual comparison of model behavior to experimental data. The model whose inferred $k_{\text {repair }}$ value minimizes divergence between model and data behavior is thus chosen. A graphical representation of such a process is given below: 


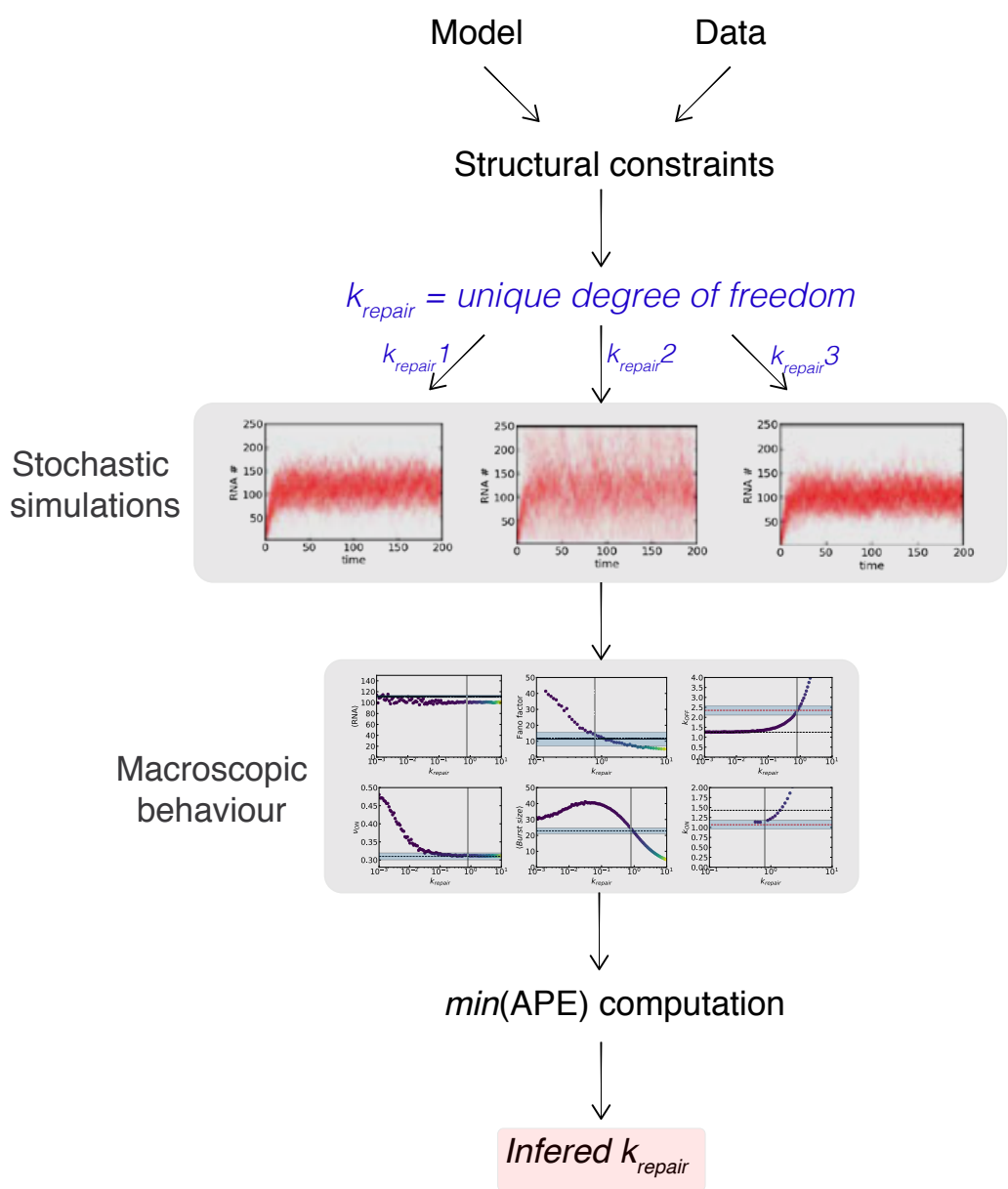

\subsubsection{Method of APE calculation}

To discriminate between models based on their macroscopic behavior, we need a measure that quantifies the discrepancy between model-derived results and experimental data. Because the macroscopic observations (Fano factor, $k_{O N}$, etc.) are of different orders of magnitude, we need a relative measure to avoid the largest parameters carrying the highest weight on the error. We chose to use the absolute percentage error (APE). The procedure is as follows:

Consider the vector $\mathbf{M} \equiv\left(\langle R N A\rangle, v_{O N}, B S, F F, k_{O N}, k_{O F F}\right)$. Thus, $\mathbf{M}_{\text {model }}$ and $\mathbf{M}_{\text {exp }}$ contain all the macroscopic observations from modeling and experiment respectively. $\mathbf{M}_{\text {model }}$ is a function of $k_{\text {repair }}$ or $k_{\text {incorpo }}$ equivalently. $\hat{k}_{\text {repair,APE }}$ is the best inferred value of the degree of freedom for a particular model. It is given by:

$$
\hat{k}_{\text {repair }, A P E}=\operatorname{argmin}_{k}\left|\frac{\mathbf{M}_{\text {exp }}-\mathbf{M}_{\text {model }}(k)}{\mathbf{M}_{\text {exp }}}\right|
$$


With $k \in\left[10^{-4}, 10\right]$, by assumption. This notation implies that we are minimizing the $\ell 1$ norm (sum of the vector components). In an operative manner, we simulated each model for 250 logarithmic distributed values of $k_{\text {repair }}$ using the previously described Gillespie algorithm.

To validate our approach, we devised an alternative loss function where the $\ell 1$ norm is computed using a non-biased (i.e symmetric) measure of relative prediction accuracy: the absolute $\log$ accuracy (ALA). The procedure is as follows:

$$
\hat{k}_{\text {repair }, A L A}=\operatorname{argmin}_{k}\left|\log \left(\frac{\mathbf{M}_{\text {model }}(k)}{\mathbf{M}_{\text {exp }}}\right)\right|
$$

With $k \in\left[10^{-4}, 10\right]$, by assumption.

\subsubsection{Results}

Using both the APE and ALA approaches, we obtained exactly the same results for parameter inference (except for model 1 for which $\hat{k}_{\text {incorpo,ALA }}=0.39$ ) and model selection. According to the APE-based approach for parameter inference and model selection, model 5 best recapitulates the macroscopic behavior observed in the data (Figure S21).

\subsection{Selection of Model 5}

Based on both the MLE- and APE-based approaches, model 5 best matches experimental FISH data and scRNA-seq data for Nanog. Model 5 is most similar to a transcription-coupled repair (TCR) mechanism in which repair events only occur while the gene is transcriptionally permissive. The key adjustment we make is the introduction of a higher transcription rate upon completion of repair. This modification is justified by our model selection process, as we find that the second-best model (model 4) also has an amplified transcription rate following Apex1 interaction and subsequent DNA repair. Interestingly, for the same value of $k_{\text {repair }}$, transcription-coupled repair (model 5) leads to a less significant increase in noise and better maintenance of mean as compared to model 4 in which repair can also take place in the OFF state (model 4). This implies that coupling of repair to transcription is the most efficient method of DNA repair in terms of minimizing excess transcriptional variability.

Several molecular mechanisms can lead to the amplified transcription rate that we have incorporated into model 5. Apex1 binding may both momentarily silence RNA transcription while also inducing increased chromatin supercoiling and chromatin remodelling. This could lead to a subsequent increase in transcription efficiency through increased initiation and/or RNApol II processivity. This feedback mechanism may be perceived as a homeostatic process, allowing maintenance of the mean mRNA production despite a perturbation in template integrity. It is important to note that the dynamic binding and unbinding of Apex1 triggers noise enhancement and mean 
maintenance more than the repair per-se. One implication of this is that other protein-DNA dynamic interactions may lead to unavoidable noise modulation through structural constraints like supercoiling. The strength of such modulation will depend on the kinetic rates of interaction. 


\section{Sensitivity analysis of TCR model (Model 5)}

\subsection{Modulation of $k_{\text {repair }}$ and $k_{\text {incorpo }}$ with fixed cooperativity}

We have seen that a model incorporating Apex 1 interaction with chromatin and an associated transcriptional amplification, can recapitulate an increase in noise without alteration of the mean number of mRNA produced by the Nanog gene expression system. We next asked how this behavior increase in noise without dramatic modification of mean expression - is related to the dynamics of Apex 1 binding and unbinding with chromatin. $k_{\text {repair }}$ and $k_{\text {incorpo }}$ are the kinetic rates describing such interaction.

We conducted a phase plane analysis of the system mean and Fano factor for both $k_{\text {repair }}$ and $k_{\text {incorpo }}$ (Fig S22A-B). We assume that the cooperativity is fixed and equal to the deduced cooperativity from the previous analysis using Nanog FISH data for $10 \mu M$ IdU. As expected, the Fano factor increases as $k_{\text {incorpo }}$ increases (for $k_{\text {incorpo }}$ lower than $\approx 1$ ). This suggests a positive dosedependent relationship between IdU and noise (Fig S22B).

We observe an inverse relation for $k_{\text {repair }}$, where noise increases as $k_{\text {repair }}$ decreases. Experimentally, we use a small-molecule inhibitor of the Apex 1 endonuclease domain (CRT0044876) to decrease $k_{\text {repair }}$. It is interesting to highlight that when $k_{\text {incorpo }}$ is higher than $\approx 1$ the Fano factor starts to decrease. These observations can be understood looking at equation (36) for the Fano factor. For $k_{\text {incorpo }}>1, v_{\text {on }}$ decreases slowly and the effective $\left\langle k_{R N A}\right\rangle$ starts to increase slowly as compared to when $k_{\text {incorpo }} \in[0.1,1]$ (Fig S22C-D). These changes are counteracted by a larger increase in $K_{O F F}$. The behavior for the mean number of RNA produced with increasing $k_{\text {incorpo }}>1$ can also be understood using the previous considerations: the decrease in mean corresponds to a decrease of the frequency in the ON state that is not counteracted by a strong enough cooperativity. All the results can be understood using the following formula for the Fano factor:

$$
F F=1+\frac{\left(1-v_{o n}\right) \cdot k_{R N A}}{K_{O N}+K_{O F F}+k_{d e c a y}}
$$

\subsection{Modulation of $k_{O N}$ and $k_{O F F}$ with fixed cooperativity}

We next wanted to define the parameter regime for $k_{O N}$ and $k_{O F F}$ in which homeostatic maintenance of mean expression is possible with transcription-coupled repair (model 5). For this analysis, simulations were run with values of $k_{O N}, k_{O F F} \in\left[10^{-3}, 10\right]$ for both the null model (standard 2state model, DMSO condition) and model 5. For the same values of $k_{O N}, k_{O F F}$, the fold change in mean of mRNA counts was calculated by comparing results of model 5 to the null model. This provides insight into how IdU treatment may impact expression of genes with different bursting kinetics. When $k_{O F F}>>k_{O N}$, the addition of IdU in Model 5 increases the average number of mRNA produced as compared to the null model (Fig S22E). This can be explained by a competition between the $O F F$ and $O N^{*}$ states and by the fact that in this portion of the phase space $k_{O N}<k_{O F F}<k_{\text {incorpo }}$ (and $k_{\text {incorpo }}<k_{\text {repair }}$ ). Therefore, the probability of presence in the $\mathrm{ON}_{2}$ 
state (transcriptionally more productive state), increases. This implies that IdU treatment would increase the mean of very lowly expressed genes. This was seen experimentally in bulk RNA-seq measurements of transcript abundance in mESCs as the $\approx 100$ genes that showed an increase in mean with IdU treatment were from the lowest expression regime (Fig S3). The exact inverse effect is observed in the upper left corner of the heatmap, where $k_{O N}>k_{O F F}>k_{\text {incorpo }}$. Thus for highly expressed genes, the effect of IdU on mean expression is minimal as seen experimentally.

\subsection{Cooperativity and noise enhancement}

When all the kinetics rates of the system are fixed, increasing the cooperativity, and thus the effective transcription rate, leads both to an increase in mean and Fano factor (Fig S22F).

\section{Testing of TCR model for other noise-enhanced genes}

In analyzing the Nanog gene expression we have found that IdU treatment leads to recruitment of DNA repair machinery while a gene is transcriptionally permissive (TCR model). This repair activity makes a second ON state accessible to the system. This state is characterized by an increased $k_{R N A}$ which is sufficient to recapitulate the experimental observations of mean maintenance with increased noise strength (Fano factor). We next asked whether the inferred TCR model can explain the experimental data collected for other noise-enhanced genes within the scRNA-seq dataset of mESCs treated with $10 \mu M$ IdU for 24 hours.

To simulate the TCR model for additional genes, estimates for the following parameters are needed: $k_{O N}, k_{O F F}, k_{R N A 1}$ (basal transcription rate in DMSO), $k_{\text {decay }}$, and $\left\langle k_{R N A}\right\rangle$ (effective transcription rate in IdU). To derive estimates of $k_{O N}, k_{O F F}, k_{R N A 1}$, and $\left\langle k_{R N A}\right\rangle$ we used a moments-matching technique described in $((83,84)$, where the first, second, and third exponential moments of the mRNA distributions in the DMSO and IdU conditions are used to calculate the parameters of a Poisson-beta distribution (describes 2-state model) that best fits experimental count data. The parameter estimates are derived in proportion to $k_{\text {decay }}$. Values of $k_{\text {decay }}$ were retrieved from an existing dataset of mRNA degradation rates in mESCs (92).

Of the 945 genes classified as highly variable with IdU treatment, 314 genes remained for downstream analysis based on availability of mRNA degradation rates and high confidence parameter estimates (Fig. S24A). With the above parameter estimates, we next computed the constraints on the cooperativity term and $k_{\text {incorpo }}$ as a function of $k_{\text {repair }}$ using the relationships derived in sections 2.5 and 2.6. There is again one remaining degree of freedom in our model system: $k_{\text {repair }}$. Using the MLE-based approach outlined in section 5.1, simulation results for a range of $k_{\text {repair }}$ values were compared against scRNA-seq data to identify $\hat{k}_{\text {repair,LLE }}$ for each of the 314 genes.

Once $\hat{k}_{\text {repair,LLE }}$ was identified, the macroscopic values of $K_{O N}$ and $K_{O F F}$ from simulation results were compared to experimentally derived estimates of $K_{O N}$ and $K_{O F F}$ from scRNA-seq data 
for each gene (Fig. S25). Overall, simulated values for macroscopic rates of promoter toggling in the IdU condition align with experimental results, indicating that the TCR model holds explanatory power for noise-enhanced genes beyond just Nanog. The use of a second, transcriptionallyenhanced ON state appears to be a unifying mechanism for maintenance of transcriptional homeostasis during DNA repair across a broad range of genes with different bursting kinetics. This suggests that the TCR mechanism for mean maintenance is robust to the initial bursting characteristics of a gene. 
bioRxiv preprint doi: https://doi.org/10.1101/2020.06.29.128439; this version posted June 30, 2020. The copyright holder for this preprint (which was not certified by peer review) is the author/funder, who has granted bioRxiv a license to display the preprint in perpetuity. It is made available under aCC-BY-NC-ND 4.0 International license.

\section{Supplementary Figures S1-S27}




\section{Supplemental Figure 1}

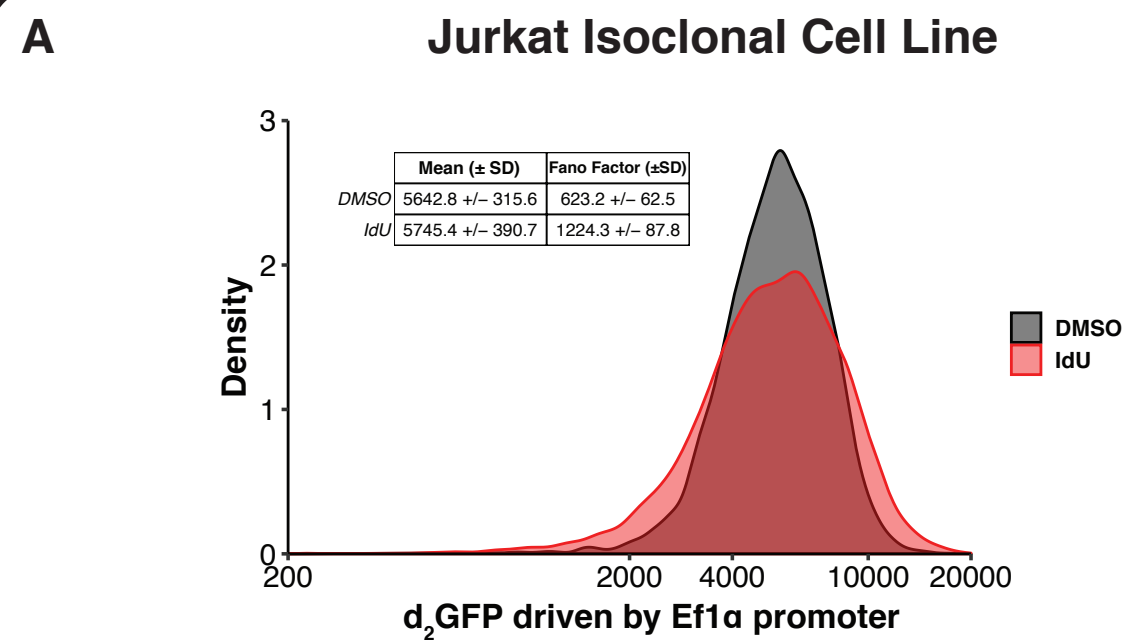

\section{B K562 Isoclonal Cell Lines}
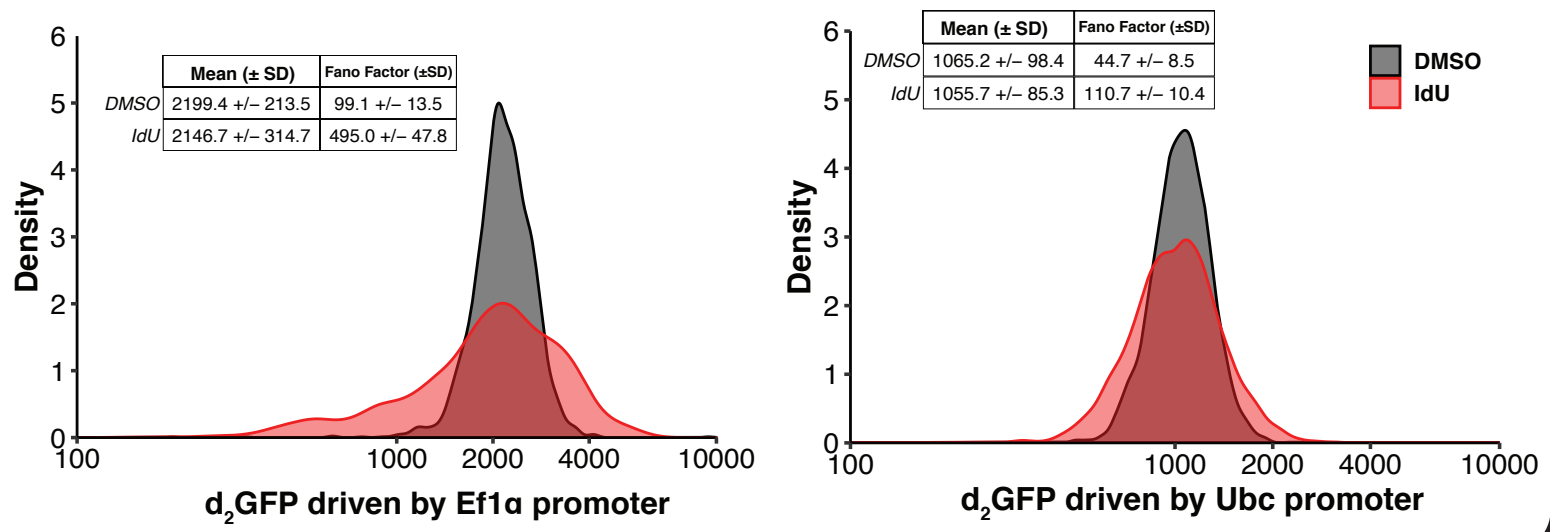

Figure S1: Nucleoside analog increases expression variability of housekeeping promoters in Jurkat and $\mathrm{K} 562$ cells.

(A) Representative flow cytomtery distributions of $\mathrm{d}_{2}$ GFP expression in an isoclonal population of Jurkat cells treated with either $20 \mu \mathrm{M}$ IdU or equivalent volume DMSO for 24 hours. Mean and SD are derived from 2 biological replicates. (B) Representative flow cytomtery distributions of $\mathrm{d}_{2} \mathrm{GFP}$ expression in isoclonal populations of K562 cells treated with either $20 \mu \mathrm{M}$ IdU or equivalent volume DMSO for 24 hours. Mean and SD are derived from 2 biological replicates. 


\section{Supplemental Figure 2}

A

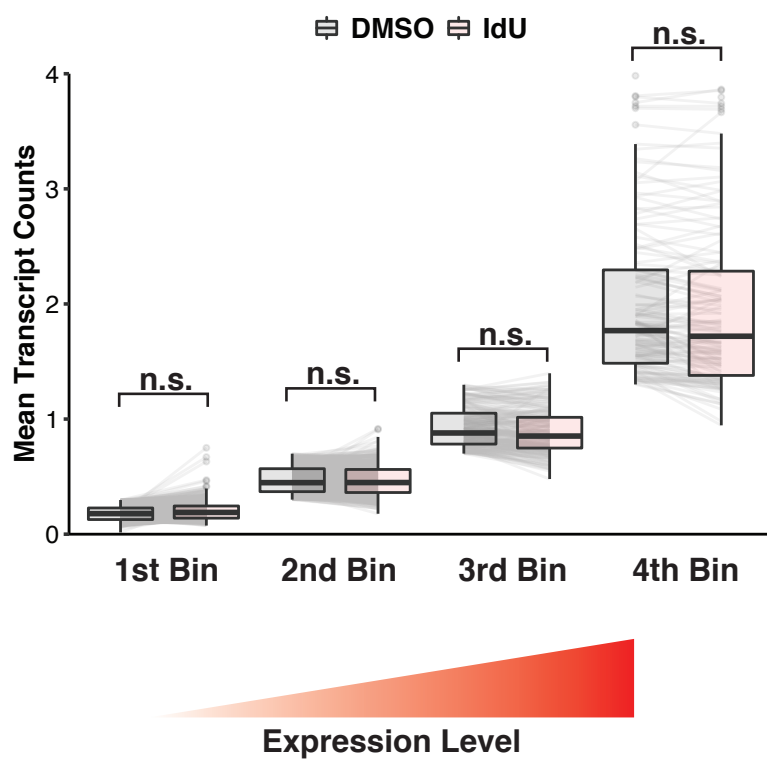

B

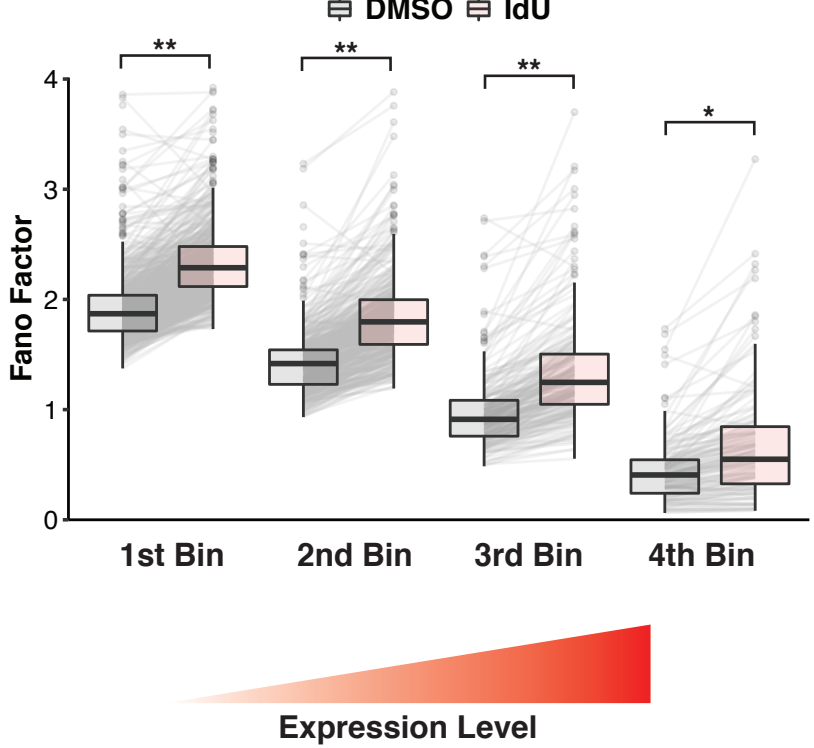


Figure S2: Noise enhancement occurs for genes across all expression levels.

4,578 genes from scRNA-seq dataset were binned into one of four groups (quartiles) based on mean expression level in DMSO condition. (A) Comparison of mean expression level for each gene in IdU and DMSO treatment groups. Boxplots show median \pm interquartile range of mean values for genes within each bin. Solid lines connect the same gene in the DMSO and IdU boxplots. P values were calculated using a two-tailed, paired Student's t test. (B) Comparison of Fano factor for each gene in IdU and DMSO treatment groups. Boxplots show median \pm interquartile range of Fano factors for genes within each bin. Solid lines connect the same gene in the DMSO and IdU boxplots. $\mathrm{P}$ values were calculated using a two-tailed, paired Student's $\mathrm{t}$ test. $* * \mathrm{p}<0.001, * \mathrm{p}=$ 0.0016 


\section{Supplemental Figure 3}

A

10uM IdU Treatment vs. DMSO

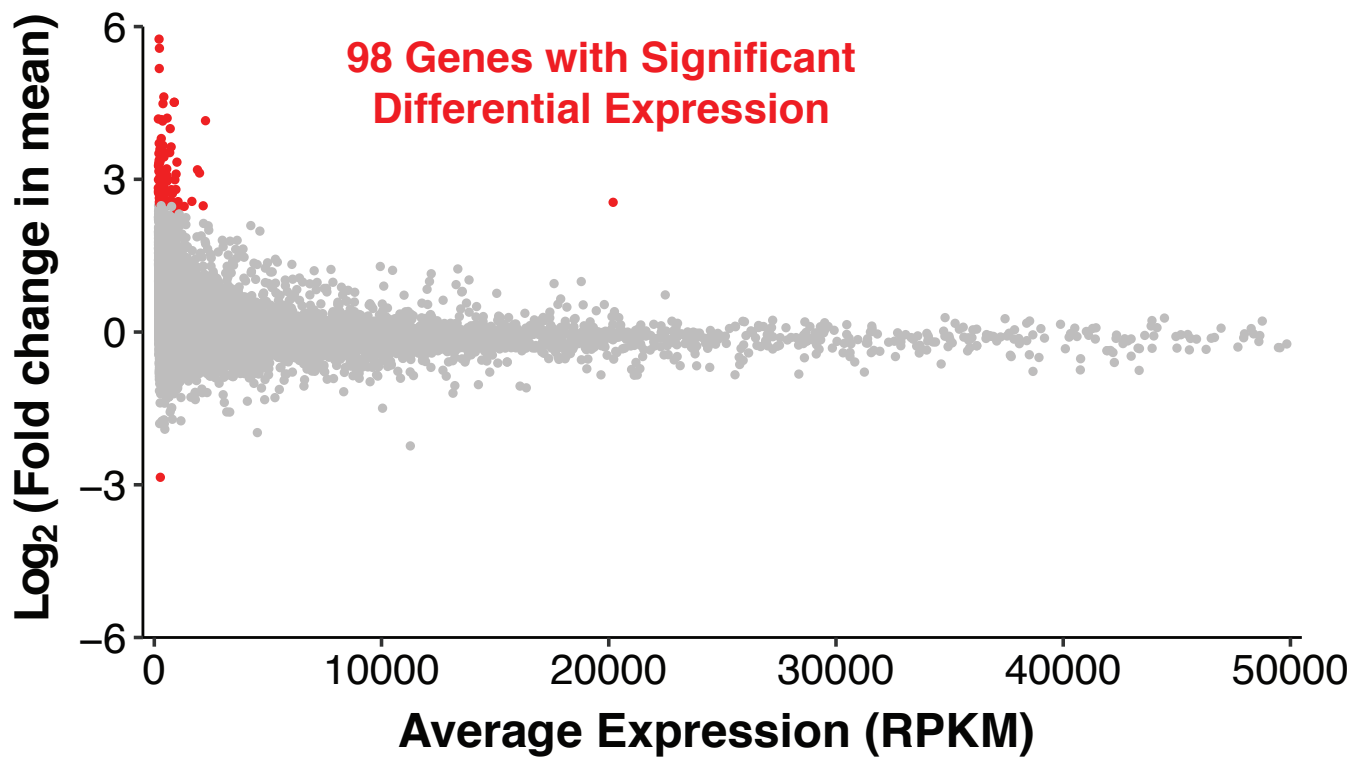

B

5uM IdU Treatment vs. DMSO

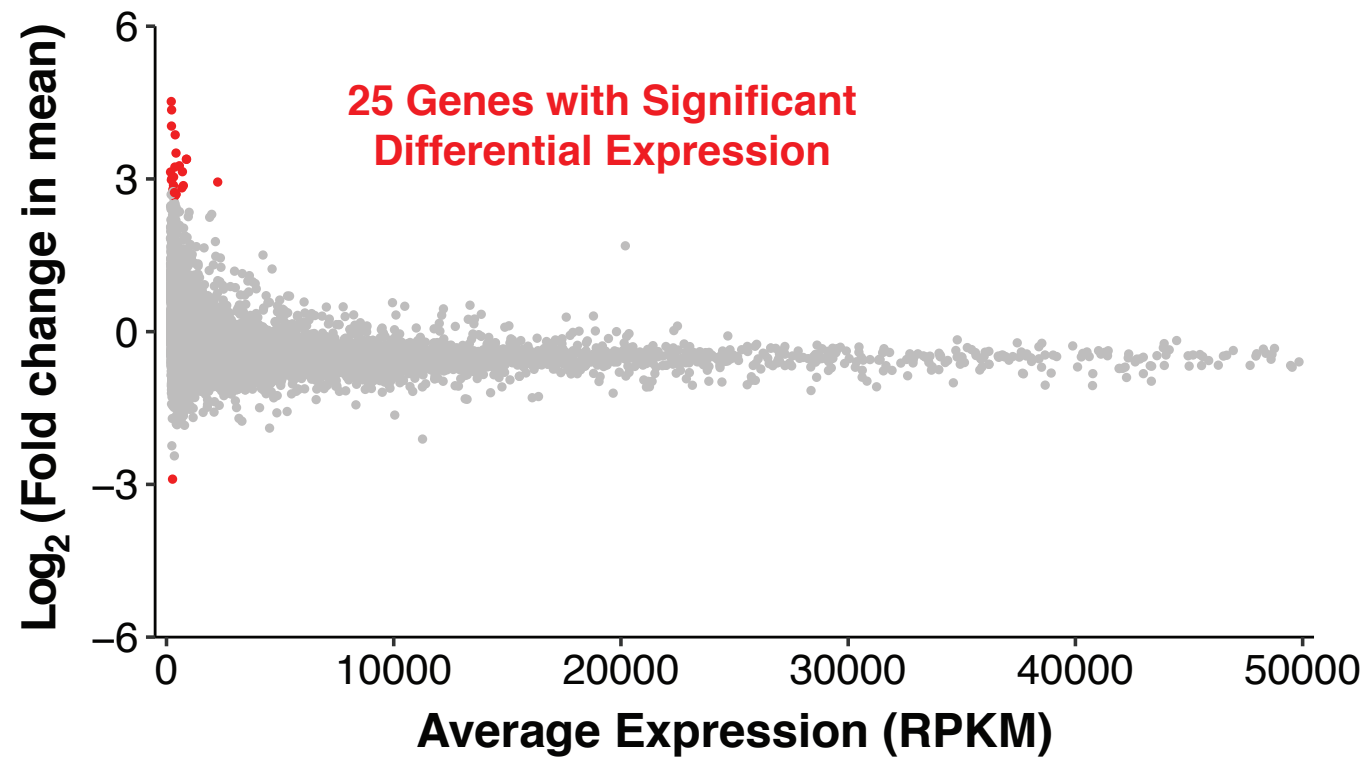


Figure S3: IdU causes minimal change in mean gene expression levels as measured by bulk RNA-seq.

Transcript abundances were normalized using ERCC spike-in counts. Differential mean testing was conducted with a threshold of fold change $>2$ and an FDR cutoff of 0.05 . Genes considered differentially expressed are highlighted in red. (A) Mean transcript abundance vs. fold change $\left(\log _{2}\right)$ in mean for 12,502 genes. Comparison is between $10 \mu \mathrm{M}$ IdU and DMSO treatments. 98 genes were identifed as differentially expressed. (B) Mean transcript abundance vs. fold change $\left(\log _{2}\right)$ in mean for 12,054 genes. Comparison is between $5 \mu \mathrm{M}$ IdU and DMSO treatments. 


\section{Supplemental Figure 4}

A

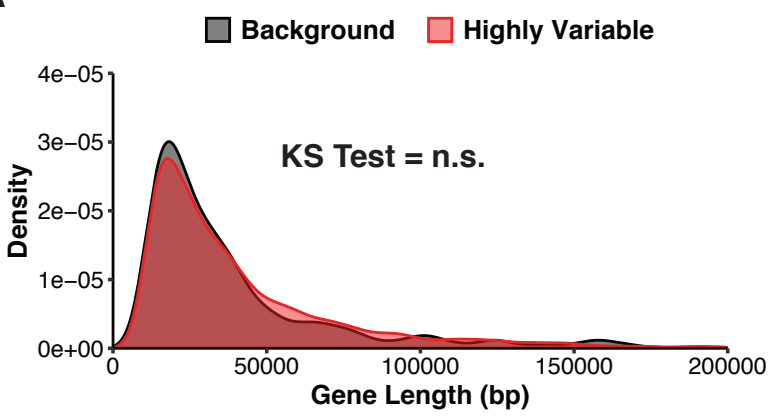

C

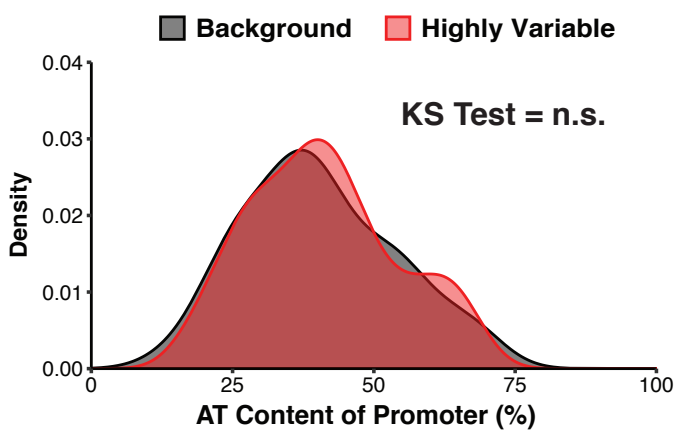

E

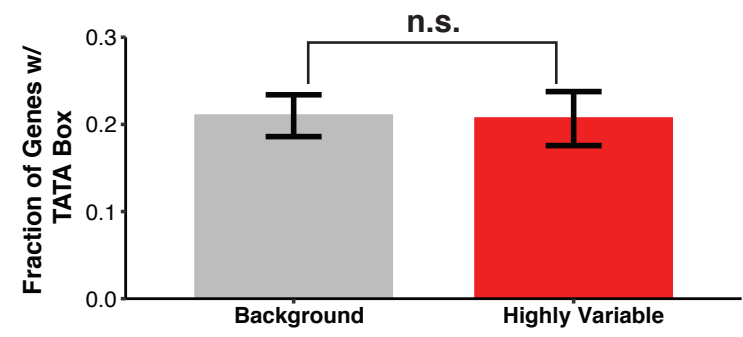

G

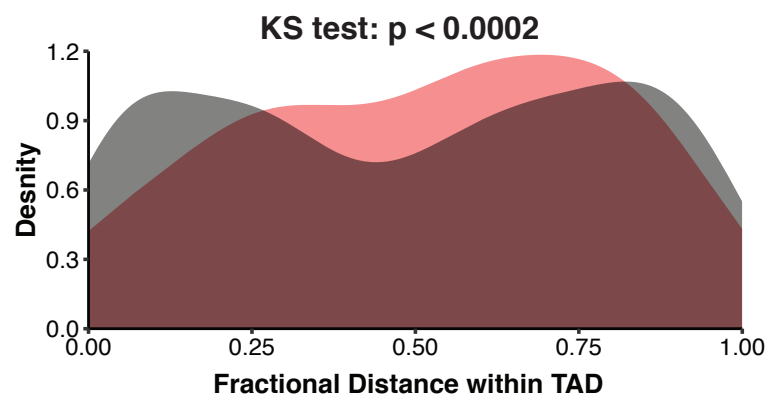

B

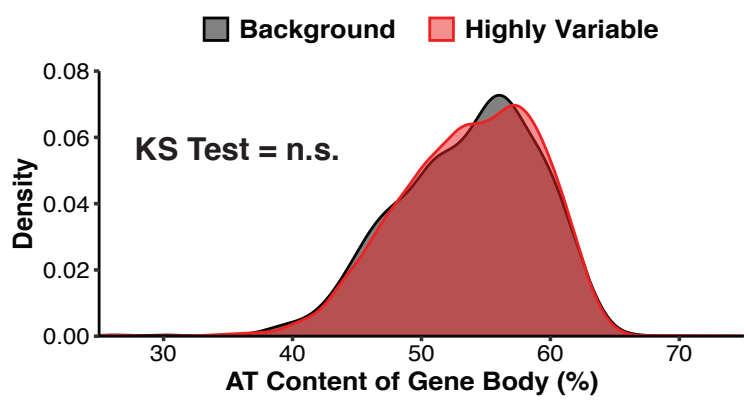

D

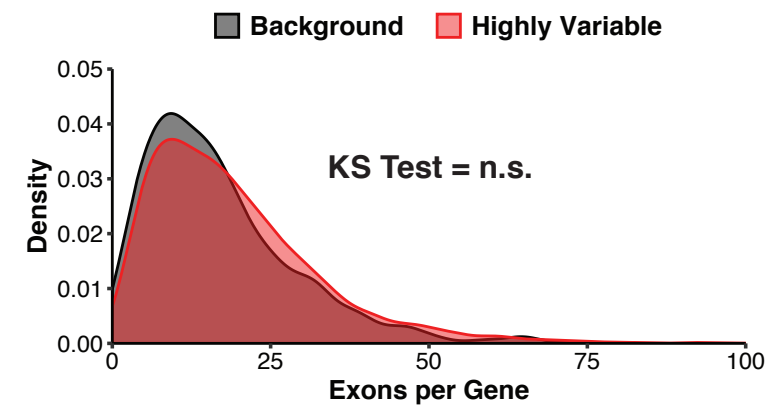

F

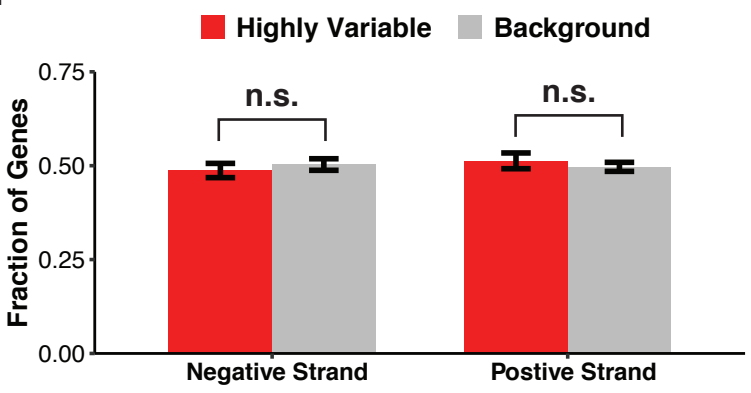


Figure S4: Noise-enhanced genes tend to be centrally located within topologically associating domains.

Comparisons are between 945 genes classified as highly variable and 3,513 genes classified as nonvariable (background) according to BASiCS algorithm. Gene characteristics and sequences were taken from Ensembl GRCm38 reference genome. (A) Distributions of gene lengths for highly variable and background genes. Length was calculated as distance between Ensembl gene start and end coordinates which correspond to outermost transcript start and end coordinates. (B) Percentage of base-pairs in gene body (based on gene start and end coordinates) that are A:T. (C) Percentage of base-pairs in 200bp region upstream of gene start that are A:T. (D) The number of exons was averaged over all transcripts associated with a gene. Distributions of average exon quantity for genes in the highly variable and background group were then plotted. (E) Fraction of genes with TATA sequence in $200 \mathrm{bp}$ region upstream of gene start. Data represent mean and SD from bootstrapping procedure with 10,000 resamplings of 100 genes from each group with replacement. (F) Fraction of genes whose coding sequence is located on negative and positive strands. Data represent mean and SD from bootstrapping procedure with 10,000 resamplings of 100 genes from each group with replacement. (G) Fractional distance of gene within TAD was calculated as (gene start coordinate - TAD start coordinate)/(TAD end coordinate - TAD start coordinate). Coordinates of TAD boundaries in mESCs were taken from Elphege et al. (89). 


\section{Supplemental Figure 5}

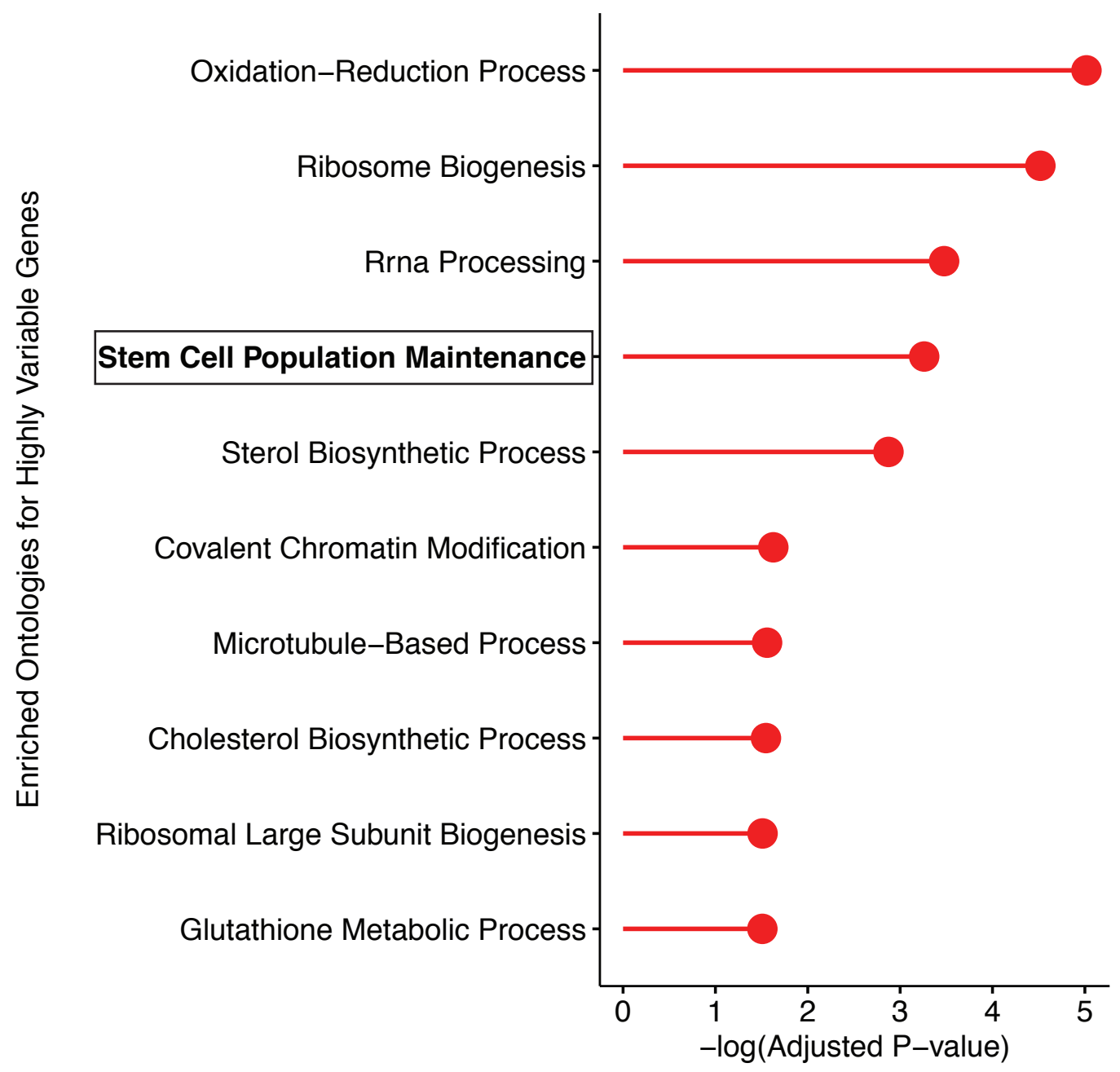

Figure S5: Ontology analysis of variably expressed genes shows enrichment for housekeeping and pluripotency maintenance pathways.

DAVID v6.8 was used to identify enriched ontologies among the 945 genes classified as highly variable according to BASiCS algorithm. 


\section{Supplemental Figure 6}

A

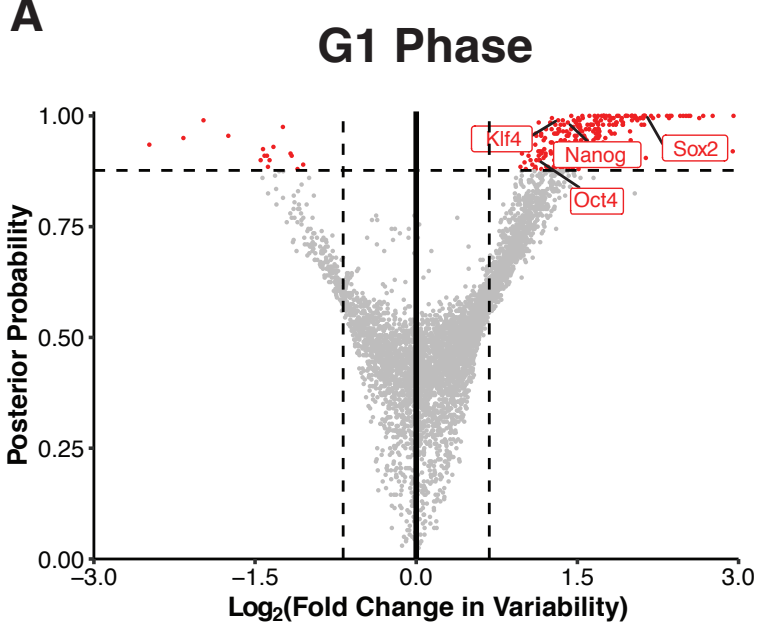

C

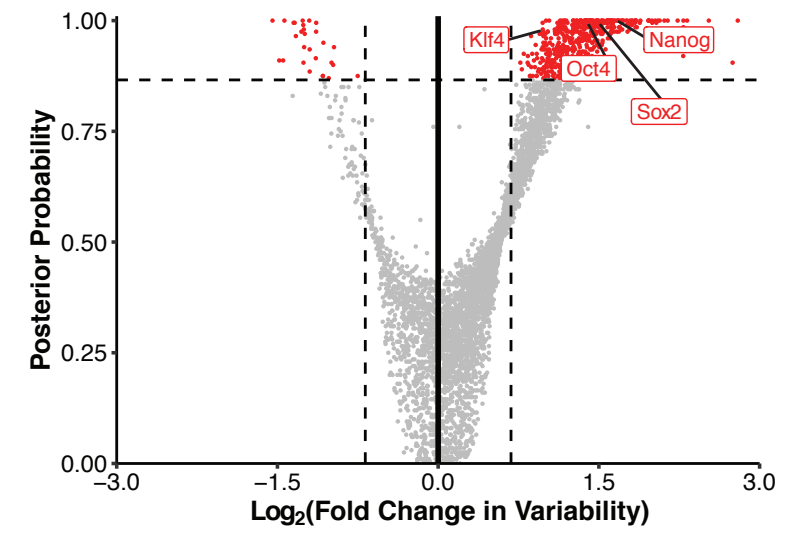

B

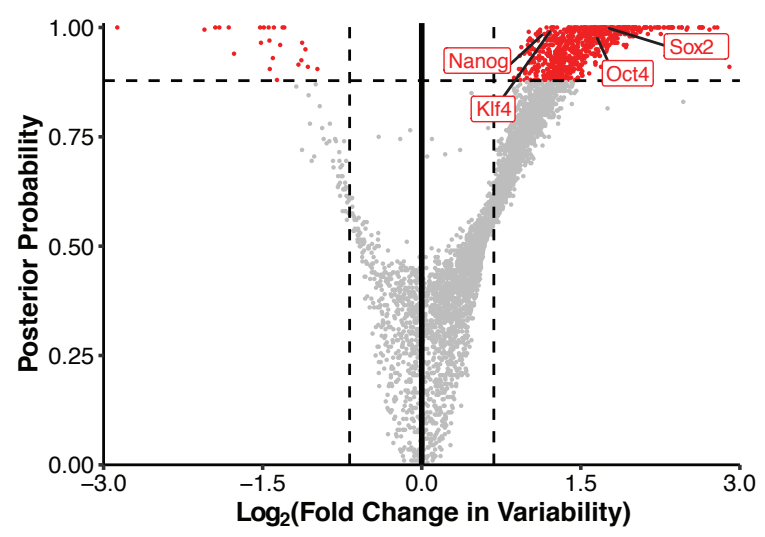

Figure S6: Noise-enhancement of pluripotency factors occurs in all three phases of the cell cycle.

A total of 1556 cells in the scRNA-seq dataset were classified into one of three cell-cycle phases. Differential variability testing was then conducted between cells in the DMSO and IdU treatment groups with the same cycle classification. 


\section{Supplemental Figure 7}

DMSO

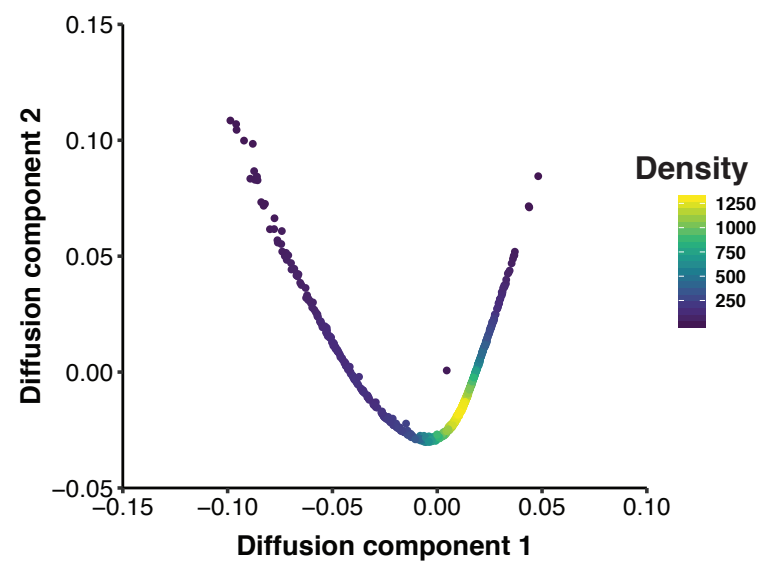

IdU

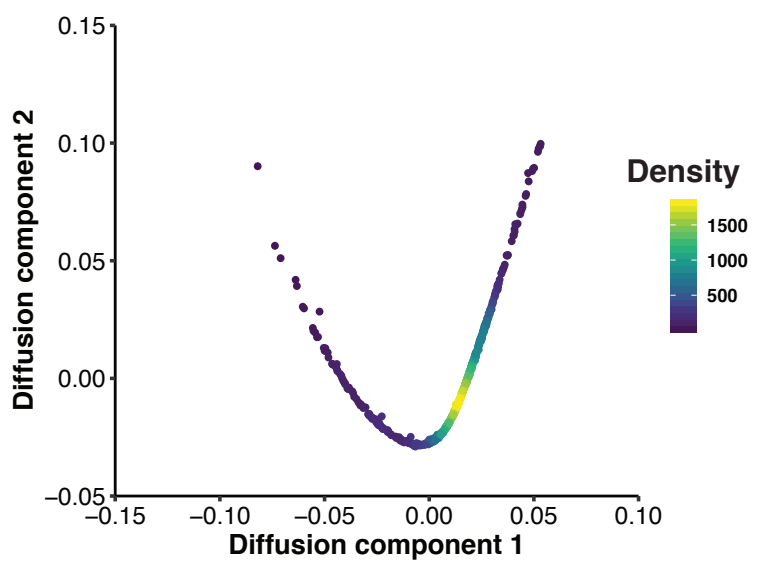

Figure S7: Transcript variability is not caused by bifurcation of mESCs into separate developmental lineages.

Pseudotime analysis of IdU-treated cells shows no differentiation of mESCs into separate developmental lineages. 


\section{Supplemental Figure 8}

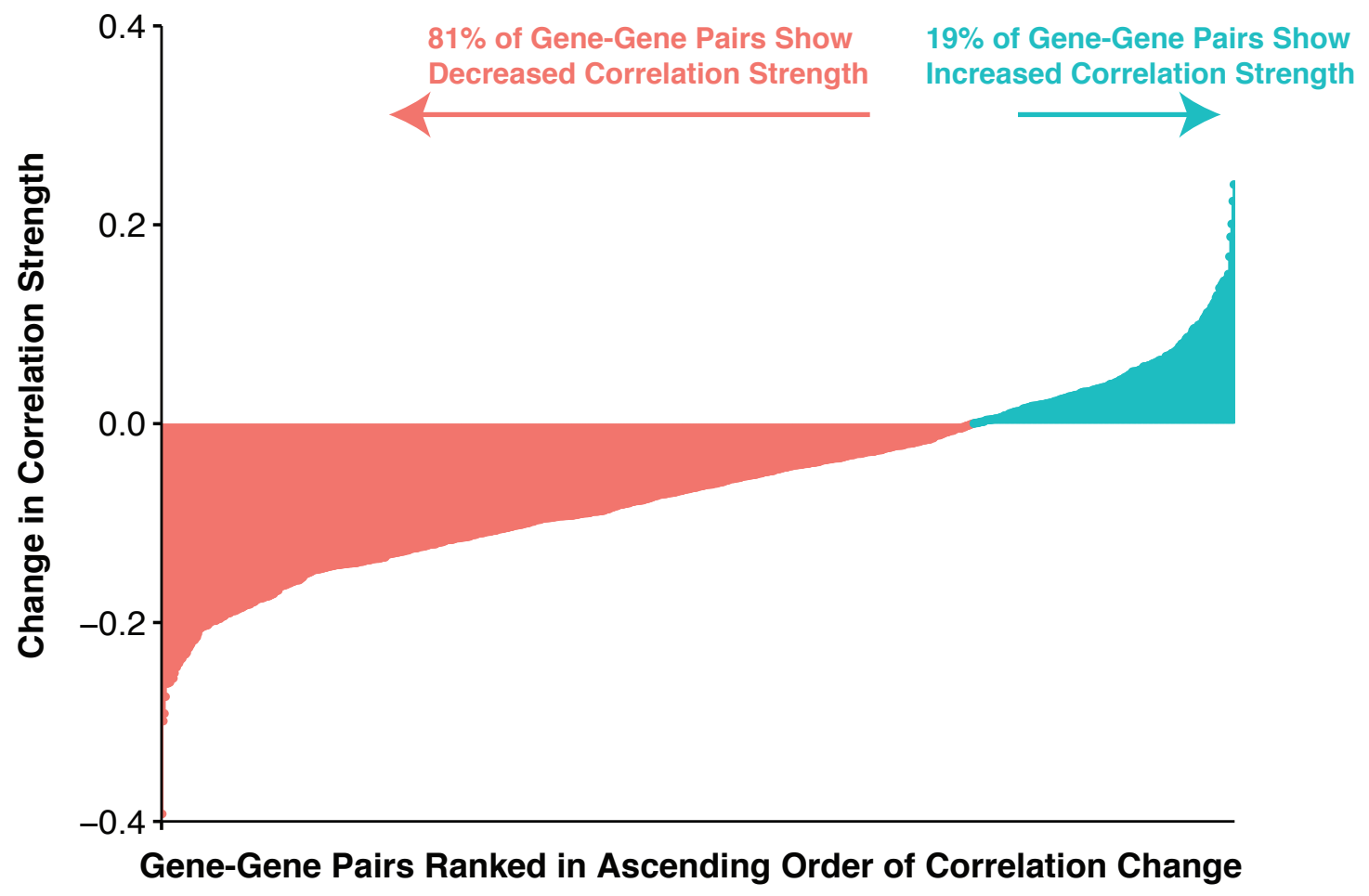

Figure S8: Majority of gene-gene pairs show a decrease in correlation strength.

The Pearson correlation of expression for 923,521 (961 x 961) gene-gene pairs were compared between DMSO and IdU treatment groups. For each gene-gene pair, the absolute value of the correlation strength in DMSO was subtracted from the absolute value of the correlation strength in IdU. Negative values indicate loss of correlation in expression. 


\section{Supplemental Figure 9}

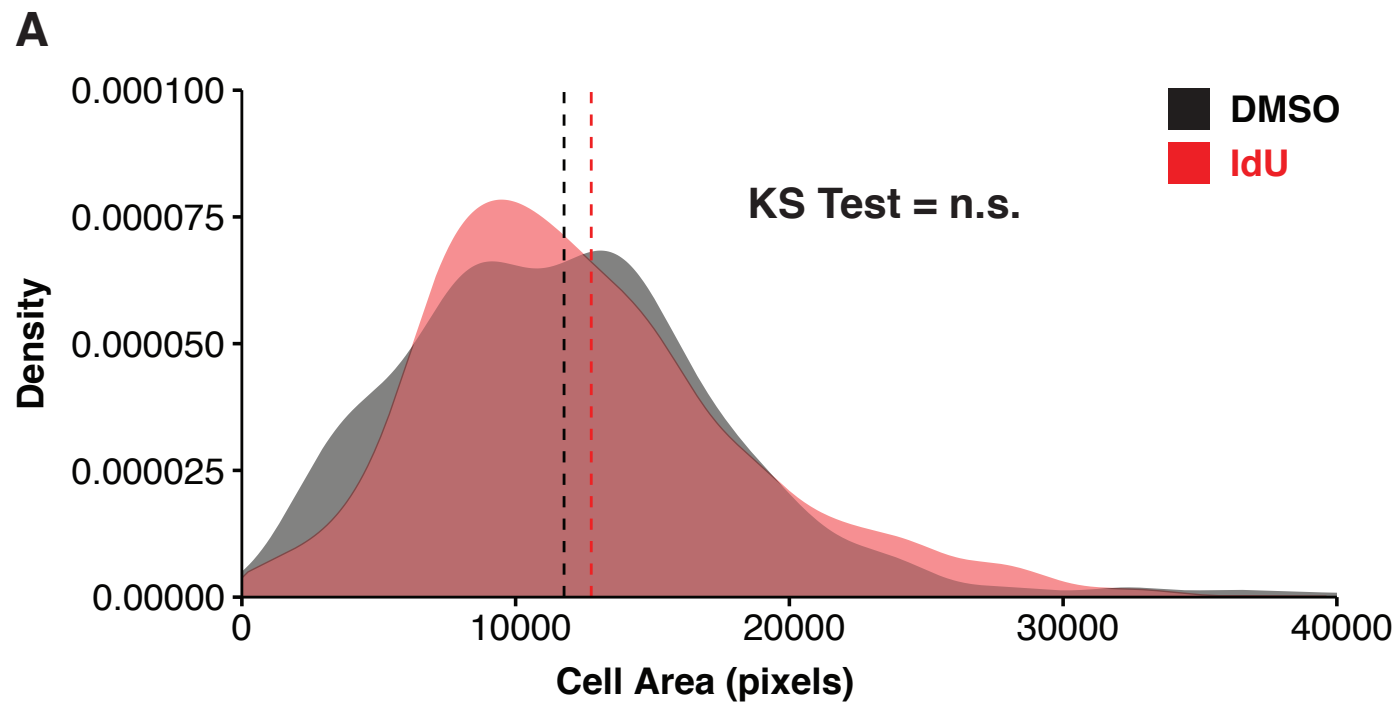

B
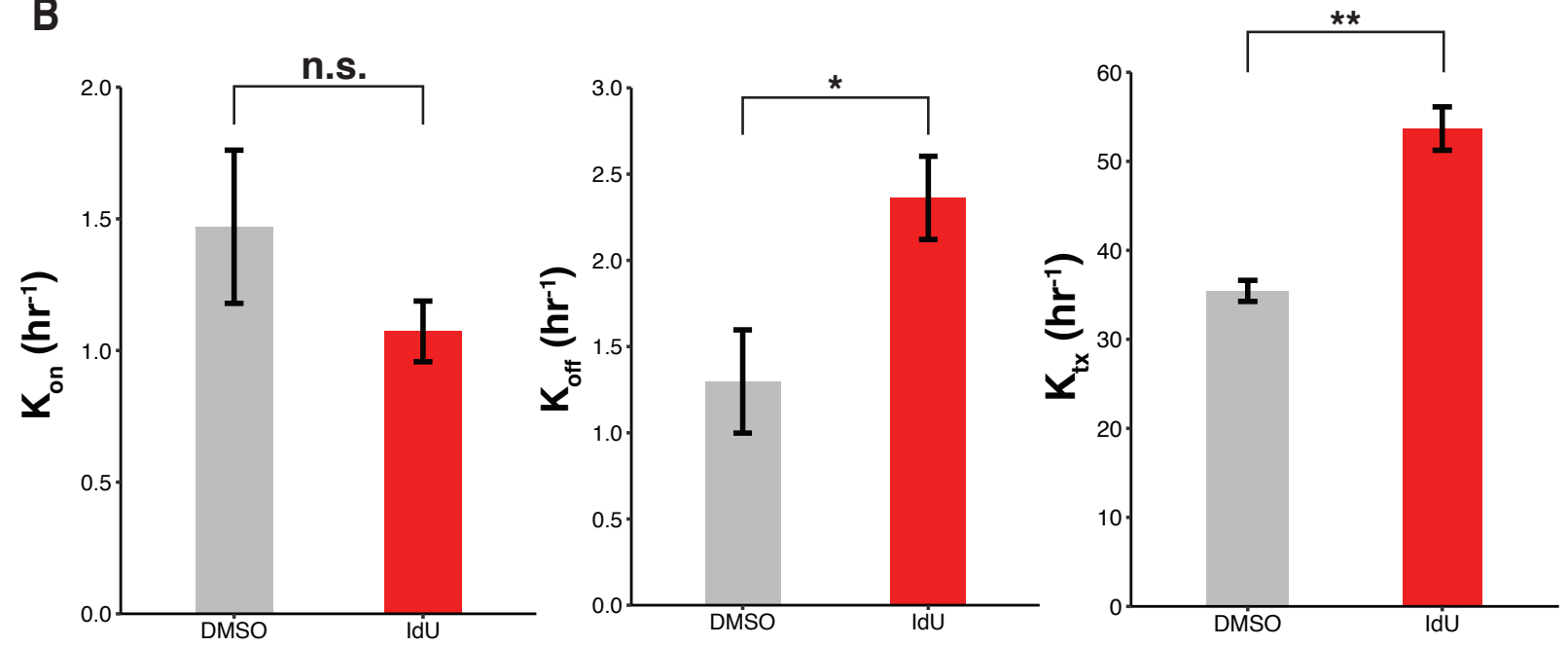

Figure S9: Shortened burst duration and increased transcription rate causes enhanced cellto-cell variability in Nanog mRNA counts.

(A) Distribution of cell sizes for analyzed cells in DMSO and IdU conditions. Cell size was calculated as number of pixels within segmented cell boundary. Dashed lines represent means of each distribution. Data represent pooling of cells from all four biological replicates for each condition. KS test shows no significant difference between cell size distributions. (B) Inference of parameters for 2-state model of transcription. $\mathrm{P}$ values were calculated using a two-tailed, unpaired Student's t test. ${ }^{*} \mathrm{p}=0.0017,{ }^{* *} \mathrm{p}=0.0001$ 
bioRxiv preprint doi: https://doi.org/10.1101/2020.06.29.128439; this version posted June 30, 2020. The copyright holder for this preprint (which was not certified by peer review) is the author/funder, who has granted bioRxiv a license to display the preprint in perpetuity. It is made available under aCC-BY-NC-ND 4.0 International license.

\section{Supplemental Figure 10}
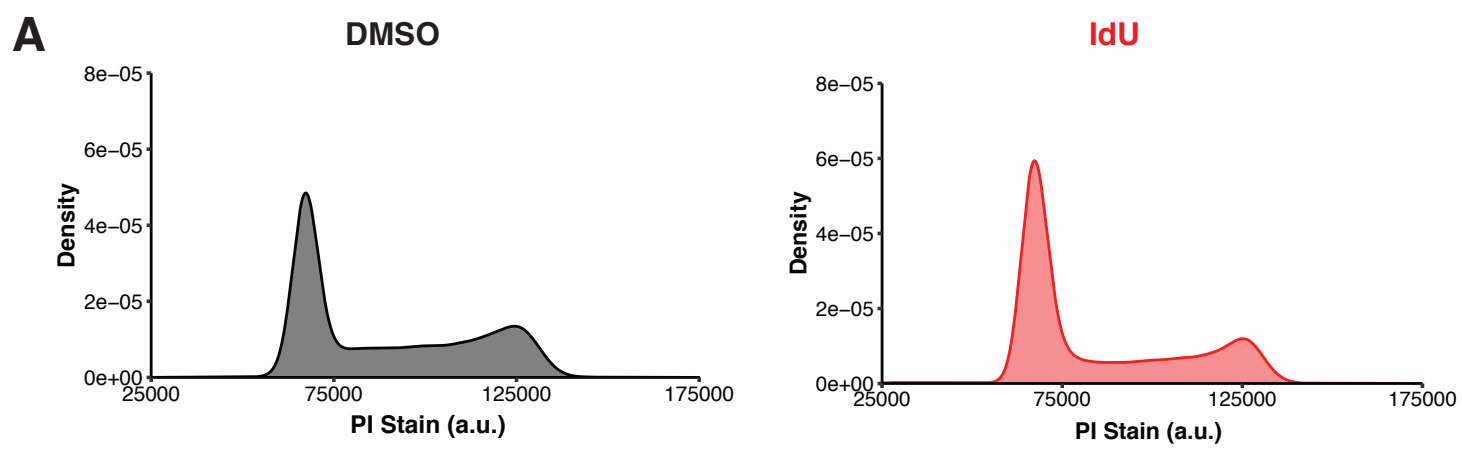

B

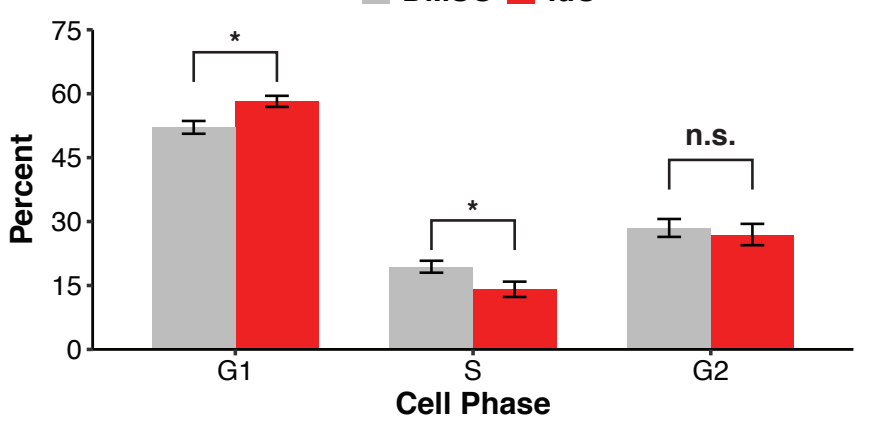

C

G1 Phase

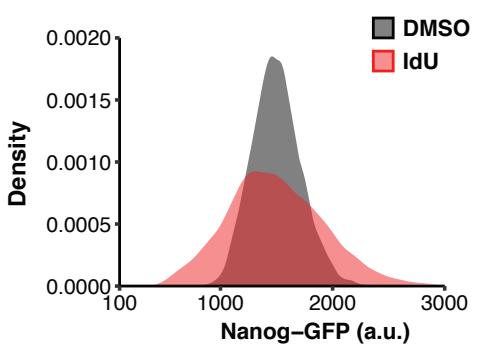

S Phase

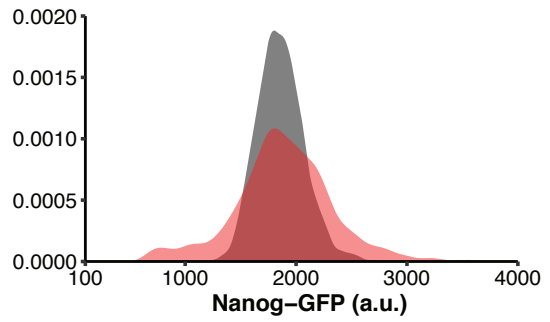

G2 Phase

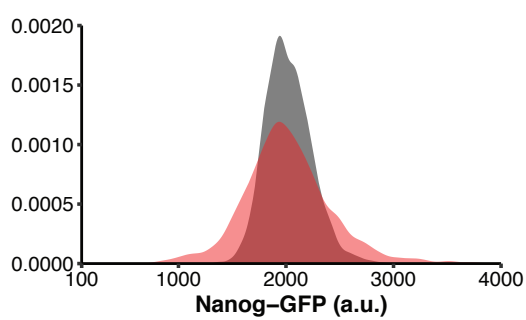

D

n.s.

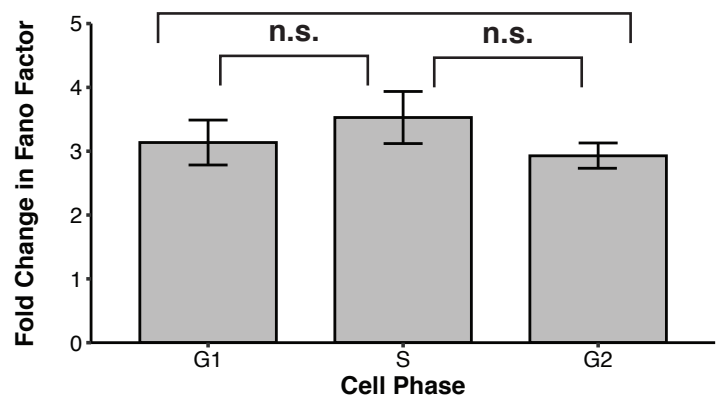


Figure S10: Noise-enhancement of Nanog protein expression is independent of cell-cycle state.

(A) Representative flow cytometry distributions of propidium iodide staining for Nanog-GFP mESCs treated with either DMSO or $10 \mu \mathrm{M}$ IdU for 24 hours. No signs of aneuploidy are visible, indicating transcriptional variability is not due to cell-to-cell variability in gene copy numbers. (B) Percent of cells in each phase of the cell cycle for DMSO and IdU treatments based on propidium iodide staining. IdU treatment slightly slows entry into S phase. Data represent mean and SD of three biological replicates. P values were calculated using a two-tailed, unpaired Student's t test. $* p<0.01$ (C) Representative flow cytometry distributions of Nanog-GFP for mESCs within the G1, S and G2 phases of the cell cycle. mESCs were treated with $10 \mu \mathrm{M}$ IdU or equivalent volume DMSO for $24 \mathrm{~h}$ followed by propidium iodide staining. (D) IdU-induced noise-enhancement of Nanog-GFP protein levels is unchanged across all three phases of the cell cycle. Nanog-GFP Fano factor with IdU treatment was normalized to DMSO control for calculation of fold change. Data represent mean and SD of three biological replicates. P values were calculated using a two-tailed, unpaired Student's t test. 


\section{Supplemental Figure 11}

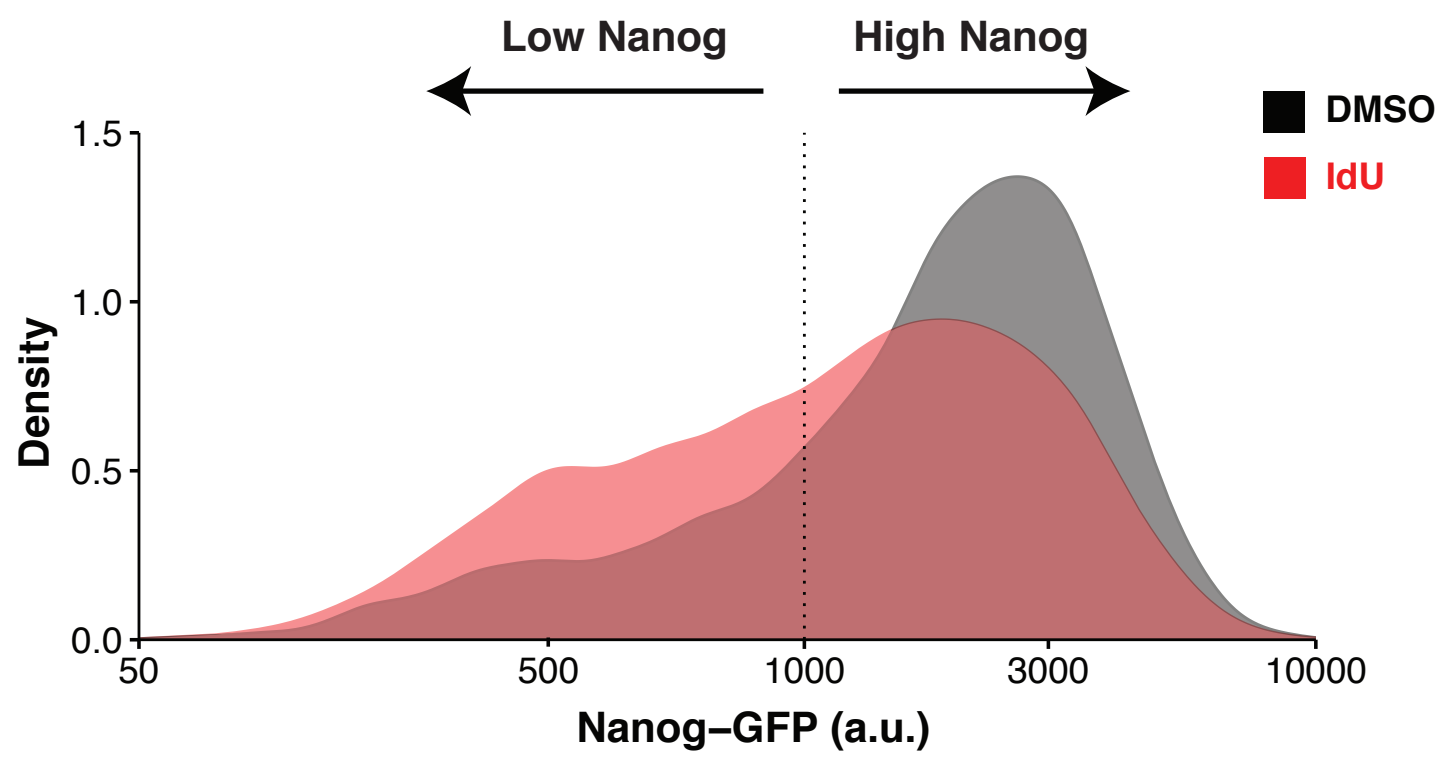

Figure S11: Increased transcriptional noise drives a greater number of mESCs into the lowNanog state while cultured in serum/LIF.

Flow cytometry distribution of Nanog-GFP expression for mESCs cultured in serum/LIF and treated with $10 \mu \mathrm{M}$ IdU or equivalent volume DMSO for $24 \mathrm{~h}$. Data is pooled from three biological replicates. 


\section{Supplemental Figure 12}
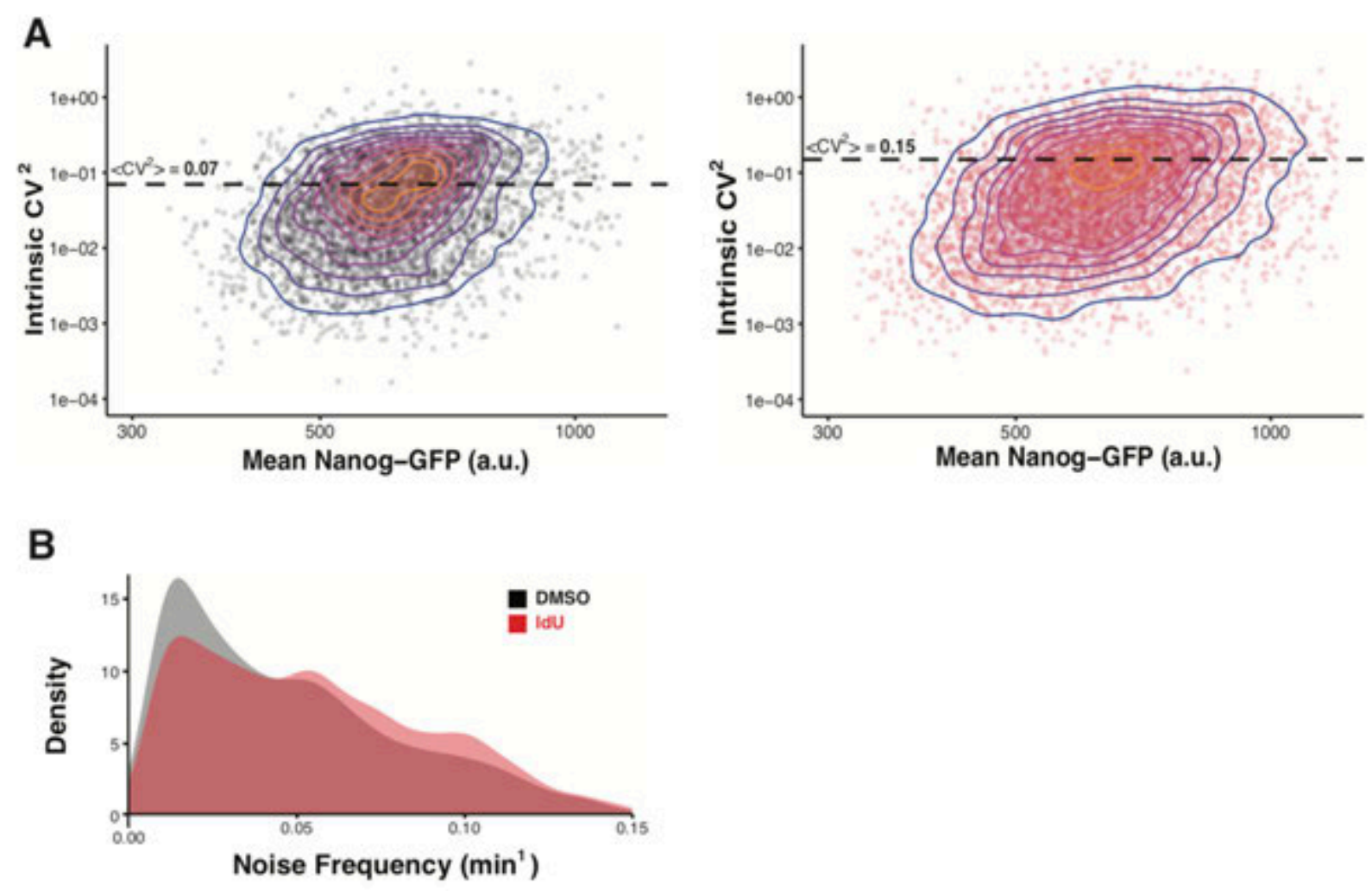

Figure S12: Time-lapse imaging demonstrates that altered kinetics of promoter toggling cause individual cells to experience larger fluctuations in Nanog protein expression.

(A) Each point represents a single-cell fluorescence trajectory (DMSO on left, $\mathrm{n}=1513$; IdU on right, $\mathrm{n}=1414$ ). Single-cell fluorescence trajectories were detrended by subtracting timedependent population average for Nanog-GFP fluorescence. The mean Nanog-GFP fluorescence for each raw trajectory is then plotted versus the $\mathrm{CV}^{2}$ of the detrended version of the trajectory to isolate intrinsic noise. The dashed lines represent the average intrinsic $\mathrm{CV}^{2}$ of all trajectories for each treatment group. Time-lapse imaging shows that for individual cells the magnitude of Nanog protein fluctuations increases with IdU treatment. (B) Distributions of noise frequencies from autocorrelation functions of each detrended trajectory. Noise frequency is calculated as the inverse of the autocorrelation time $\left(\tau_{1 / 2}\right)$. Shorter but more productive transcriptional bursts with IdU treatment pushes the frequency content of Nanog-GFP fluctuations to higher spectra. 


\section{Supplemental Figure 13}

Time

Low Nanog Bin

$0 \mathrm{hrs}$

$7 \mathrm{hrs}$

14 hrs

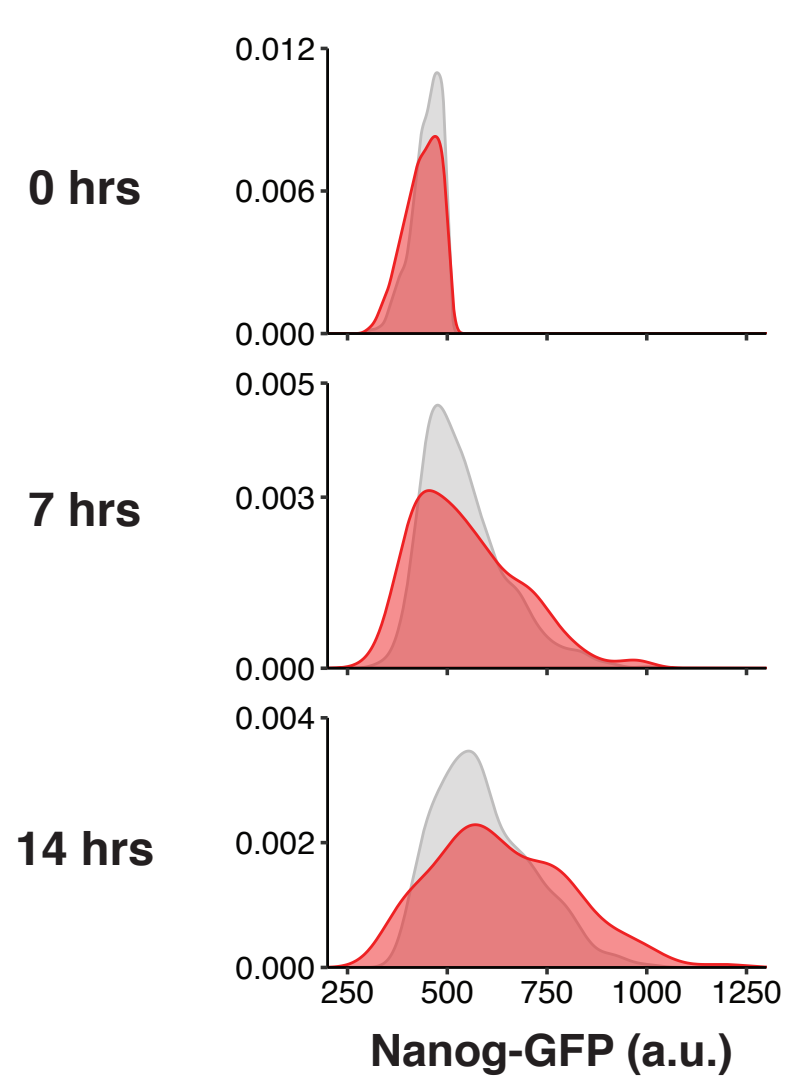

High Nanog Bin
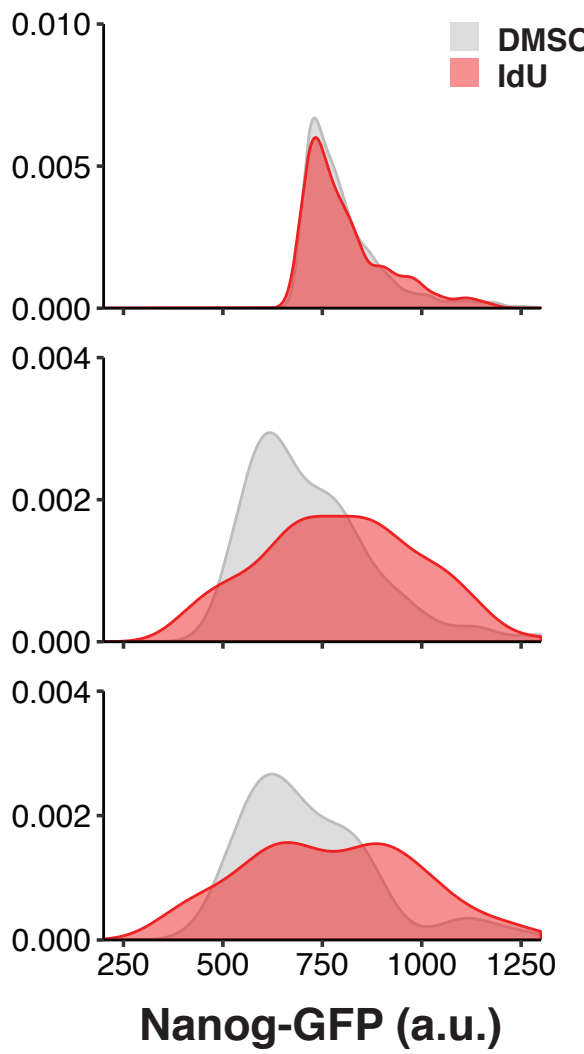

Figure S13: Amplification of expression fluctuations occurs independently of starting Nanog level.

Single-cell trajectories whose starting fluorescence value was below 500 a.u. or above 700 a.u. were binned into low and high groups respectively. Only trajectories whose starting point coincided with addition of DMSO or IdU at time zero were used. Distributions of trajectory fluorescence values at zero, seven, and 14 hours into treatment conditions are shown. By 14 hours into IdU treatment, there is visible interconversion of cells between the low and high Nanog states, indicating that memory of initial Nanog expression level is erased. This precludes the possibility that noise enhancement is due to promoter mutations that create sub-populations of cells with stable expression of Nanog at low and high levels. 


\section{Supplemental Figure 14}

A

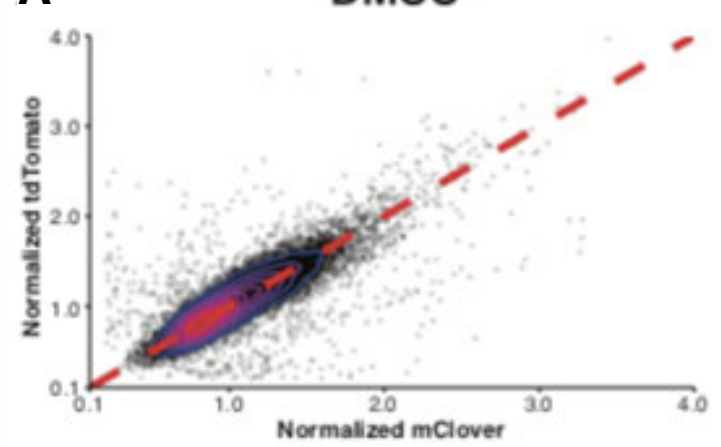

IdU

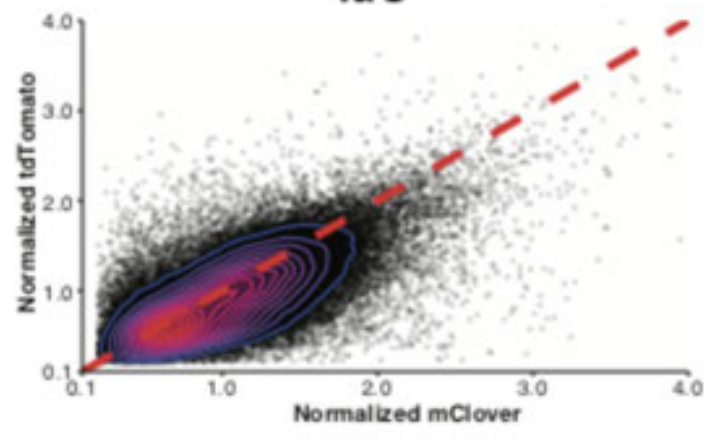

B

- DMSO - IdU

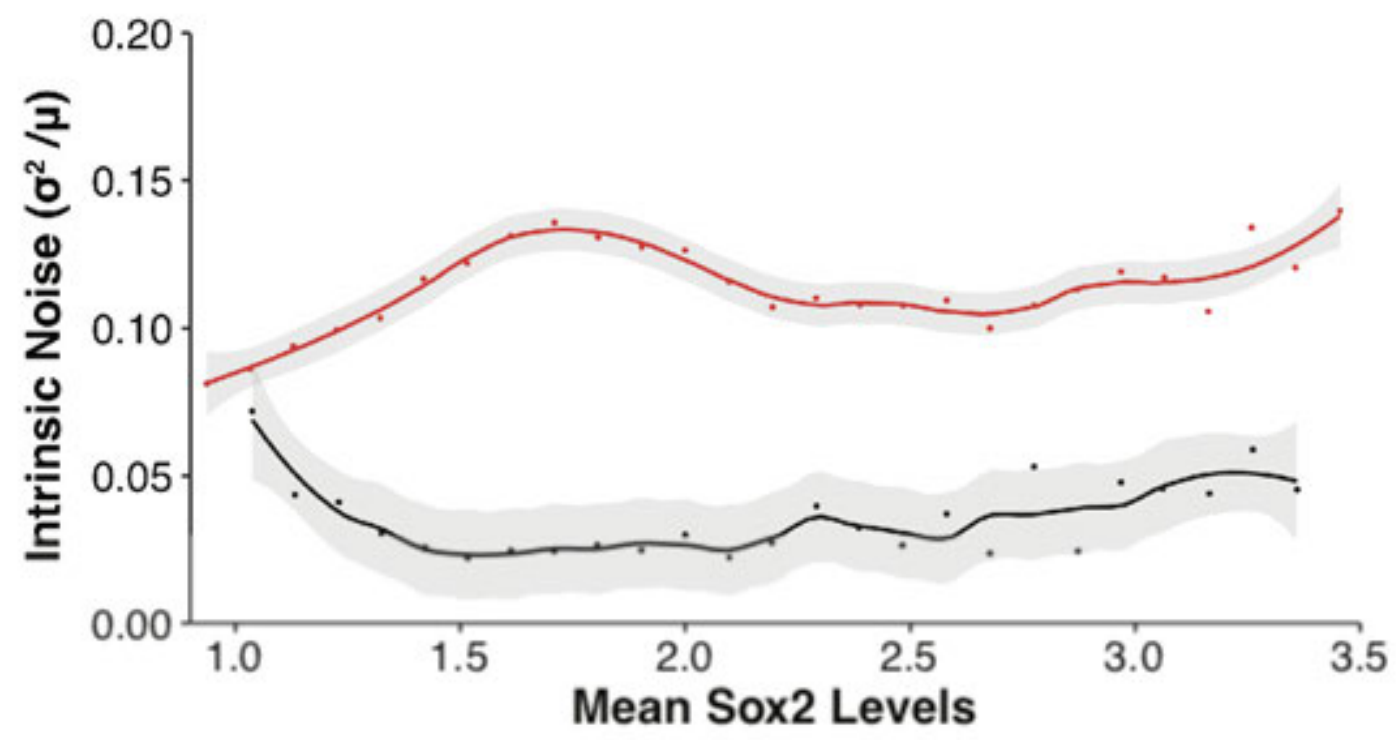

Figure S14: IdU treatment increases intrinsic noise of Sox2 expression.

(A) Flow cytometry dot-plot of mESCs with Sox 2 dual color tags. Dashed red line has slope of one. mCLover and tdTomato fluorescence values were normalized to population average. Data shown is pooled from three biological replicates. (B) Cells were binned according to total Sox 2 expression from both alleles. Each point represents the intrinsic noise (Fano factor) of Sox 2 expression for cells within a particular bin. Grey shadings represent $95 \%$ confidence intervals as determined by bootstrapping. Smooth lines are produced from loess regression. IdU increases Sox2 intrinsic noise across all expression levels. 
bioRxiv preprint doi: https://doi.org/10.1101/2020.06.29.128439; this version posted June 30, 2020. The copyright holder for this preprint (which was not certified by peer review) is the author/funder, who has granted bioRxiv a license to display the preprint in perpetuity. It is made available under aCC-BY-NC-ND 4.0 International license.

\section{Supplemental Figure 15}
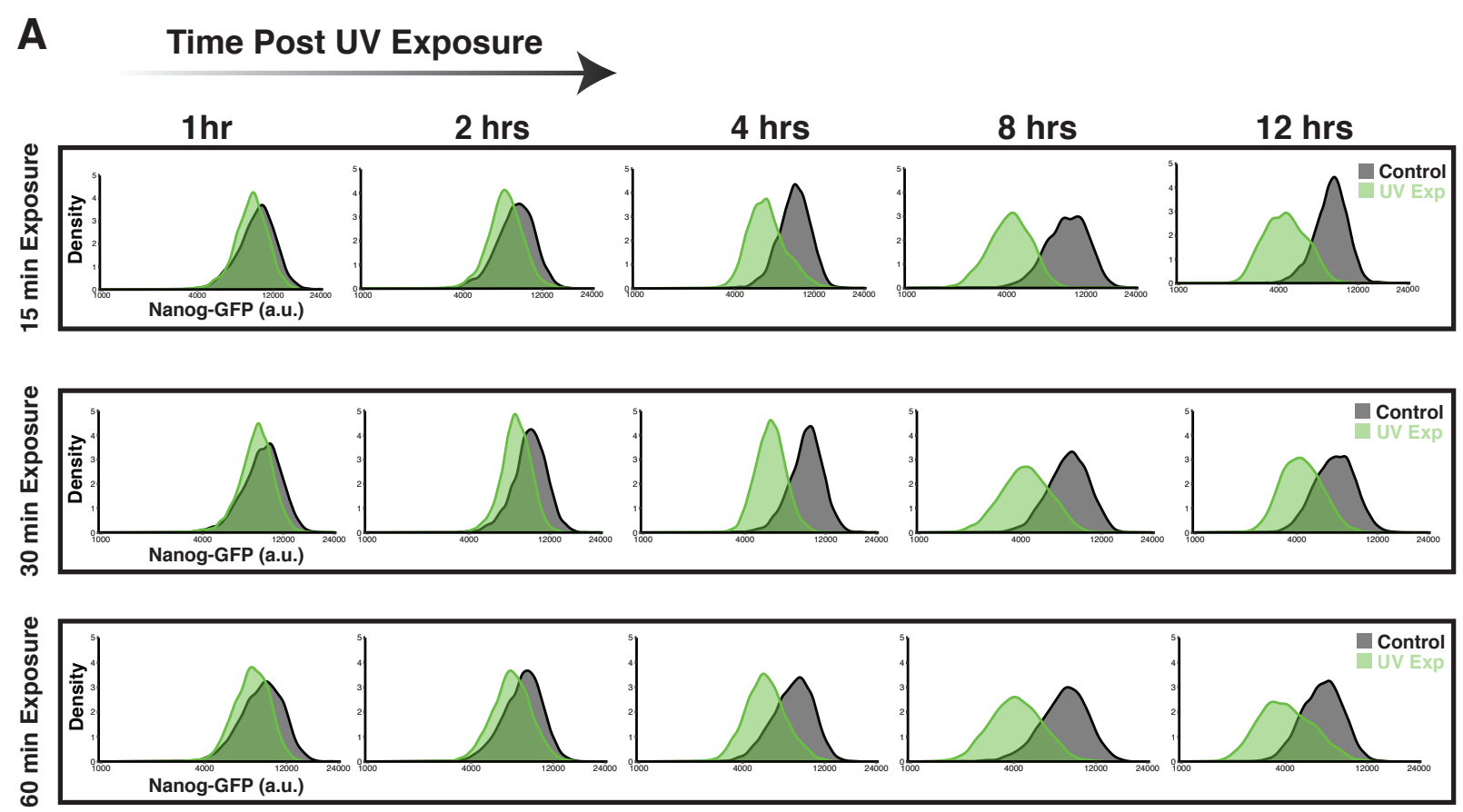

B

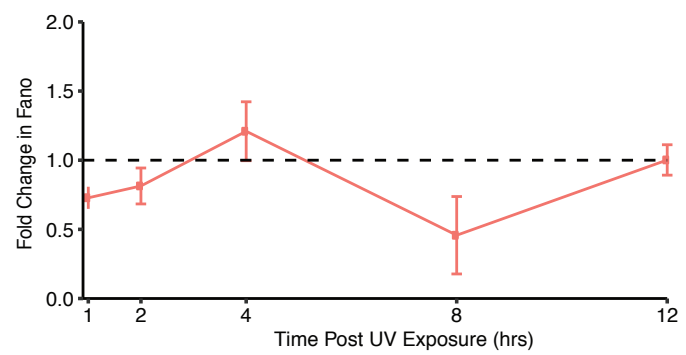

60 min UV Exposure

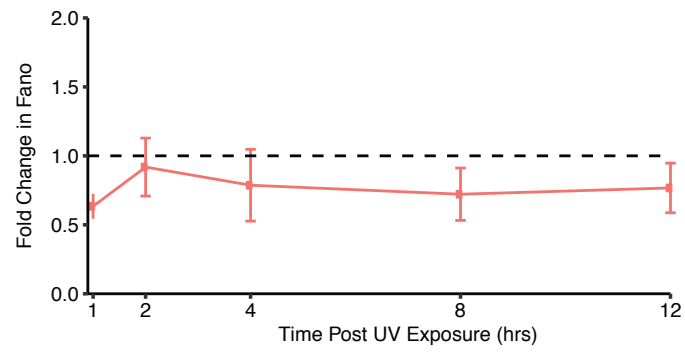

30 min UV Exposure

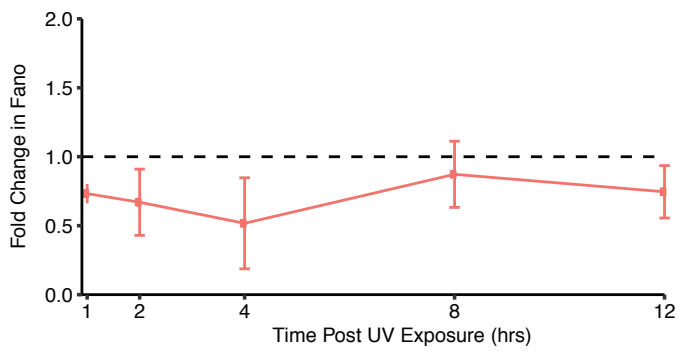




\section{Figure S15: UV-stress reduces Nanog mean and Fano factor.}

(A) Representative flow cytometry distributions of Nanog-GFP expression from UV-exposed (green) and control (grey) cell populations. Cells were analyzed one, two, four, eight, and 12 hours post exposure. (B) For each exposure group (15, 30, and 60 minutes), the fold change in Fano factor is calculated as the Fano factor for Nanog in the UV-exposed population normalized to the Fano factor of its respective control population. Data points represent mean and SD of two biological replicates. Across all time points (except 4 hour point in 15 minute exposure group) UV stress reduces the Fano factor of Nanog. 


\section{Supplemental Figure 16}

A

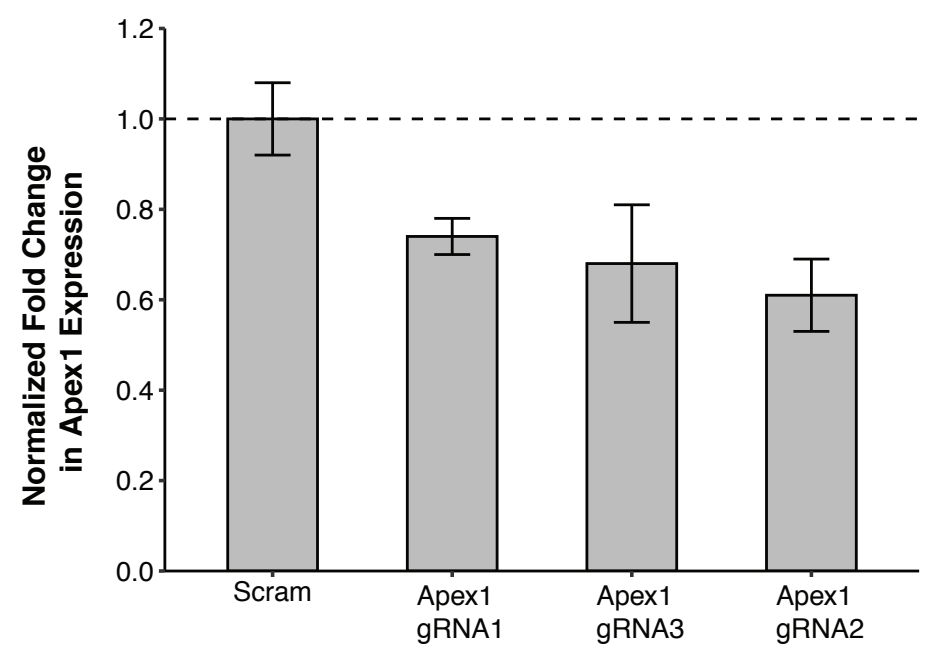

B

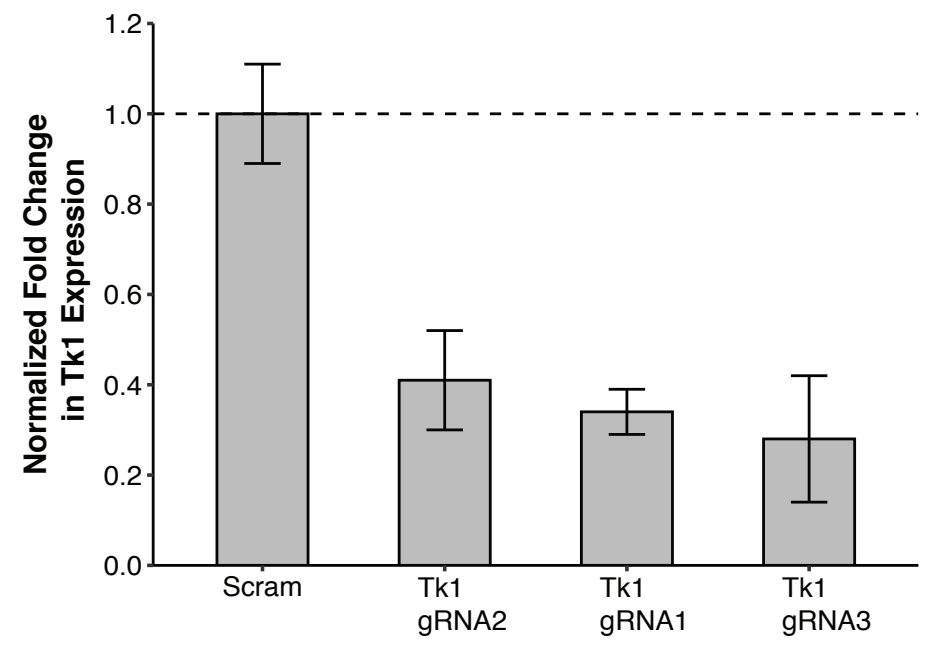

Figure S16: Validation of CRISPRi knockdown of Apex1 and Tk1 via qPCR measurements. $\Delta \Delta C_{t}$ method was used with the empty-vector cell population as the control. Levels of Apex 1 and Tk1 repression are relative to the non-targeting (scrambled) population. Data represent mean and SD of two biological replicates. 


\section{Supplemental Figure 17}

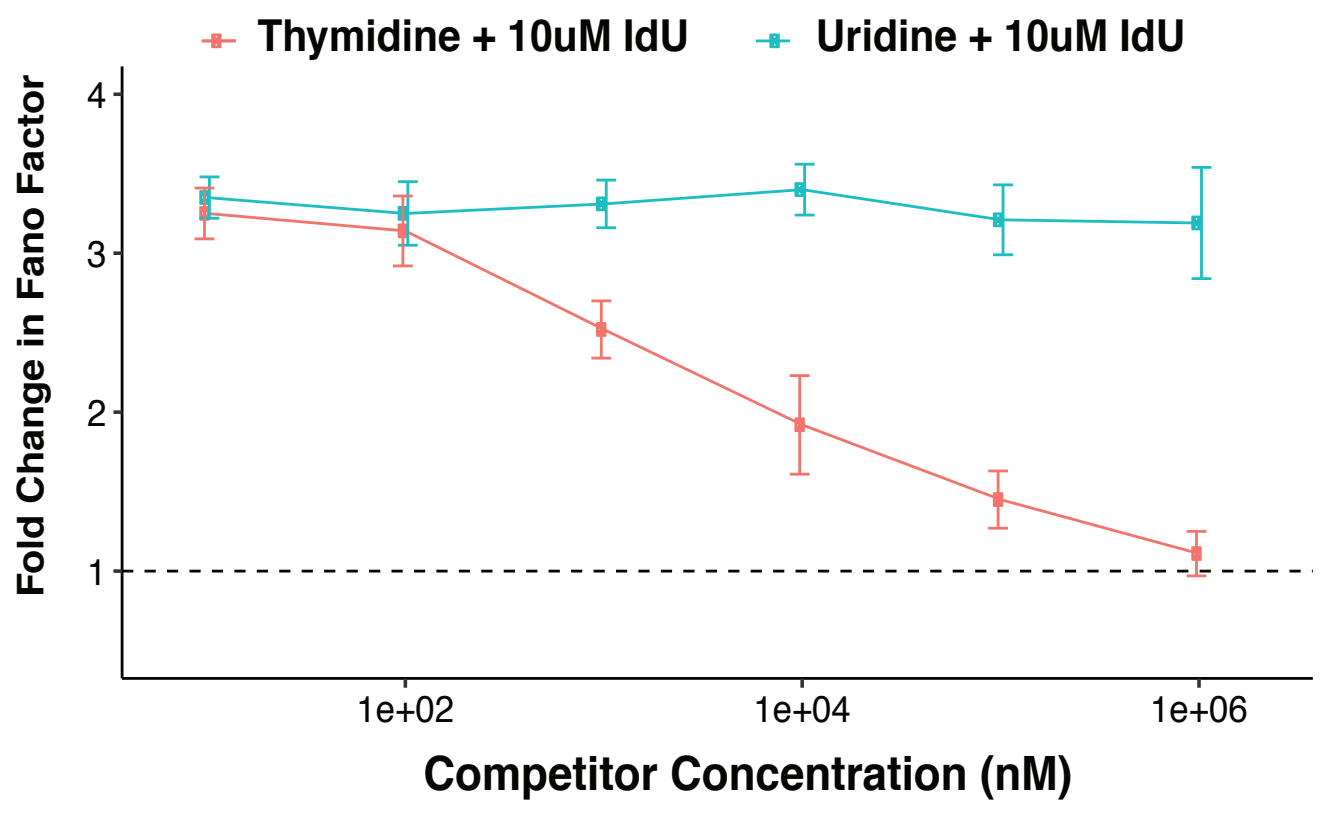

Figure S17: Thymidine competition ablates Nanog noise-enhancement from IdU.

The Fano factor of Nanog for each concentration combination is normalized to DMSO control. For all treatment combinations, IdU concentration is held constant at $10 \mu \mathrm{M}$. Concentration of thymidine (red) and uridine (blue) is reported on the $\mathrm{x}$-axis. Combination of $100 \mu \mathrm{M}$ thymidine and 10 $\mu \mathrm{M}$ IdU returns Nanog Fano factor to baseline level (DMSO control). Uridine, which is not a substrate of Tk1, fails to ablate IdU-induced noise-enhancement. Data points represent mean and SD of three biological replicates. 


\section{Supplemental Figure 18}

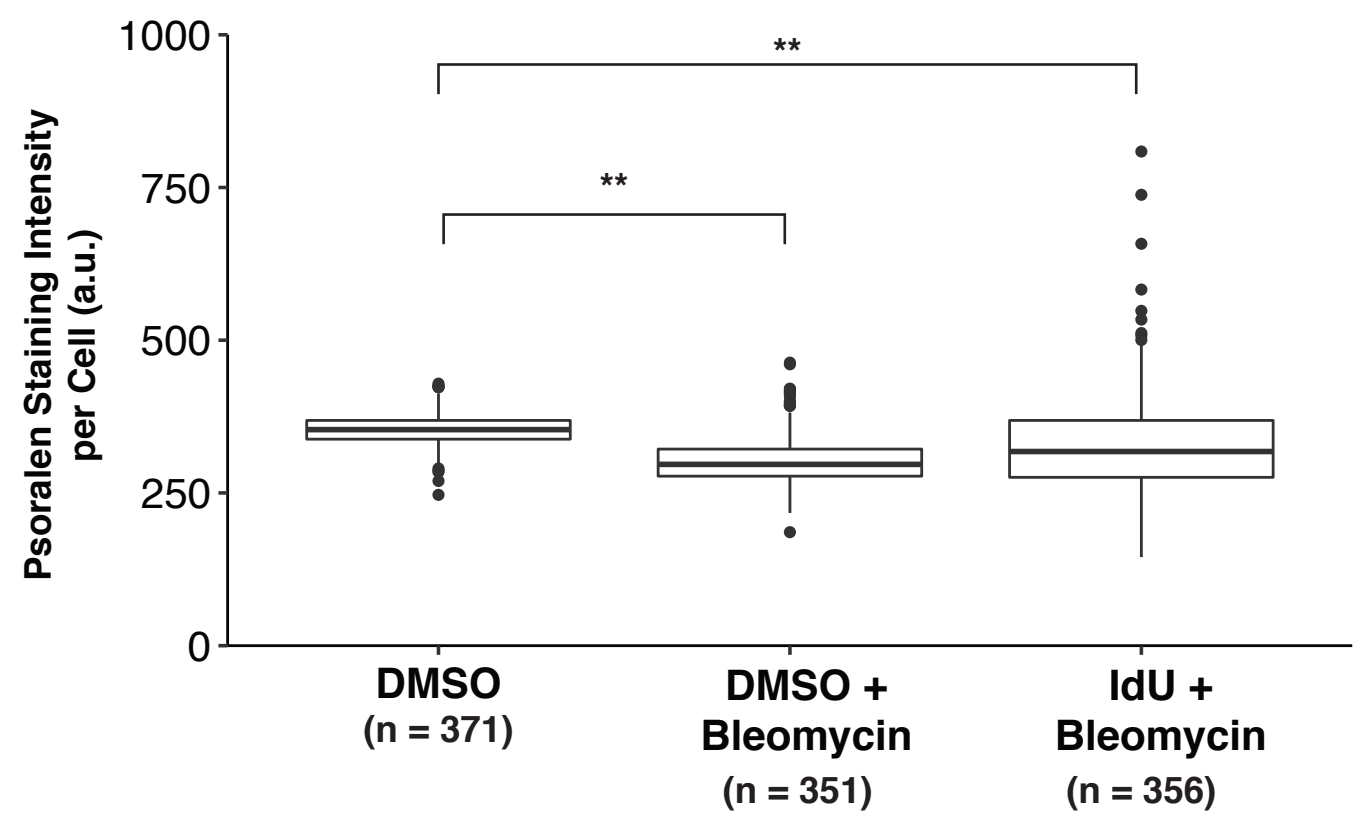

Figure S18: Bleomycin treatment reduces bTMP intercalation into DNA, validating assay sensitivity for negative supercoiling levels.

Boxplots show median \pm interquartile range of single-cell bTMP staining intensities. Treatment of mESCs with $100 \mu \mathrm{M}$ bleomycin was performed for 1 hour just prior to bTMP incubation. Bleomycin reduces the mean bTMP staining intensity for cells treated with DMSO or 10uM IdU as compared to DMSO control with no bleomycin treatment $(* * p<0.0001)$. The reduction in bTMP staining when IdU is coupled with bleomycin indicates that IdU alone in uncoiled DNA does not increase bTMP intercalation. Data shown are pooled from two biological replicates. P values were calculated using Kruskal-Wallis test followed by Tukey's multiple comparison test. 


\section{Supplemental Figure 19}

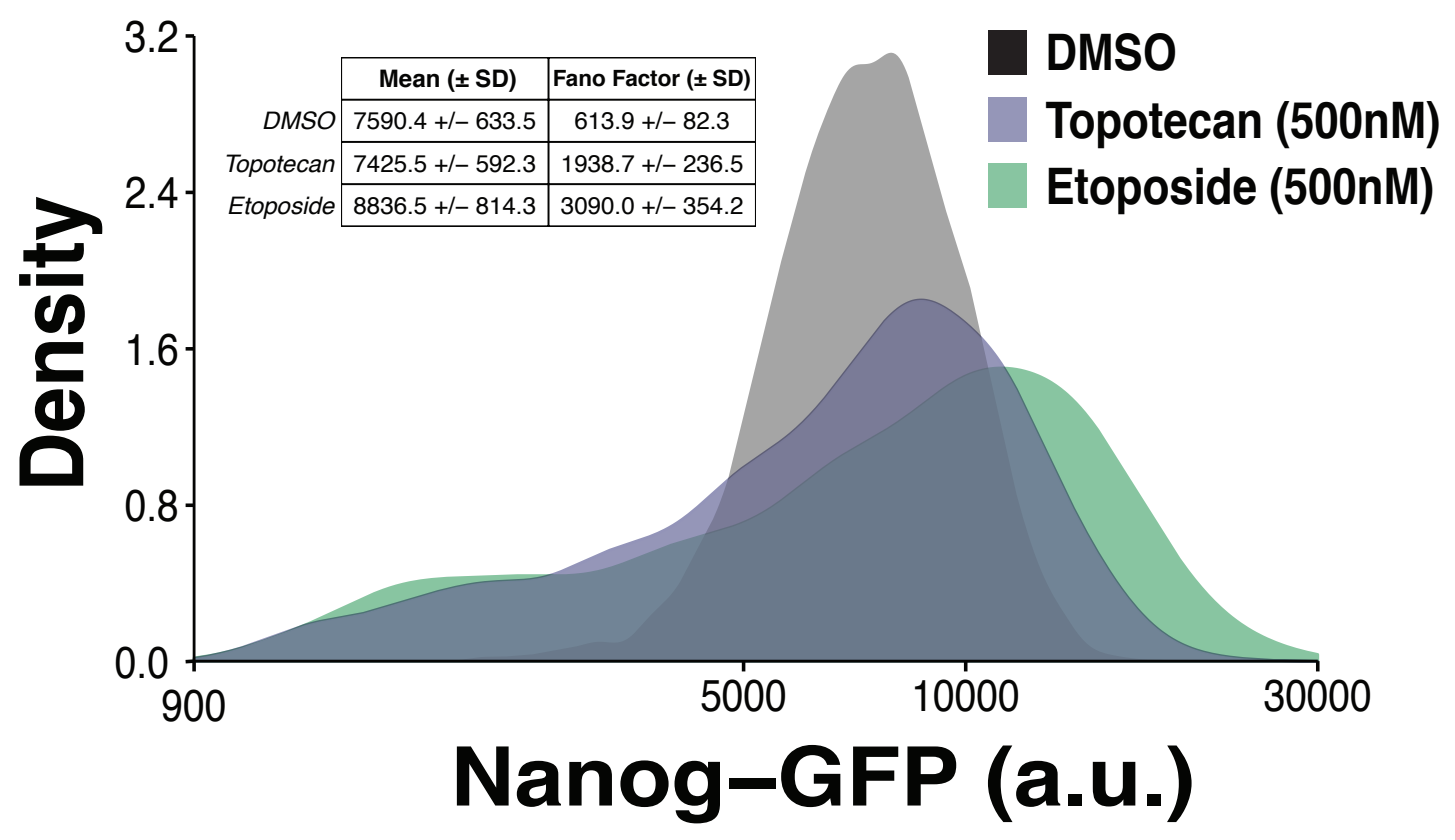

Figure S19: Small-molecule inhibition of Topoisomerase I and II increases Nanog expression variability.

Representative flow cytometry distributions of Nanog-GFP expression in mESCs treated with DMSO, 500nM topotecan or 500nM etoposide for 24 hours in 2i/Lif media. Extrinsic noise filtering via cell-size gating was performed prior to calculation of Nanog Fano factor. Table inset shows mean and Fano factor $( \pm \mathrm{SD})$ of Nanog expression averaged over three biological replicates of each treatment. 
bioRxiv preprint doi: https://doi.org/10.1101/2020.06.29.128439; this version posted June 30, 2020. The copyright holder for this preprint (which was not certified by peer review) is the author/funder, who has granted bioRxiv a license to display the preprint in perpetuity. It is made available under aCC-BY-NC-ND 4.0 International license.

\section{Supplementary Figure 20}

A

Model 1

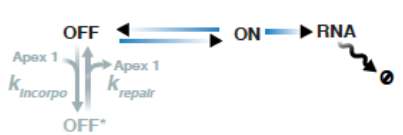

Model 2

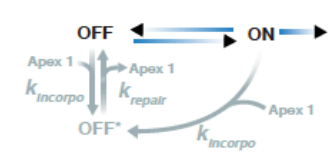

Model 3

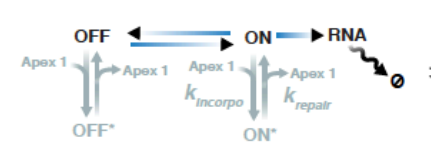

Model 4

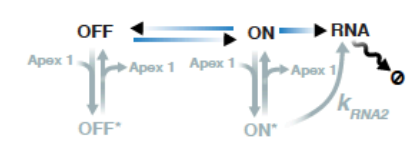

Model 5:

Transcription-

Coupled Repair

\section{Log-Likelihood Estimate}
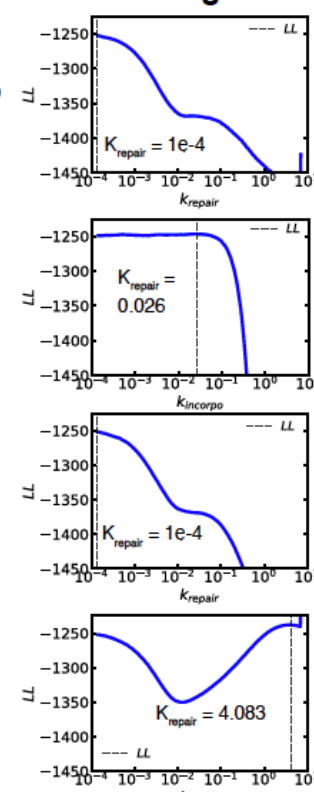

$k_{\text {repair }}$

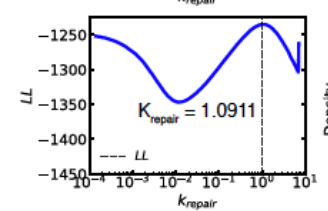

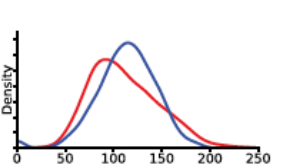
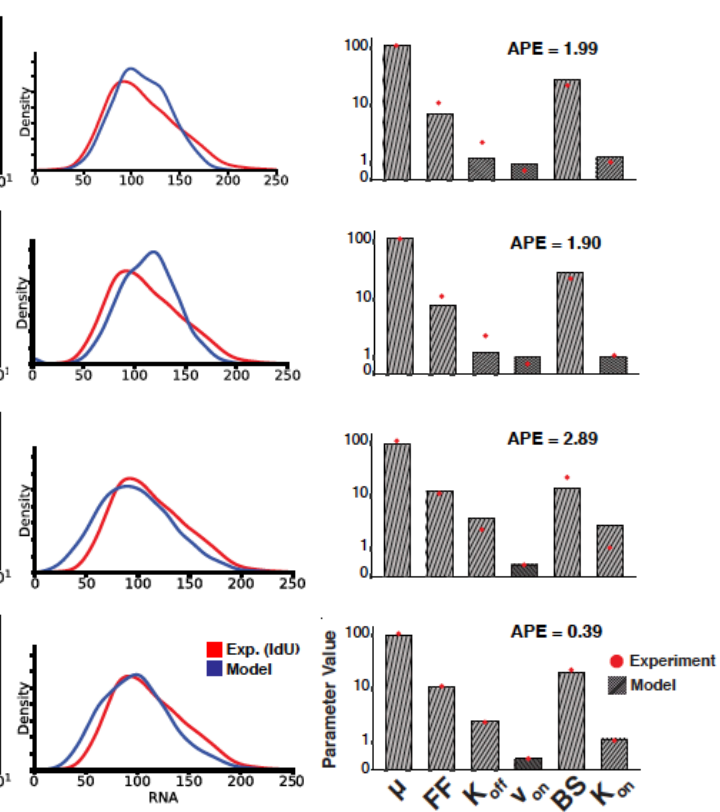

B

\begin{tabular}{cccc}
\hline Model & $\max (\mathrm{LL})$ & $\Delta_{\mathrm{i}} \mathrm{AIC}$ & $\mathrm{w}_{\mathrm{i}}$ \\
\hline Control & -1247 & 24 & 0.000 \\
1 & -1253 & 36 & 0.000 \\
2 & -1247 & 24 & 0.000 \\
3 & -1252 & 34 & 0.000 \\
4 & -1238 & 6 & 0.05 \\
5 & -1235 & 0 & 0.95 \\
\hline
\end{tabular}

C

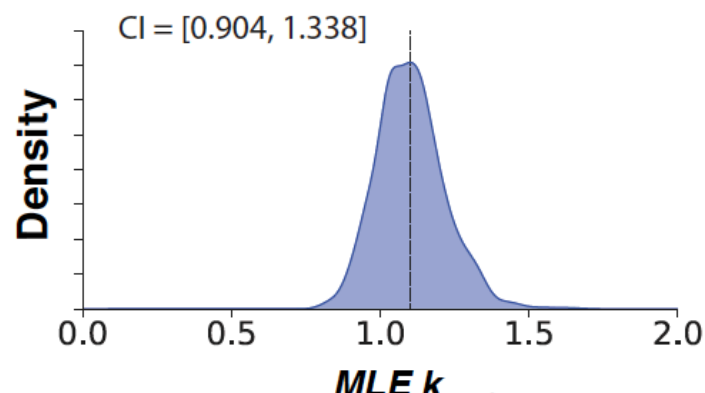


Figure S20: MLE-based approach for model selection reveals transcription-coupled repair mechanism best recapitulates experimental data.

(A) (First Column) Schematic of simulated models incorporating Apex1 into standard 2-state model of transcription. (Second Column) For each model, 500 logarithmically-spaced values of $k_{\text {repair }} \in\left[10^{-4}, 10\right]$ were simulated. For each simulated value of $k_{\text {repair }}, \log$-likelihood is calculated as described in supplementary text 5.1.2 and plotted. Dashed vertical line in each plot denotes value of $k_{\text {repair }}$ that maximizes log-likelihood estimate. (Third Column) Comparison of experimental Nanog mRNA distribution (red) to simulated distributions of Nanog mRNA (blue) for each model using value of $k_{\text {repair }}$ that maximizes log-likelihood. (Fourth Column) Macroscopic behavior of simulation results (using value of $k_{\text {repair }}$ that maximizes log-likelihood estimate) are compared to experimental data (supplementary text 5.2). Bars represent simulation values of Nanog gene expression system while red points with vertical line represent experimental data on Nanog expression from smRNA-FISH of mESCs treated with $10 \mu \mathrm{M}$ IdU. (B) For each tested model, the maximum log-likelihood value is listed along with the associated $\Delta_{i} A I C$. Model 5 (transcriptioncoupled repair) best describes experimental data based on these metrics. (C) Distribution and confidence interval (CI) of inferred $k_{\text {repair }}$ values (based on MLE) for Model 5 using bootstrapping method in which the empirical distribution of Nanog mRNA counts from smRNA-FISH data was re-sampled 1000 times with replacement (supplementary text 5.1.4). Bootstrapping results show a well peaked distribution indicating practical parameter identifiability for $k_{\text {repair }}$. 
bioRxiv preprint doi: https://doi.org/10.1101/2020.06.29.128439; this version posted June 30, 2020. The copyright holder for this preprint (which was not certified by peer review) is the author/funder, who has granted bioRxiv a license to display the preprint in perpetuity. It is made available under aCC-BY-NC-ND 4.0 International license.

\section{Supplemental Figure 21}

A

\section{Model 1}

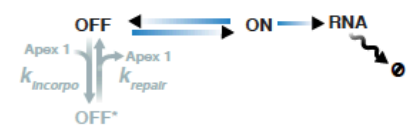

Model 2

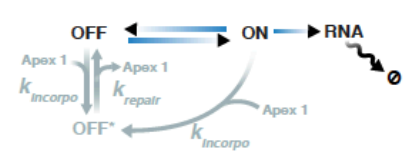

Model 3

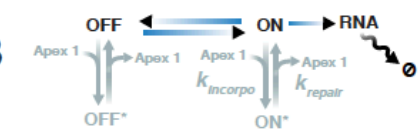

Model 4

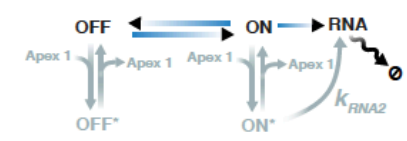

Model 5:

Transcription-

Coupled Repair
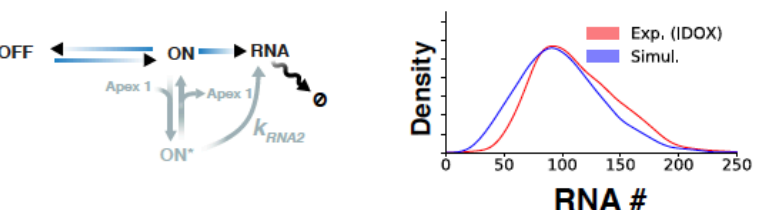

Macroscopic Behavior
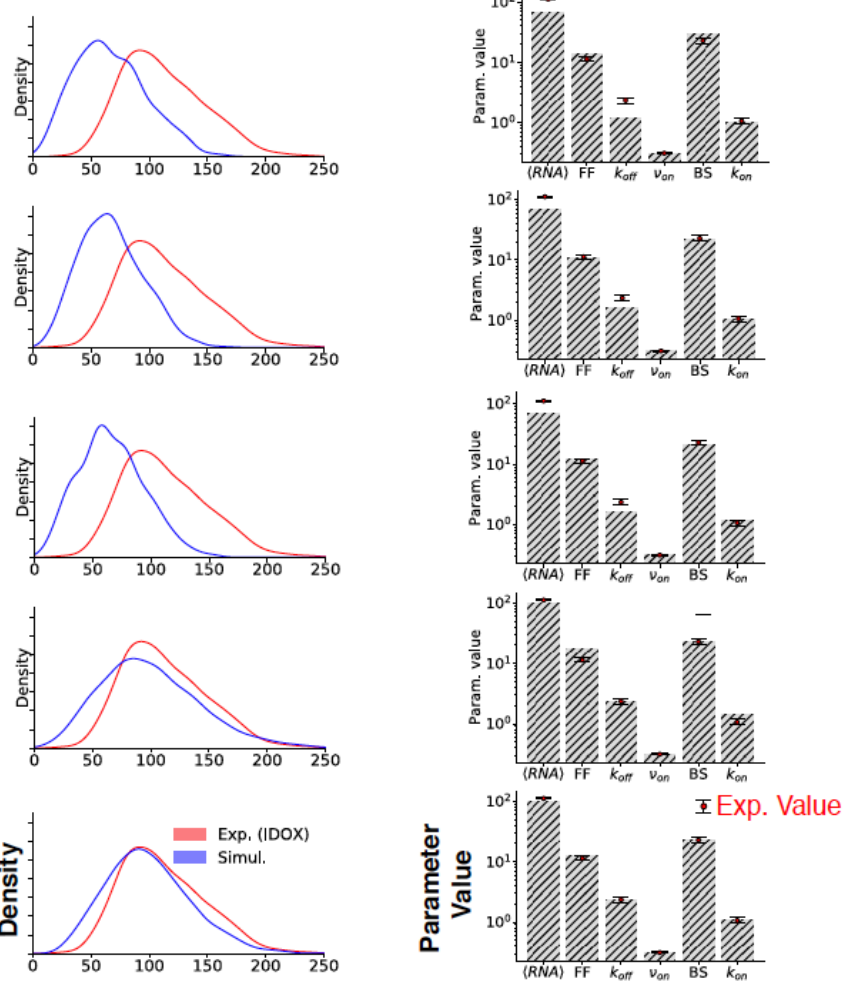
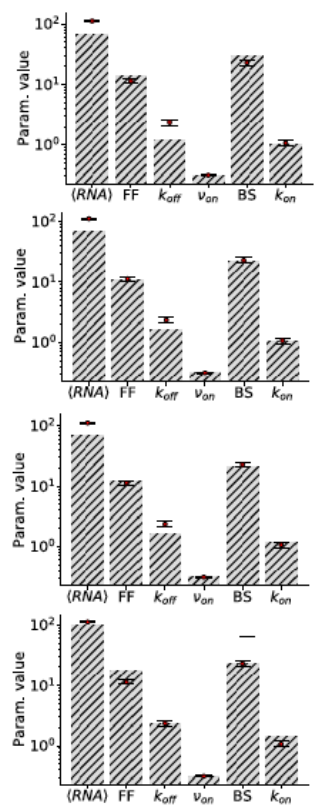

B

\begin{tabular}{cccc}
\hline Model & APE & $\hat{\mathrm{k}}_{\text {repair,APE }} \log (\mathrm{L})$ \\
\hline 1 & 1.43 & 3.27 & -1650.7 \\
2 & 0.89 & 0.43 & -1766.7 \\
3 & 0.92 & 0.74 & -1752.5 \\
4 & 0.99 & 1.71 & -1315.5 \\
5 & 0.27 & 0.89 & -1256.3 \\
\hline
\end{tabular}


Figure S21: APE-based approach for model selection concurs with MLE-based approach, identifying TCR model as best match to experimental data.

(A) (First Column) Schematic of simulated models incorporating Apex1 into standard 2-state model of transcription. (Second Column) Comparison of experimental Nanog mRNA distribution (red) to simulated distributions of Nanog mRNA (blue) for each model using value of $k_{\text {repair }}$ that minimizes absolute percentage error. (Third Column) Macroscopic behavior of simulation results (using value of $k_{\text {repair }}$ that minimizes absolute percentage error) are compared to experimental data (supplementary text 5.2). Bars represent simulation values of Nanog gene expression system while red points with vertical line represent experimental data on Nanog expression from smRNA-FISH of mESCs treated with 10 MM IdU. (B) Values of $k_{\text {repair }}$ that minimize the absolute percentage error for each model are listed. Model 5 (TCR model) yields the smallest APE and the largest log-likelihood. 


\section{Supplemental Figure 22}

A

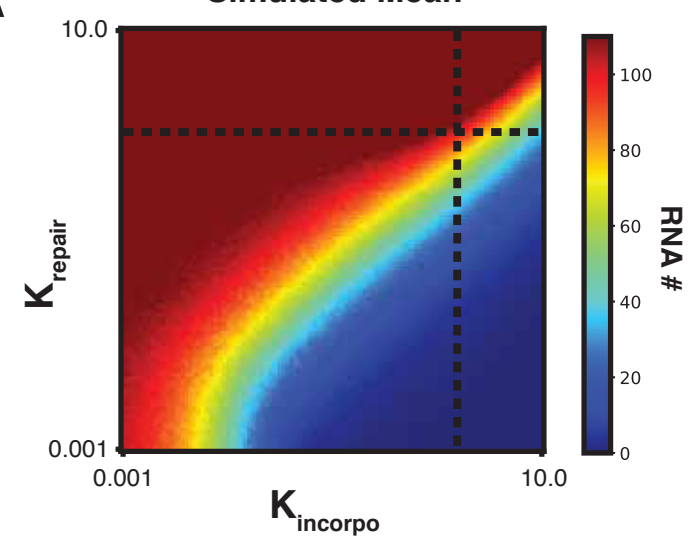

B
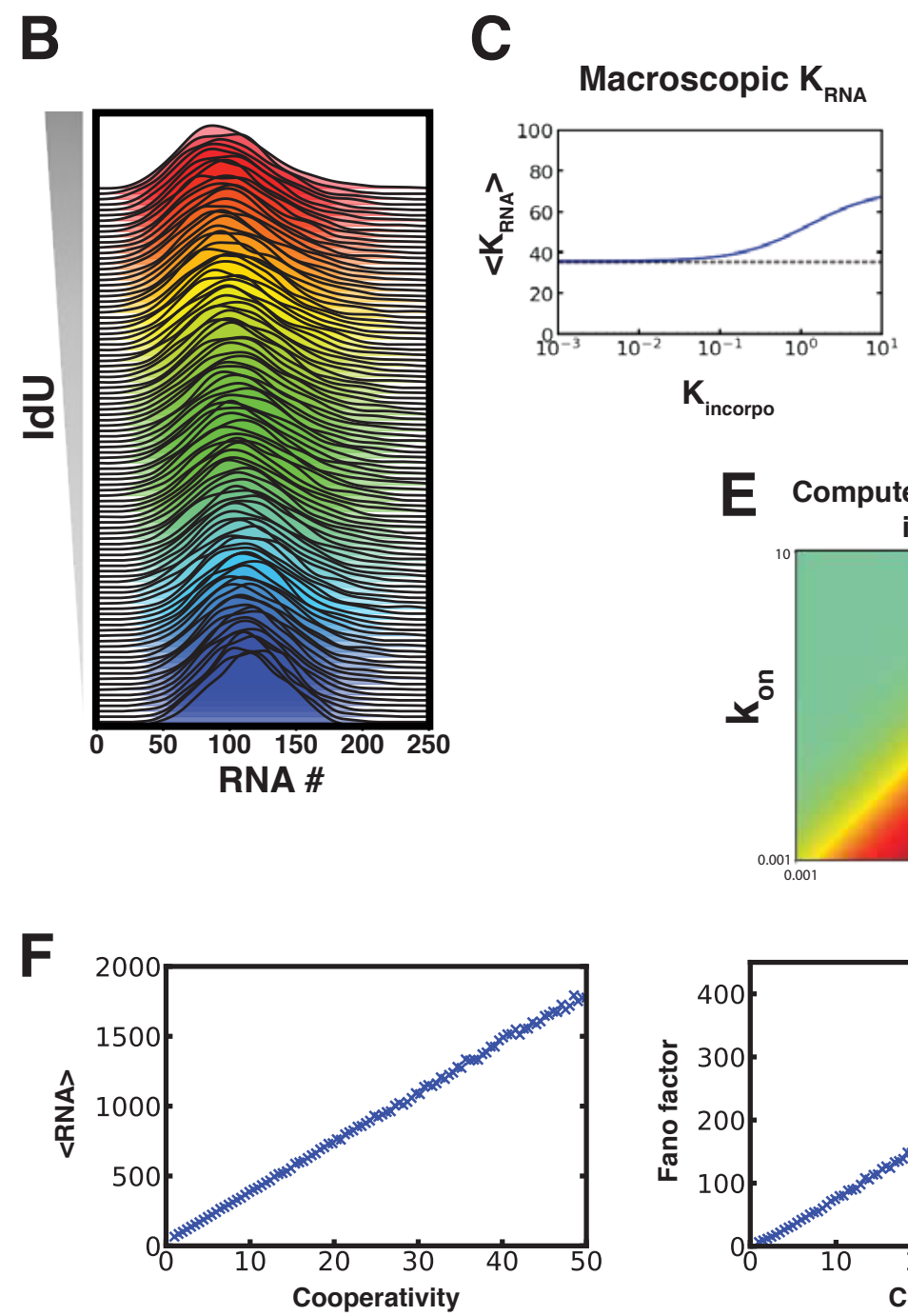
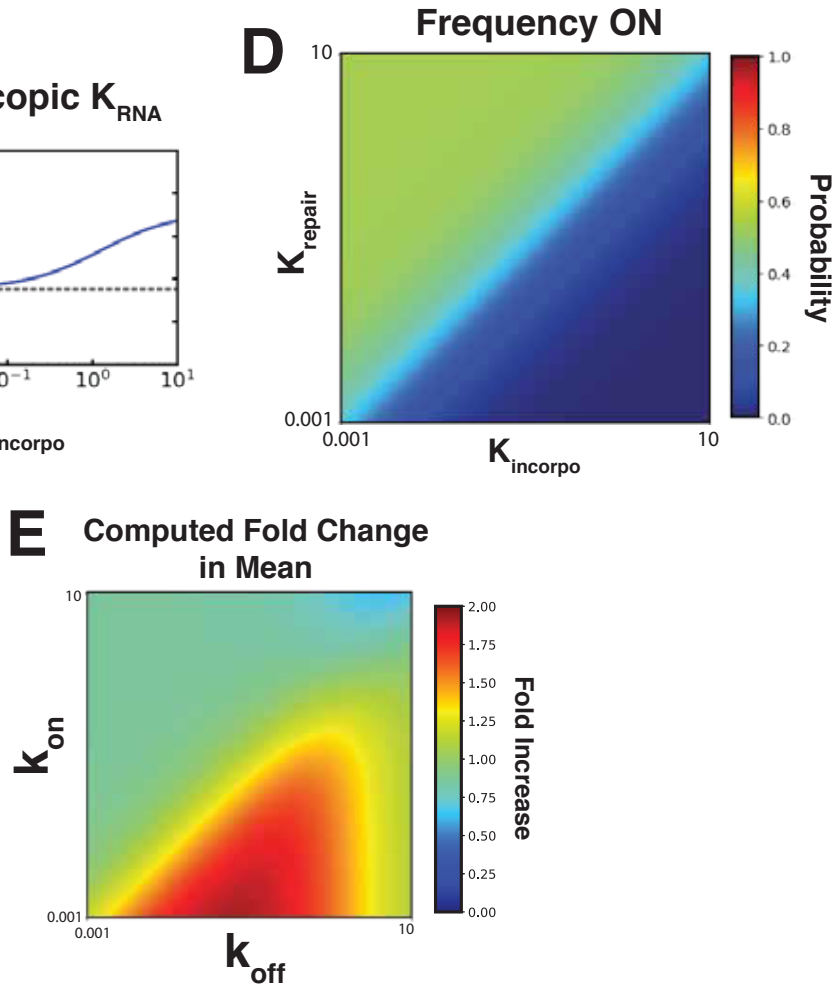

\section{Simulated Fano Factor}

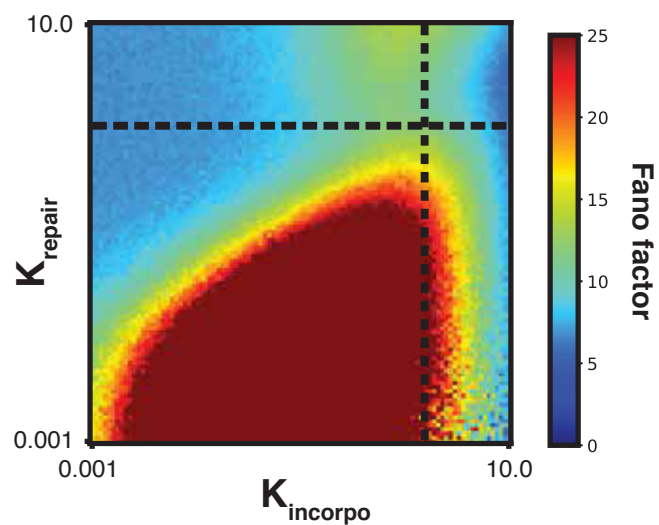

Fold Change

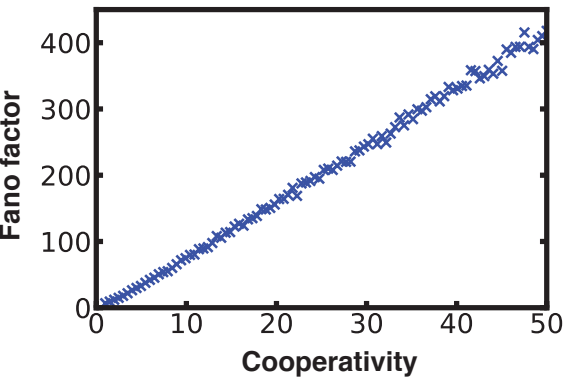


Figure S22: Sensitivity analysis of model parameters reveals phase-space for modulation of Nanog variability independently of mean.

(A) Heatmaps displaying mean (left) and Fano factor (right) of Nanog mRNA from simulation results of TCR model as a function of $k_{\text {repair }}$ and $k_{\text {incorpo }}$ values spanning four orders of magnitude (supplementary text 6.1). Dashed horizontal and vertical lines represent inferred values of $k_{\text {repair }}$ and $k_{\text {incorpo }}$ that match experimental Nanog gene expression system in the presence of $10 \mu \mathrm{M}$ IdU. Multiple regions of the parameter phase-space exhibit constant mean output with unique levels of variability (Fano factor) demonstrating how mean and variability are tuned independently. (B) Simulated distributions of Nanog mRNA with increasing concentration of IdU which increases $k_{\text {incorpo }}$ Simulation results demonstrate how TCR model allows for maintenance of mean output with increasing variability as concentration of IdU is increased. (C) Effective transcription rate of Nanog gene expression system as a function of $k_{\text {incorpo }}$. As IdU incorporation and subsequent Apex 1 recruitment increases, the effective transcription rate increases as well. This represents the compensatory mechanism of model 5 allowing for maintenance of mean output with increasing incorporation of IdU. (D) Heatmap displaying fraction of time that the Nanog gene expression is in the macroscopic ON state as a function of $k_{\text {repair }}$ and $k_{\text {incorpo }}$ values. (E) Heatmap displaying fold change in mean as a function of microscopic $k_{\text {off }}$ and $k_{\text {on }}$ values (supplementary text 6.2). Fold change is calculated as the output of Model 5 relative to model 0 (canonical 2-state model) for the same set of $k_{o f f}$ and $k_{o n}$ values. For a gene whose $k_{o f f}>>k_{o n}$, addition of IdU to the system increases the mean output. (F) Mean mRNA and Fano factor of Model 5 output as a function of the cooperativity term which describes how strongly the transcription rate is amplified following completion of repair (supplementary text 6.3). 


\section{Supplementary Figure 23}

A

\section{BrdU}
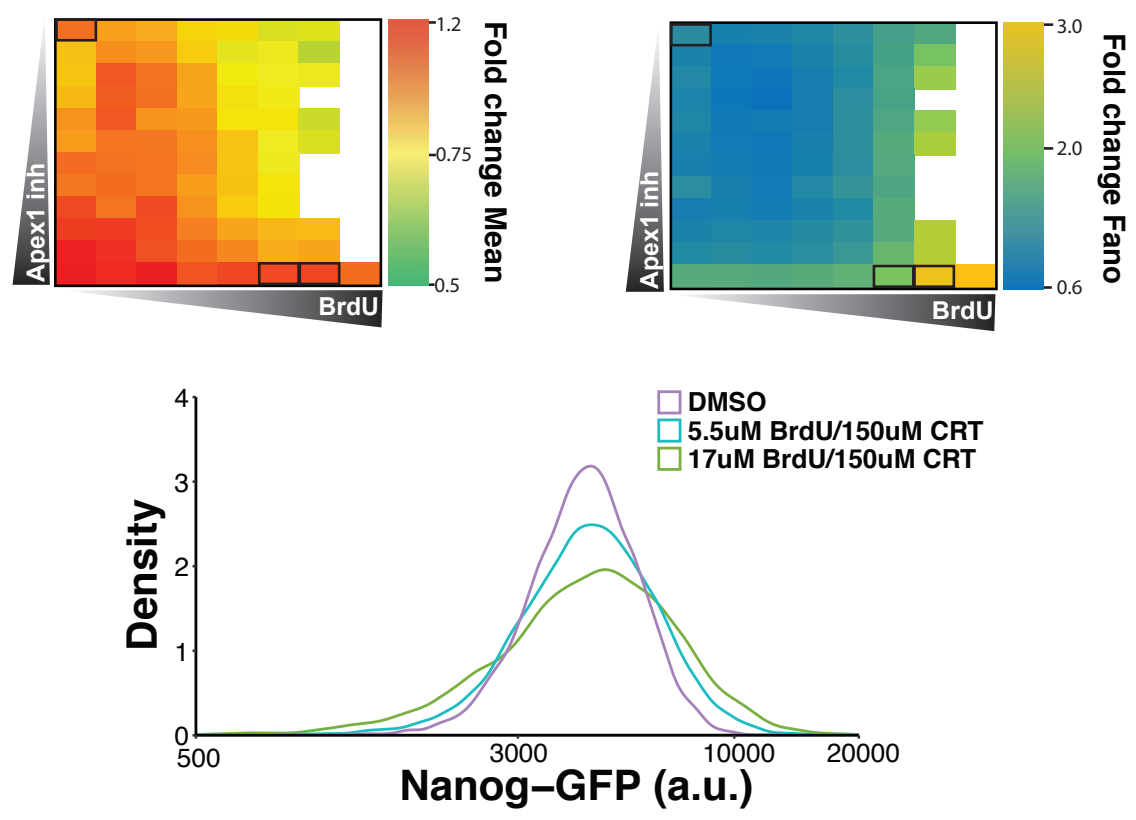

B

$\mathrm{HmU}$
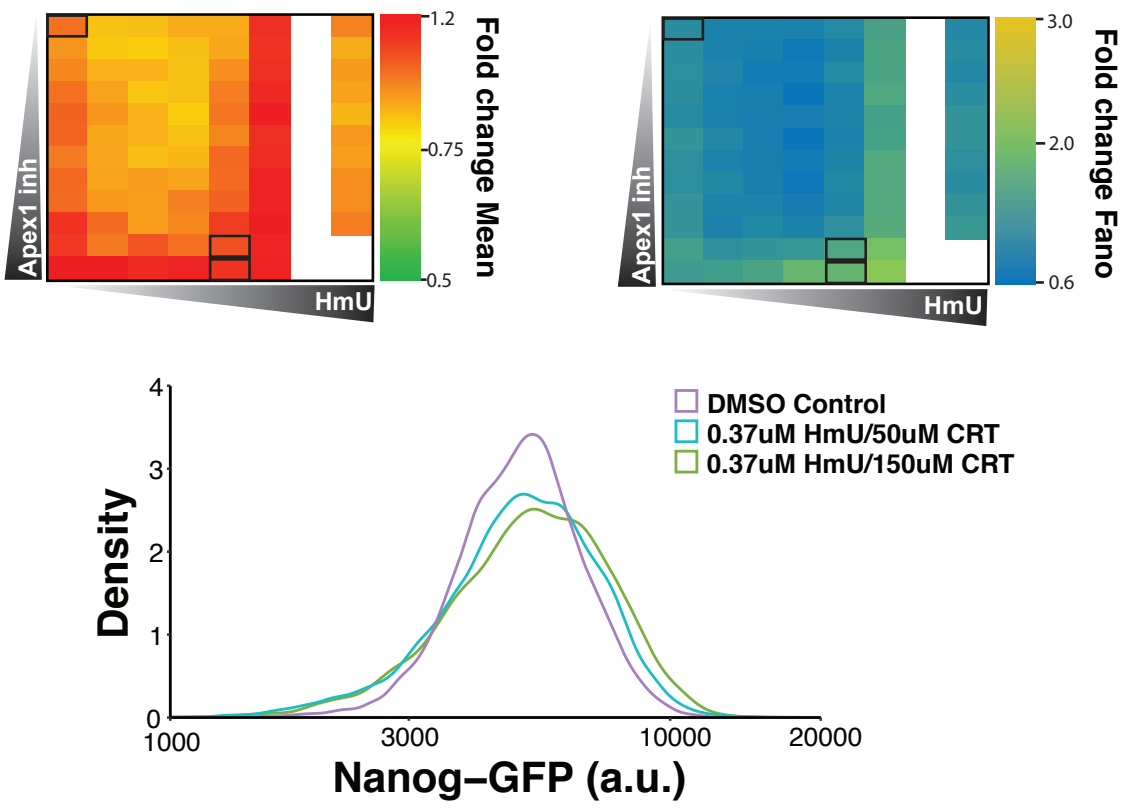
Figure S23: Treatment with BrdU or HmU in combination with CRT0044876 allows for tuning of Nanog variability independently of the mean.

(A) Testing of 96 concentration combinations of BrdU and CRT0044876 (apex1 endonuclease domain inhibitor) to validate tunability of Nanog variability. BrdU and CRT0044876 were used to increase binding and decrease unbinding of Apex1 respectively. Nanog-GFP mESCs grown in 96-well plates were treated with 12 concentrations of CRT0044876 ranging from 0 to $150 \mathrm{uM}$ in combination with 8 concentrations of BrdU ranging from 0 to $50 \mathrm{uM}$. Data represent average of two biological replicates. (Top left and top right panels) 96-well heatmaps displaying fold change in Nanog mean and Fano factor for each drug combination as compared to DMSO (topleftmost well). Insufficient number of cells $(<50,000)$ for extrinsic noise filtering were recorded from white wells. (Bottom Panel) Representative flow cytometry distributions from highlighted wells (black rectangles). Nanog variability increases independently of the mean. (B) Testing of 96 concentration combinations of HmU and CRT0044876 (apex1 endonuclease domain inhibitor) to validate tunability of Nanog variability. $\mathrm{HmU}$ is a naturally found, Tet-induced oxidation product of thymine. Nanog-GFP mESCs grown in 96-well plates were treated with 12 concentrations of CRT0044876 ranging from 0 to $150 \mathrm{uM}$ in combination with 8 concentrations of $\mathrm{HmU}$ ranging from 0 to $10 \mathrm{uM}$. Data represent average of two biological replicates. (Top left and top right panels) 96-well heatmaps displaying fold change in Nanog mean and Fano factor for each drug combination as compared to DMSO (top-leftmost well). Insufficient number of cells $(; 50,000)$ for extrinsic noise filtering were recorded from white wells. (Bottom Panel) Representative flow cytometry distributions from highlighted wells (black rectangles). As with IdU and BrdU, Nanog variability increases independently of the mean. 


\section{Supplemental Figure 24}

\section{A Poisson- $\beta$ Model Consistency}

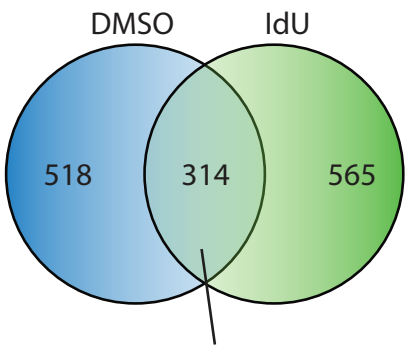

Filtered genes based on consistency between inferred kinetic rates and experimental data

B Promoter Activation

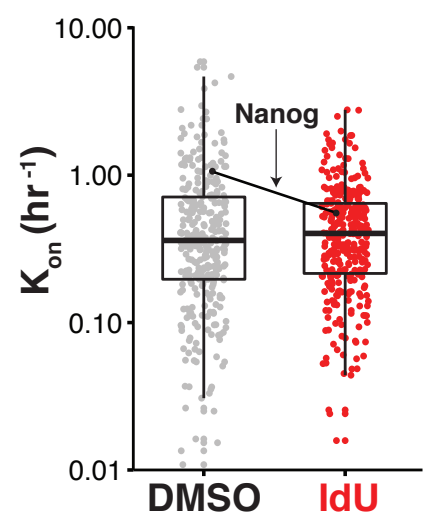

C

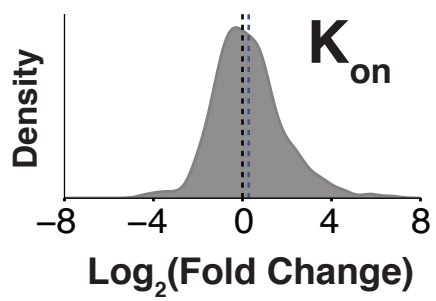

D

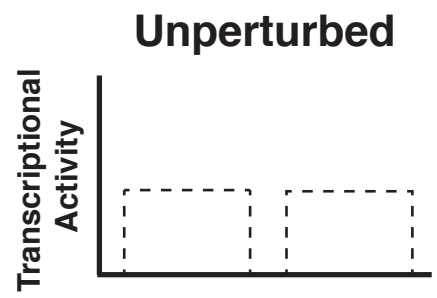

Time
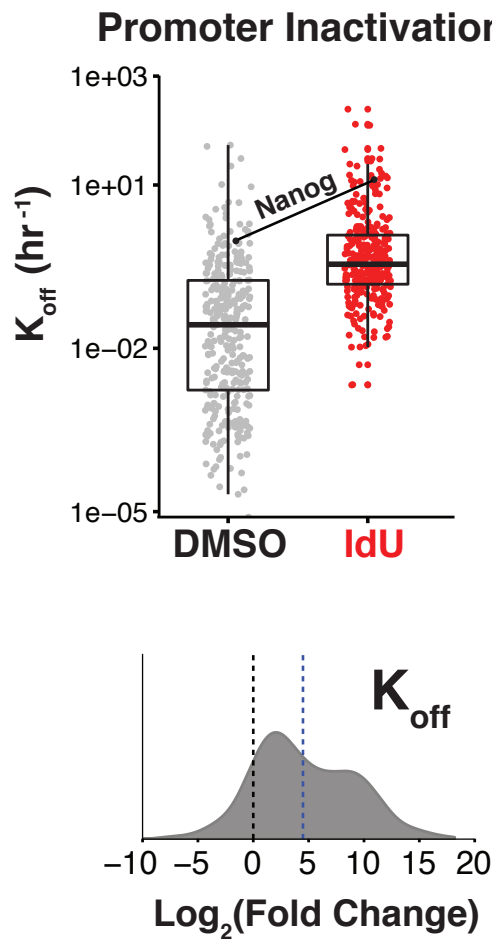

\section{Base Excision Repair}

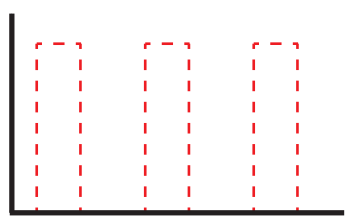

Time
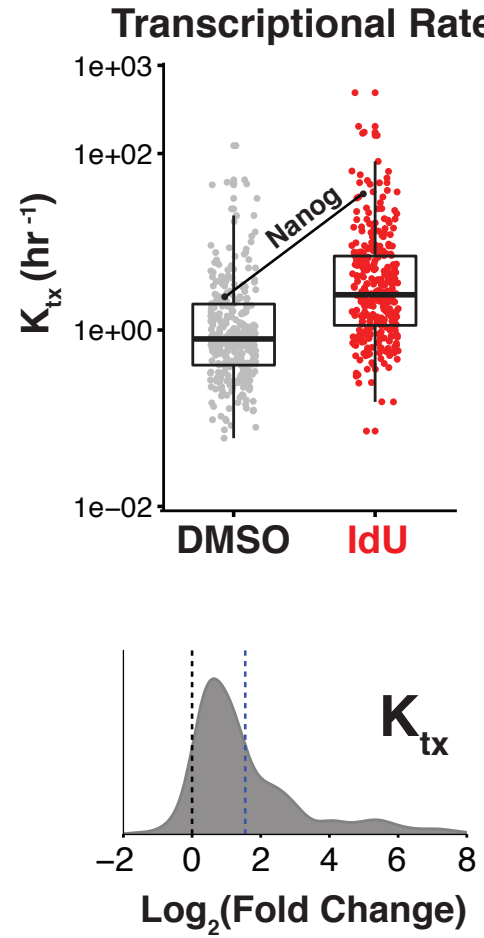
Figure S24: Highly variable genes exhibit shorter but more intense transcriptional bursts. (A) Of the 945 genes classified as highly variable with IdU treatment, we were able to estimate parameters of the 2-state model for 314 of these genes (supplementary text 7). (B) Boxplots show median \pm interquartile range of parameter estimates with each point representing a gene. (C) Distributions of fold change in bursting kinetics between IdU and DMSO conditions for 314 highly variable genes. Dashed blue line signifies mean of distribution. Majority of highly variable genes exhibit increased $K_{O F F}$ and $K_{t x}$, which is consistent with TCR model. (D) Base-excision repair orchestrates shorter but more intense transcriptional bursts to maintain mean expression for genes with diverse bursting kinetics. 


\section{Supplementary Figure 25}
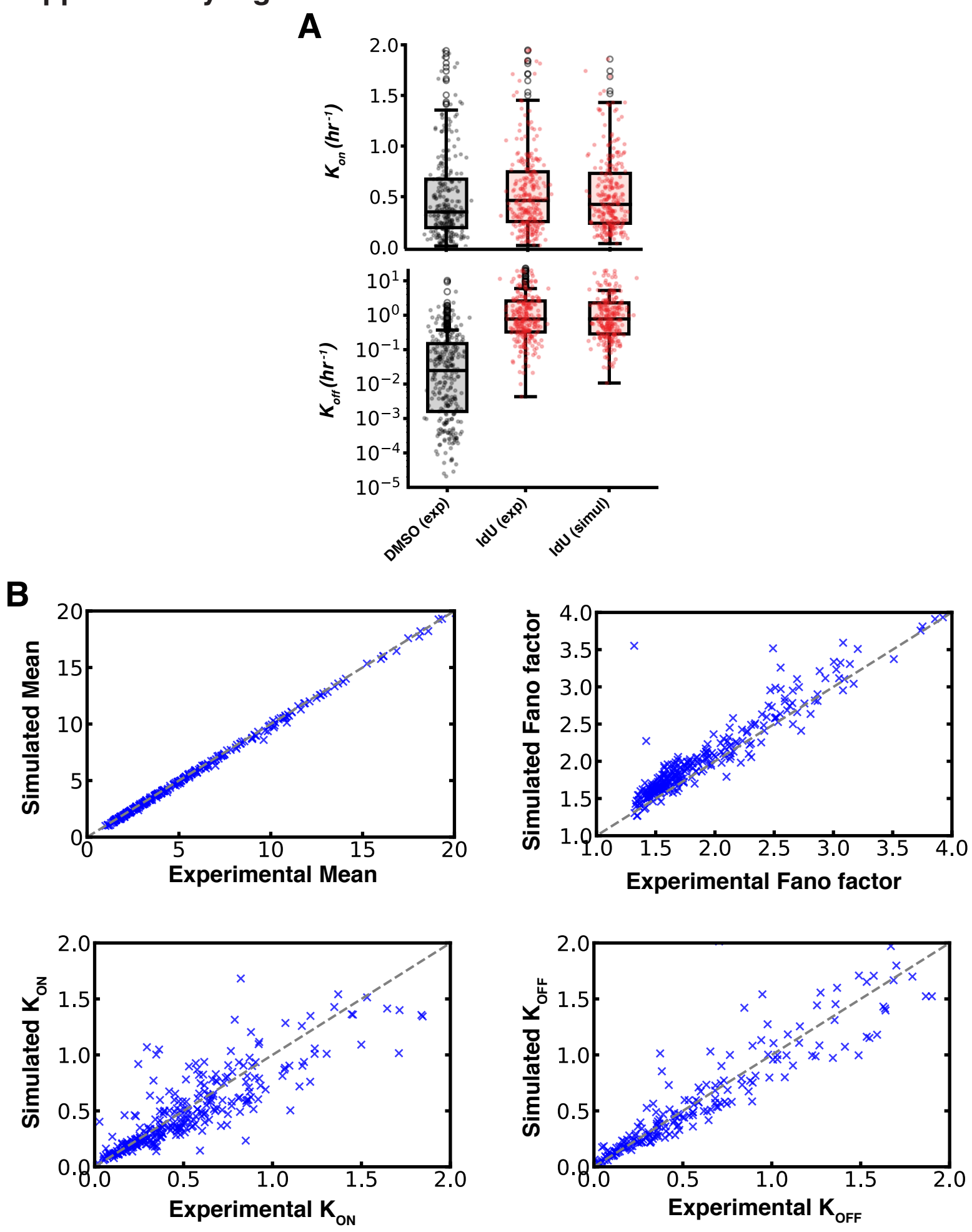
Figure S25: TCR model provides unifying mechanism for noise-enhancement of genes with different bursting kinetics.

(A) Experimental values (exp) of macroscopic $K_{O N}$ and $K_{O F F}$, as derived from a momentsmatching technique applied to scRNA-seq data, are compared to predicted values (simul) derived from simulations of TCR model. Each point represents a gene. Boxplots show median \pm interquartile range of parameter values. (B) Experimental values of mean, Fano factor, $K_{O N}$, and $K_{O F F}$ (based on scRNA-seq data) are compared to simulated values derived from TCR model. 


\section{Supplemental Figure 26}

A

\section{Reconstructed Waddington Landscape}
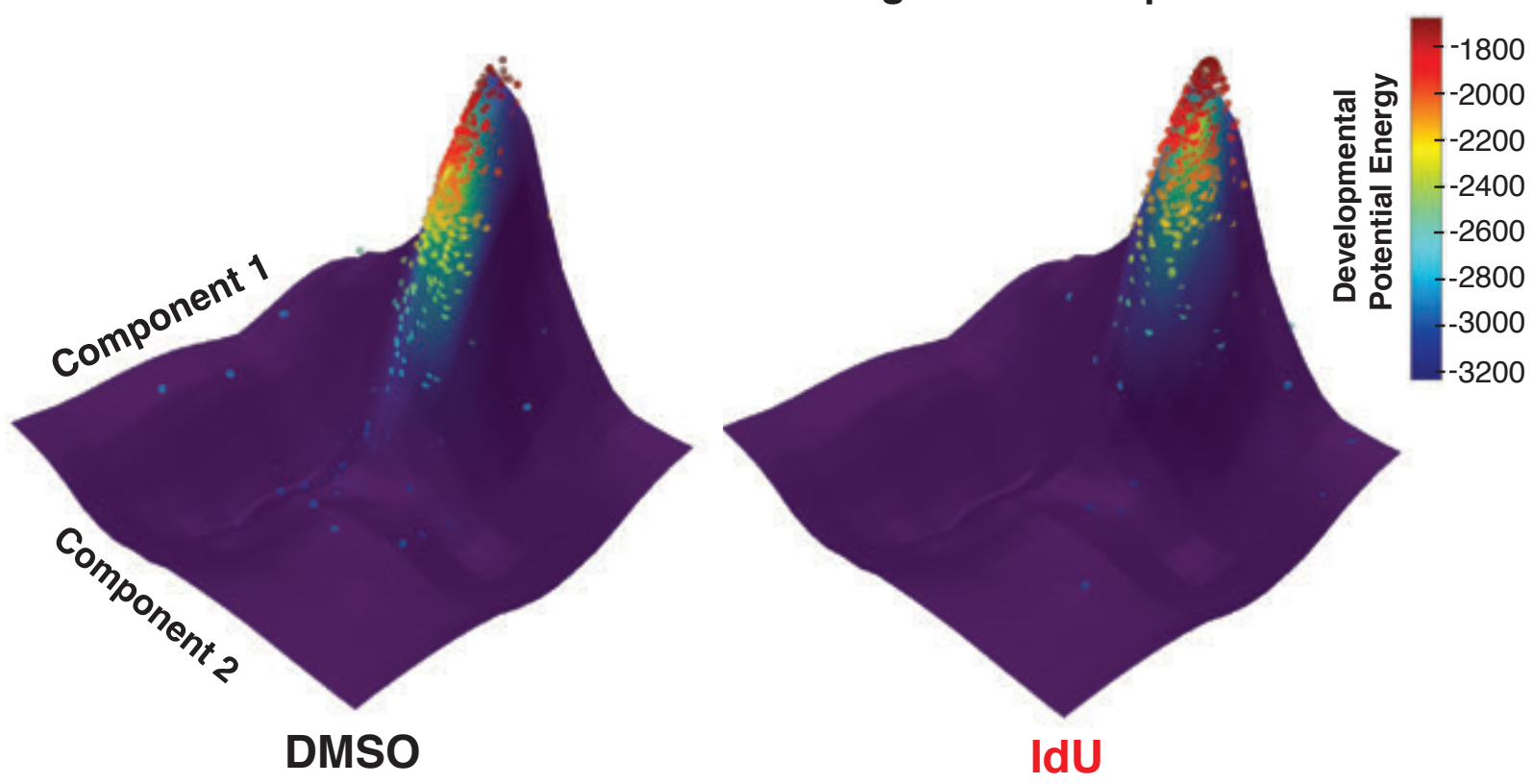

B

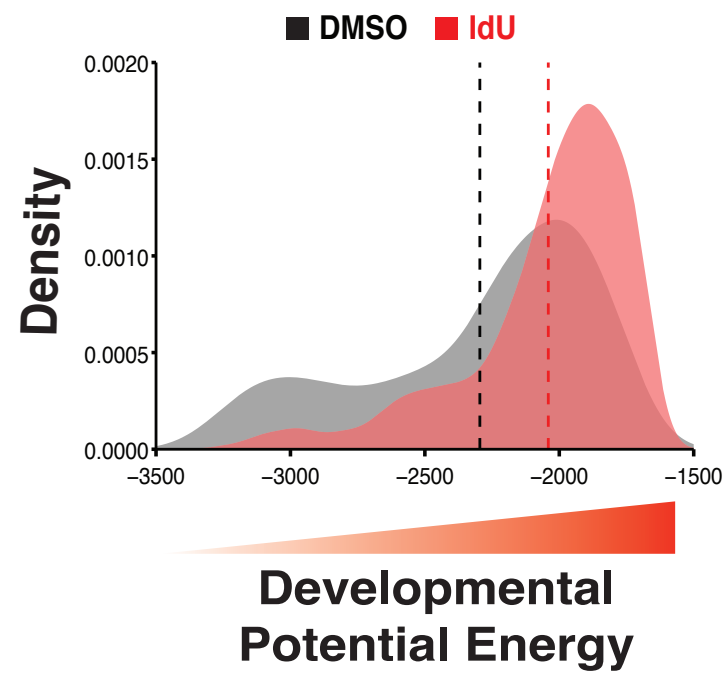


Figure S26: Amplification of transcriptional fluctuations destabilizes cellular identity resulting in greater cellular plasticity.

(A) Reconstruction of Waddington's landscape using scRNA-seq data of mESCs treated with DMSO (left) or IdU (right). Each point represents a cell. A Gaussian process latent variable model (GP-LVM) was used for dimensionality reduction to create a 2-D map of cell clustering, represented by component 1 (y-axis) and component 2 (x-axis). The z-axis represents the calculated potential energy (distance from an attractor) of a cell's gene expression state with lower values indicating greater proximity to an attractor and thus lower developmental potential. Cells are colored according to their height (developmental potential energy, z-axis value) on the landscape as denoted by the associated color bar. Underlying shading of landscape represents density of points with purple being the least dense and yellow being the most dense. (B) Distributions of developmental potential energy (z-axis values from Waddington landscape in panel A) for mESCs treated with DMSO or $10 \mu \mathrm{M}$ IdU for 24 hours. Dashed vertical lines signify the mean of each distribution, with IdU-treated cells demonstrating greater potential energies. 


\section{Supplemental Figure 27}

A

Noise Enhancement in

Mouse Embryonic Fibroblasts
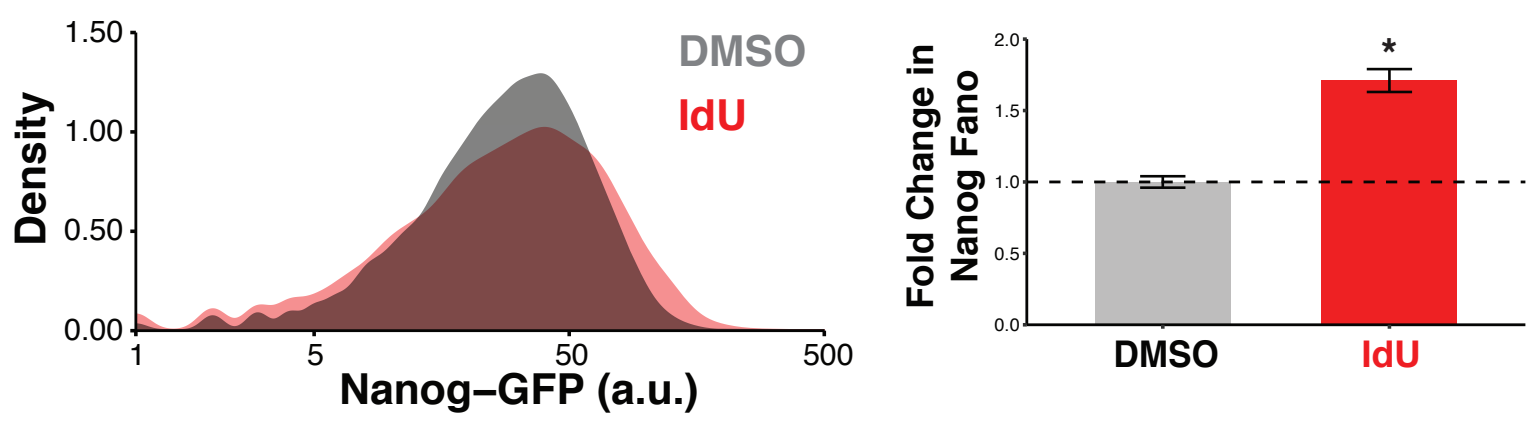

B
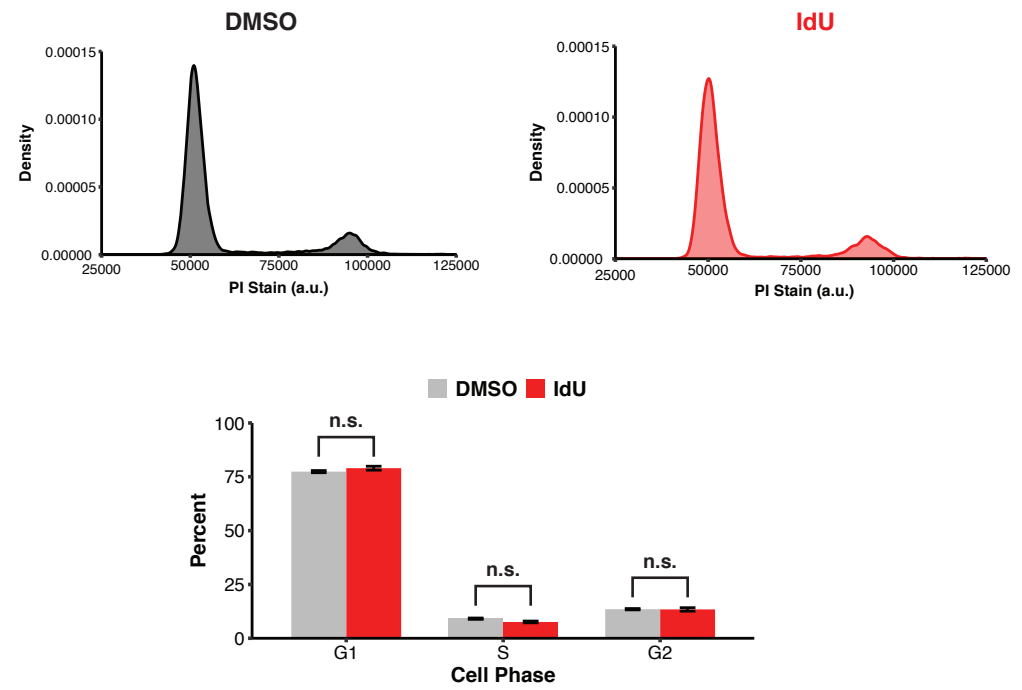

C

Day 2 of MEF Reprogramming

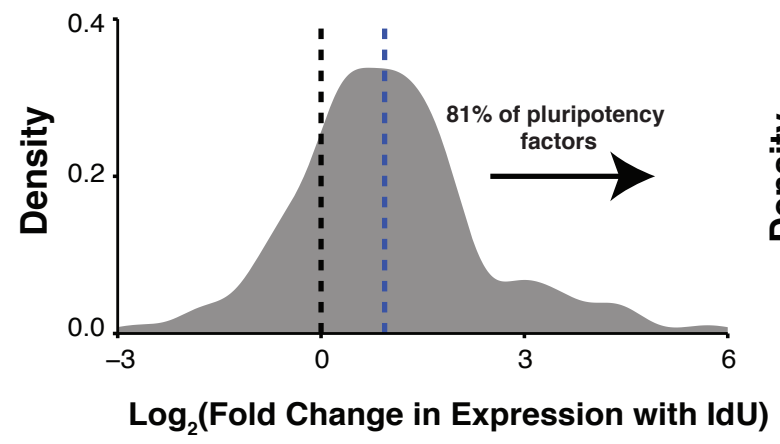

Day 5 of MEF Reprogramming

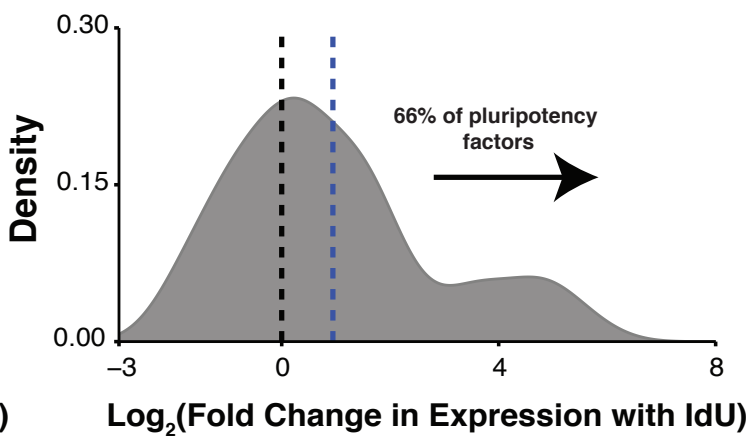


Figure S27: IdU treatment enhances conversion of mouse embryonic fibroblasts (MEFs) into induced pluripotent stem cells (iPSCs).

(A) Secondary MEFs with the endogenous Nanog locus tagged with GFP were treated with $4 \mu \mathrm{M}$ IdU or equivalent volume DMSO for 48 hours in MEF media. (Right) Representative flow cytometry distributions of Nanog-GFP expression in secondary MEFs after 48 hour treatment with IdU or DMSO. (Left) Quantification of Nanog Fano factor demonstrates that IdU treatment increases expression variability as compared to DMSO control ${ }^{*} \mathrm{p}=0.003$, by a two-tailed, unpaired Student's t test). Data represent mean and SD of three biological replicates. (B) (Top) Representative flow cytometry distributions of propidium iodide staining for Nanog-GFP secondary MEFs treated with either DMSO or $4 \mu \mathrm{M}$ IdU for 48 hours in MEF media. No signs of aneuploidy are visible, indicating Nanog expression variability is not due to cell-to-cell variability in gene copy numbers. (Bottom) Percent of cells in each phase of the cell cycle for DMSO and IdU treatments based on propidium iodide staining. IdU treatment does not alter cell-cycle progression, indicating enhanced reprogramming is not due to accelerated cellular division. Data represent mean and SD of three biological replicates. P values were calculated using a two-tailed, unpaired Student's $t$ test. (C) Bulk RNA-seq was conducted on days 2 and 5 of doxycycline-induced reprogramming of secondary MEFs supplemented with $4 \mu \mathrm{M}$ IdU or equivalent volume DMSO for the first 48 hours. Distributions of fold change in expression for 129 pluripotency genes (taken from Mouse Genome Informatics, gene ontology term: 0019827) in the IdU condition as compared to the DMSO control are shown. Dashed blue line represents mean of distribution. $81 \%$ and $66 \%$ of the pluripotency factors show increased expression with the addition of IdU as compared to DMSO control at days 2 and 5 of reprogramming, respectively. Noise amplification during early stages of reprogramming accelerates activation of pluripotency network. 


\section{Captions for supplementary tables S1-S6}

Table S1 (attached separately)

Sequences of smRNA-FISH oligonucleotide probes for first intron of Nanog and GFP.

Table S2 (attached separately)

Inferred macroscopic kinetic rates of 2-state random telegraph model for Nanog in DMSO and IdU conditions.

Table S3 (attached separately)

List of nucleoside analogs that were screened for ability to increase to Nanog protein variability.

Table S4 (attached separately)

Gene targets and sequences of gRNAs used in CRISPRi screen.

Table S5 (attached separately)

Sequences of primers used for RT-qPCR verification of Apex1 and Tk1 knockdown.

Table S6 (attached separately)

Concentrations and layout of compound plates used for testing of IdU, BrdU, or hmU in combination with CRT0044876. 